Nonlinear Processes in Geophysics, 12, 891-945, 2005

SRef-ID: $1607-7946 / \mathrm{npg} / 2005-12-891$

European Geosciences Union

(c) 2005 Author(s). This work is licensed

under a Creative Commons License.

\title{
Self-similarity of wind-driven seas
}

\author{
S. I. Badulin ${ }^{2}$, A. N. Pushkarev ${ }^{4,5}$, D. Resio ${ }^{3}$, and V. E. Zakharov ${ }^{1,2,4,5}$ \\ ${ }^{1}$ University of Arizona, Tucson, USA \\ ${ }^{2}$ P.P.Shirshov Institute of Oceanology of Russian Academy of Sciences, Moscow, Russia \\ ${ }^{3}$ Waterways Experiment Station, USA, Vicksburg, Massachusets, USA \\ ${ }^{4}$ Landau Institute for Theoretical Physics of Russian Academy of Sciences, Moscow, Russia \\ ${ }^{5}$ Waves and Solitons LLC, Phoenix, Arizona, USA
}

Received: 19 September 2005 - Revised: 11 October 2005 - Accepted: 11 October 2005 - Published: 3 November 2005

\begin{abstract}
The results of theoretical and numerical study of the Hasselmann kinetic equation for deep water waves in presence of wind input and dissipation are presented. The guideline of the study: nonlinear transfer is the dominating mechanism of wind-wave evolution. In other words, the most important features of wind-driven sea could be understood in a framework of conservative Hasselmann equation while forcing and dissipation determine parameters of a solution of the conservative equation. The conservative Hasselmann equation has a rich family of self-similar solutions for duration-limited and fetch-limited wind-wave growth. These solutions are closely related to classic stationary and homogeneous weak-turbulent Kolmogorov spectra and can be considered as non-stationary and non-homogeneous generalizations of these spectra. It is shown that experimental parameterizations of wind-wave spectra (e.g. JONSWAP spectrum) that imply self-similarity give a solid basis for comparison with theoretical predictions. In particular, the selfsimilarity analysis predicts correctly the dependence of mean wave energy and mean frequency on wave age $C_{p} / U_{10}$. This comparison is detailed in the extensive numerical study of duration-limited growth of wind waves. The study is based on algorithm suggested by Webb (1978) that was first realized as an operating code by Resio and Perrie $(1989,1991)$. This code is now updated: the new version is up to one order faster than the previous one. The new stable and reliable code makes possible to perform massive numerical simulation of the Hasselmann equation with different models of wind input and dissipation. As a result, a strong tendency of numerical solutions to self-similar behavior is shown for rather wide range of wave generation and dissipation conditions. We found very good quantitative coincidence of these solutions with available results on duration-limited growth, as well as with experimental parametrization of fetch-limited spectra JONSWAP in terms of wind-wave age $C_{p} / U_{10}$.
\end{abstract}

Correspondence to: S. I. Badulin

(bsi@wave.sio.rssi.ru)

\section{Introduction}

To develop a proper and reliable theory that describes at least in some basic features the wind-driven sea is an urgent and challenging task. Very important step in this direction was done by Hasselmann $(1962,1963)$. Hasselmann started with the seminal work of Phillips (1960) who established that the main weakly nonlinear process in the system of gravity waves on the sea surface is a four-wave interaction governed by the following resonant conditions

$$
\left\{\begin{array}{l}
\boldsymbol{k}_{1}+\boldsymbol{k}_{2}=\boldsymbol{k}_{3}+\boldsymbol{k}_{4} \\
\omega_{1}+\omega_{2}=\omega_{3}+\omega_{4}
\end{array}\right.
$$

Starting from this point, Hasselmann $(1962,1963)$ derived the kinetic equation for squared amplitudes of water waves. This was an important achievement in fledging nonlinear physics as an outstanding event for physical oceanography. First, Hasselmann claimed that the theory of wind-driven sea can be treated as a part of theoretical physics: thus, the solution can be found by means of well-justified analytical and numerical methods. Then he persuaded the community of physical oceanographers to use his approach as a core of wave prediction models. Note, that the Hasselmann equation is just a limiting case of the quantum kinetic equation for phonons known in condensed matter physics since 1928 (Nordheim, 1928).

In spite of progress in development of new models for wave forecasting, this problem is far from being solved. Numerical solution of the Hasselmann equation turned out to be an extremely hard task for available computers. First attempts to solve this equation numerically were done in the seventies (Hasselmann and Hasselmann, 1981): since that time many researches all around the world contributed into development of algorithms and numerical codes (Hasselmann and Hasselmann, 1981, 1985; Webb, 1978; Masuda, 1980; Komatsu and Masuda, 1996; Polnikov, 1990, 1993; Lavrenov, 2003; Tracy and Resio, 1982; Resio and Perrie, 1991; Hashimoto et al., 2003). This list is not complete, it just shows the diversity of approaches to the problem. 
In parallel with numerical approaches, a number of models was proposed in order to substitute the exact Hasselmann equation by simpler (first of all, for numerical solution) models. The Discrete Interaction Approximation (DIA) is the first and the most known substitute of this kind (Hasselmann and Hasselmann, 1985). The main point of this approach is replacing the nonlinear integral operator $S_{n l}$ in the kinetic equation (collision integral) that describes a continuum of four-wave resonant interactions by a sum of relatively small number of terms for a particular set of resonant quadruplets (1). Recently the Multiple Discrete Interaction Approximation (MDIA) entered into practice as an extension of DIA, it is based on the same idea and on the power of modern computer systems (Hashimoto et al., 2003). Till now a "modus vivendi" in popular wave prediction models WAM (Komen et al., 1995) and SWAN (Booij et al., 1999) is the use of DIA and MDIA as substitutes for the kinetic equation.

An alternative approach is based on replacing the nonlinear integral operator $S_{n l}$ by a simpler differential operator. Hasselmann (Hasselmann et al., 1985) was the first who realized the idea of "differential approximation". Independently, Iroshnikov (1986) obtained the differential operator as an approximation for the collision integral. In both cases the forth-order differential operators were proposed. Later Zakharov and Pushkarev (1999) showed that the complicated fourth-order PDE's offered by Hasselmann and Iroshnikov can be simplified to the second-order nonlinear diffusion equations without loss of accuracy: the diffusion equations describe evolution of average spectra characteristics (energy, mean frequency) surprisingly well. To reach better description for fine features of spectra, several modifications of the simple diffusion model were offered (Pushkarev et al., 2004). In spite of this success, the simple models are not able to reproduce very important details of experimental spectra of wind-driven waves: the strong angular anisotropy that decreases with the frequency (angular spreading), the pronounced peakedness of the spectral distribution etc. As it has been pointed out recently by Benoit (2005) "the accuracy of currently used approximations (e.g. DIA and diffusion operators) are quite low" to approximate adequately the collision integral $S_{n l}$. Thus, the return to the exact kinetic Hasselmann equation seems inevitable.

Coming back to the Hasselmann equation we should stress that its derivation is based on a number of rather restrictive hypotheses and approximations. First of all, the phase randomness is suggested, i.e. the coherent structures like solitons and wave breaking do not play any role in the Hasselmann equation. Evidently, this condition is not fulfilled in some important physical situations. For example, in windwave tanks the wave turbulence spectrum is one-dimensional (quasi-one-dimensional) and the Hasselmann kinetic equation in its classical formulation is not valid. In this case more sophisticated theory is required, for instance, the approach offered recently by Janssen (2003) and developed successfully by Onorato et al. $(2004,2005)$ can be used.

The validity of kinetic description of large wave ensembles at long time was verified recently by several different ap- proaches to a direct numerical simulation of dynamical equations for water waves (Annenkov and Shrira, 2004a; Onorato et al., 2002; Dyachenko et al., 2004). In these papers the tendency to formation of weak-turbulent Kolmogorov spectra was demonstrated for discrete ensembles of deep water waves: the ensembles contained relatively small number of harmonics. Tanaka (2002) demonstrated the spectral maximum downshift that is the key physical effect of the kinetic theory of surface waves. Encouraging results in this direction are obtained recently by (Zakharov et al., 2005). in numerical experiments with regular ensembles of harmonics $(512 \times 512$ grid in wavevector space) and by Annenkov and Shrira (2005) for chains of resonant harmonics. However, an extensive verification of the Hasselmann kinetic equation by comparison with numerical solutions for dynamical equations is not carried out yet and remains a vital problem for the water waves study.

Taking into account the difficulties mentioned above, some researchers claim that the Hasselmann kinetic equation is not applicable to wind-driven waves (Rasmussen and Stiassnie, 1999). If this is the case, the vast activity of last decades focused on wave prediction models of the third and fourth generations is futile. We think that this opinion is too radical. Of course, such extremely important physical processes as wave breaking and freak wave formation remain beyond the kinetic equation. However, we believe that their contribution into the key effects - evolution of the mean energy and frequency of the spectral peak - is negligible or can be taken into account by adding some small correction terms to the kinetic equation. In other words, the wind wave spectrum near the pronounced spectral maximum is wide enough (in frequency and direction) for the kinetic equation to be valid.

The Hasselmann kinetic equation for water waves is not an extraordinary object of modern physics. Similar equations are well known in many other areas: in plasma physics, in helium superfluidity, in Bosé condensation problem etc. All these problems are subjects of the theory of weak turbulence. The key result of this theory is the exact KolmogorovZakharov (KZ) solutions of the stationary kinetic equation. These solutions describe cascades of motion constants to infinitely small or to infinitely large wave scales. The simplest "direct cascade" solution found by Zakharov and Filonenko (1966) is quite similar to the classical Kolmogorov spectrum of incompressible fluid turbulence and describes transport of energy from large to small scales where energy dissipates. The opposite tendency, also observed in the water wave ensembles, is the "inverse cascade" - the transport of wave action from small to large scales. The corresponding Kolmogorov-type solution was found by Zakharov in the same year (Zakharov, 1966) but was studied in details for water waves fifteen years later (Zakharov and Zaslavskii, 1982). Katz and Kontorovich (1971) showed that solutions of this type appear in a number of problems of weak turbulence (e.g. plasma waves) and found weakly anisotropic KolmogorovZakharov solutions (Katz and Kontorovich, 1974; Katz et al., 1975). 
Both these basic solutions imply sinks and sources at infinitely large or at infinitely small scales and no generation and dissipation in the domain of cascades, in the socalled inertial interval. In this case the nonlinear transfer is the only mechanism of wave field evolution. Existence of two Kolmogorov-type solutions that carry different constants of motion in two opposite directions takes place in two-dimensional turbulence of incompressible fluid (Kraichnan, 1967).

The development of weak turbulent theory proceeded in parallel with the boom in wind-wave forecasting based on the Hasselmann equation. Unfortunately, these two streams of science interacted quite weakly. In 1983 Kitaigorodskii, following the series of works by Zakharov and co-authors (Zakharov and Filonenko, 1966; Zakharov and Zaslavskii, 1982, 1983), tried to persuade the oceanographic community that the Kolmogorov-type approach is applicable to the winddriven sea. He was criticized by Komen et al. (1984), then by Phillips (1985), and the concept of Kolmogorov-type scenario was not accepted by majority of oceanographers. Criticism was based on superficial arguments: the main point was the difference in energy budgets for fully-developed turbulence in incompressible fluid and for the wind-driven weak turbulence of surface waves. Indeed, for sea waves wind input may not be concentrated in small wave numbers (as it is in 3-D turbulence of incompressible fluid) but is broadly distributed in the whole spectral range. However, this circumstance does not affect significantly the weak turbulence theory results and leads only to slow dependence of energy and wave action fluxes on wave scales. In spite of this dependence the weak turbulent theory correctly predicts exponents for wind-driven spectra tails as well as the rate of spectral peak downshift and the rate of energy growth with duration and fetch.

In 1985 Phillips formulated an alternative scenario for the energy budget. He argued that in the equilibrium range three major input terms in the Hasselmann equation - nonlinear transfer, wind input, and white-cap dissipation - are of the same order of magnitude and, thus, balance each other. Definitely, our numerical experiments do not support this viewpoint. They show that the nonlinear transfer term always surpasses income terms for growing wind-waves. This fact validates our approach. We have to stress once more that the validity of the kinetic equation for wind-driven waves is a problem of great concern and requires an extensive study of theoretical, experimental and numerical aspects in their intimate linkage. Phase-resolving models of wave field proposed recently (Annenkov and Shrira, 2005; Dyachenko et al., 2004; Onorato et al., 2002) have given indispensable tools of exploring this problem.

In this paper we apply systematically the weak-turbulent theory to the wind-driven sea. The wind-driven waves are generated by Cerenkov-type instability but it is not easy to calculate the growth rate of this instability. The atmospheric boundary layer near the sea surface is typically very turbulent, therefore, all theoretical models for instability, that is excited by a real air flow, are vulnerable for critics and can- not be justified without serious problems. Numerous experimental studies were carried out to determine the wind-wave generation rates. Actually, these complicated and very expensive experiments do not provide reliable estimates for the rates dealing with the effect of the wind. The dispersion of magnitudes for the wind input rates given by different authors is more than the rates themselves.

The situation with experimental background for the wave dissipation rates is even worse. Physical mechanisms of dissipation are still not clear, especially for long waves. At low winds, the generation of capillary waves is likely to be the major mechanism of dissipation. For moderate winds capillary waves generate micro-breakers that increase dramatically the dissipation in small wavelengths. The dissipation of dominant waves becomes noticeable for high winds (more than $6 \mathrm{~m} \cdot \mathrm{s}^{-1}$ ) when the breaking of waves occurs. Quantitative comparison of different dissipation mechanisms is not done yet, hence, all available formulas for nonlinear dissipation rates can be subject of criticism.

An additional unclear point is the following: how to separate wave generation and wave dissipation in the real sea experiments? In experiments the wave input is measured for special conditions of initial growth of short waves or by measuring the wave-induced pressure field above waves of any length (Plant, 1982). In the first case the nonlinear transfer and wave dissipation are assumed to be small. In the latter, the momentum and energy fluxes due to turbulent wind are thought to be absorbed completely by wind waves, drift currents, turbulence etc. The quantifying the fluxes to the various physical processes, certainly, requires sophisticated experimental setup or/and (rather and than or) additional hypotheses. Evidently, in this case the separation of wave growth and the dissipation is extremely difficult problem. In fact, less than $5 \%$ of the fluxes contribute into wave growth and the dissipation is generally measured as a residual quantity to provide a stationarity of wind-wave spectra in a particular spectral range (Komen et al., 1984). The effect of nonlinear transfer is ignored completely in this case (e.g. Eq.15 in Plant, 1982; Donelan and Pierson-jr., 1987; Tolman and Chalikov, 1996).

The key point of the theory we develop in this paper: Wind-wave growth does not depend on details of wind-wave generation and dissipation. We believe that this statement will be the starting point for further progress in our understanding the wind-driven waves. This statement requires a few notes. As far as the weak turbulent approach is relevant, the evolution of the wave spectra is governed by the following kinetic equation

$$
\frac{\partial N_{\boldsymbol{k}}}{\partial t}+\nabla_{\boldsymbol{k}} \omega_{\boldsymbol{k}} \nabla_{\boldsymbol{r}} N_{\boldsymbol{k}}=S_{n l}+S_{f}
$$

Here $S_{n l}$ is the nonlinear interaction term due to four-wave processes and

$S_{f}=S_{i n}+S_{d i s s}$.

describes non-conservative effects due to generation by wind-wave interaction $S_{i n}$ and dissipation $S_{d i s s}$. The indices 
of gradient symbols define the corresponding Fourier $(\boldsymbol{k})$ or coordinate $(\boldsymbol{r})$ spaces.

The main point is: in a short time (or in a short fetch) the collision integral $S_{n l}$ becomes greater than the nonconservative term $S_{f}$. Thus, in the first approximation Eq. (2) can be reduced up to the form

$$
\frac{\partial N_{k}}{\partial t}+\nabla_{k} \omega_{k} \nabla_{r} N_{k}=S_{n l}
$$

This equation looks similar to the Boltzmann equation for gas dynamics. However, there is a dramatic difference between these equations. The Boltzmann equation is complete and self-sustained. It has a unique solution at any initial data. Temporal evolution of this solution preserves energy, momentum, and total action (number of particles).

On the contrary, the Hasselmann kinetic Eq. (4) preserves energy and momentum "formally" only (Pushkarev et al., 2003, 2004). Due to presence of the Kolmogorov-type cascades, the energy and the momentum can "leak" at high wavenumber region. This region can also work as a source of wave action, energy and momentum. Thus, the conservative Eq. (4) is not "complete". It describes an "open system" and has to be accomplished by a "boundary condition", suppose, in the form of wave action flux at high frequencies. The full Eq. (2) with sufficiently large dissipation term at high frequencies appears to be well-posed. The former constants of motions cease to be constants but the balance equations for total wave action can be written trivially in the form

$$
\left\langle\frac{\partial N_{k}}{\partial t}+\nabla_{\boldsymbol{k}} \omega_{\boldsymbol{k}} \nabla_{\boldsymbol{r}} N_{\boldsymbol{k}}\right\rangle=\left\langle S_{f}\right\rangle
$$

where brackets mean the total wave action or the total wave forcing (integrated in the whole Fourier space). Similar balance equations can be written for energy and momentum.

Our basic statement is: the conservative kinetic Eq. (4) with the corresponding boundary conditions (Eq. 5) at $|\boldsymbol{k}| \rightarrow \infty$ is a fairly good approximation to the exact kinetic Eq. (2). The "effective" boundary condition is defined by the integral source term $\left\langle S_{f}\right\rangle$ and is not defined by the details of this term.

The analysis for the conservative kinetic Eq. (4) is incomparably simpler than the analysis for the full Eq. (2). The approximate Eq. (4) has a rich family of self-similar solutions. In this paper we discuss self-similar solutions of the "duration-limited" equation

$$
\frac{\partial N_{k}}{\partial t}=S_{n l}
$$

and of "fetch-limited" equation

$$
\nabla_{\boldsymbol{k}} \omega_{\boldsymbol{k}} \nabla_{\boldsymbol{r}} N_{\boldsymbol{k}}=S_{n l}
$$

Analytical properties of both classes of solutions are quite similar. In both cases we have two-parameter families of selfsimilar solutions. Parameters of these solutions are defined by the flux of wave action at $|\boldsymbol{k}| \rightarrow \infty$.
Using different versions of the source term $S_{f}$, we extend our analytical results with numerical analysis of the full duration-limited equation

$$
\frac{\partial N_{k}}{\partial t}=S_{n l}+S_{f}
$$

then solve this equation with different initial conditions: small "white" noise, step-like function of frequency, and JONSWAP spectral form. Irrespective of initial conditions we observe that nonlinear transfer term $S_{n l}$ starts to dominate in a very short time. Numerical solutions of the full Eq. (8) tend to self-similar solutions of the conservative Eq. (6) very rapidly. The corresponding indexes of self-similar solutions that are determined by boundary conditions (Eq. 5) (wave action flux from high wavenumbers) match the theoretical dependencies fairly well.

We compare the numerical results with experimental parameterizations of fetch-limited wind wave spectra, first of all, with the JONSWAP spectrum. Regardless quite different forms of the governing Eq. (7) the spectra appear to be surprisingly close to the self-similar solutions for durationlimited growth (Eq. 6). In addition, we find that shapes of numerical solutions depend very slightly on the indexes of self-similarity.

It is useful to reproduce main points of the paper in Introduction.

In Sect. 2 we present the "first principles" of weakly nonlinear approach for surface gravity waves. We follow strictly the Hamiltonian approach proposed by Zakharov (1968) (see also Zakharov, 1999; Krasitskii, 1994) for dynamical and statistical description of water waves.

In Sect. 3 we discuss experimental parameterizations of wind-driven waves spectra (first of all, the JONSWAP spectrum). These parameterizations postulate a universal dependence of spectra on non-dimensional frequency $\omega / \omega_{p}$ and monotonic dependence of the spectra magnitudes on the only parameter - the wave age $C_{p} / U_{\text {wind }}$. This is an implicit confirmation of our concept of spectral self-similarity. In the same section we discuss different models for the input $\left(S_{i n}\right)$ and dissipation $\left(S_{\text {diss }}\right)$ terms and compare these terms with the collision integral $S_{n l}$. For this comparison we use experimentally observed spectra obtained in JONSWAP experiments. Simple calculations show that for all conventional models of $S_{f}=S_{i n}+S_{d i s s}$ the nonlinear term $S_{n l}$ is strongly dominating.

Section 4 is devoted to weak-turbulent Kolmogorov spectra (or the Kolmogorov-Zakharov spectra). We show that in general case the spectra are governed by three independent parameters: the fluxes of wave action, energy, and momentum, i.e. are defined by a function of three variables. In the simplest case this function can be found in the explicit form. Quantitative dependence of spectra on fluxes is defined by fundamental constants, the so-called Kolmogorov constants. We finalize the section by qualitative analysis of the effect of spectrally distributed forcing and dissipation on fluxes and, hence, on spectral forms of stationary solutions of the kinetic equation. 
In Sect. 5 we introduce self-similar solutions for durationlimited and fetch-limited versions of the Hasselmann kinetic equation. In proper variables, self-similar spectra satisfy the stationary homogenous kinetic equation with some sophisticated $S_{f}$ that, formally, includes forcing and dissipation and, thus, can arrest the downshift. The structure of the selfsimilar solutions is rather complicated. They are anisotropic and are determined, at least, by two free parameters. All these solutions (excluding special case of swell) correspond to wave action flux from high frequency region. Being integrated in $\boldsymbol{k}$-space this flux is a power-like function of time (fetch) which exponent and pre-exponent can be related easily with parameters of the self-similar solutions. It is easy to show that the solutions for special cases of the self-similarity indexes tend to the Kolmogorov-Zakharov stationary spectra at $t \rightarrow \infty$.

In Sect. 6 the results of numerical solutions of the kinetic equation are presented. We refer to our previous papers (Pushkarev et al., 2003; Badulin et al., 2002) in a brief description of numerical approach. Then we analyze the results of the so-called "academic" numerical experiments on duration-limited growth of wind-driven waves. The purpose of the "academic" experiments is to justify theoretical results of previous section and to demonstrate the tendency of the kinetic equation solutions to self-similar behavior. In addition, these somewhat artificial experiments serve as a reference for experiments with realistic conditions of wind-wave evolution.

A number of numerical experiments was performed for the duration-limited Hasselmann Eq. (8) with "realistic" wind input parameterizations (Snyder et al., 1981; Stewart, 1974; Plant, 1982; Hsiao and Shemdin, 1983; Donelan and Piersonjr., 1987). Our analysis is based essentially on theoretical analysis of self-similarity (Sect. 5) and "academic" runs described in Sect. 6. In terms of non-dimensional parameters non-dimensional frequency $\omega / \omega_{p}\left(\omega_{p}\right.$ is a frequency of spectrum peak) and wave age $g /\left(U_{10} \omega_{p}\right)$ - we found very good agreement for all considered parameterizations of wind wave input. Different criteria of the agreement could be applied for comparison. First, we analyze the agreement in terms of selfsimilarity indexes, then in terms of the shapes of solutions. All solutions appear to be very close to the shapes of "academic" solutions and are reasonably close to experimental parameterizations of JONSWAP.

Properties of self-similarity of numerical solutions in terms of fluxes of motion constants are presented in Sect. 7. This analysis shows that the self-similar solutions can be considered as a generalization of the Kolmogorov-Zakharov solutions: the generic feature of the KZ cascading - a rigid dependence of spectral magnitudes on spectral fluxes - keeps validity for the non-stationary (inhomogeneous) anisotropic solutions.

In Sect. 8 we present an overview of our results and their possible applications for the problem of wind-wave forecasting.

\section{Weakly nonlinear approach for water waves - Back- ground and definitions}

\subsection{Dynamical equations for water waves}

Equations for potential wave motion in incompressible infinitely deep water with a free surface can be written in terms of velocity potential $\Phi(\boldsymbol{x}, z, t)$ and surface elevation $\eta(\boldsymbol{x}, t)$ as follows

$$
\begin{aligned}
& \triangle \Phi=0 ; \quad-\infty<z<\eta(\boldsymbol{x}, t) \\
& \frac{\partial \eta}{\partial t}+\nabla \Phi \nabla \eta=-\frac{\partial \Phi}{\partial z} \quad z=\eta(\boldsymbol{x}, t) \\
& \frac{\partial \Phi}{\partial t}+g \eta+\frac{1}{2}(\nabla \Phi)^{2}+\frac{1}{2} \Phi_{z}^{2}+P=0 \quad z=\eta(\boldsymbol{x}, t)
\end{aligned}
$$

We split coordinates to the horizontal wavevector $\boldsymbol{x}$ and the vertical coordinate $z$. Here the Laplace equation (Eq. 9) comes from the condition of incompressibility, the kinematical boundary condition (Eq. 10) describes continuity of the surface elevation, and the dynamical boundary condition (Eq. 11) corresponds to continuity of pressure across water surface. As shown by Zakharov (1968), Eqs. (9)-(11) can be presented in the Hamiltonian form for two canonically conjugated variables $\eta$ and $\psi$

$\frac{\partial \eta}{\partial t}=\frac{\delta H}{\delta \psi} ; \quad \frac{\partial \psi}{\partial t}=-\frac{\delta H}{\delta \eta}$

Here the canonical coordinate $\eta(\boldsymbol{x}, t)$ is the surface elevation and the canonical conjugated coordinate defined as

$\psi(\boldsymbol{x}, t)=\Phi(\boldsymbol{x}, \eta(\boldsymbol{x}, t), t)$

is the velocity potential at the water surface. Symbol $\delta$ in Eq. (12) is used for variational (Fréchet) derivative. Further transformation to the Fourier amplitudes of $\eta$ and $\psi$ conserves the Hamiltonian form of Eq. (12) because of canonicity of the Fourier transform. Define this transformation as follows

$$
\begin{array}{ll}
\eta(\boldsymbol{x})=\frac{1}{2 \pi} \int \eta(\boldsymbol{k}) \exp (\mathrm{i} \boldsymbol{k} \boldsymbol{x}) d \boldsymbol{k}, & \eta(\boldsymbol{k})=\eta^{*}(-\boldsymbol{k}) ; \\
\psi(\boldsymbol{x})=\frac{1}{2 \pi} \int \psi(\boldsymbol{k}) \exp (\mathrm{i} \boldsymbol{k} \boldsymbol{x}) d \boldsymbol{k}, & \psi(\boldsymbol{k})=\psi^{*}(-\boldsymbol{k})
\end{array}
$$

While the Hamiltonian and the canonical variables $\psi, \eta$ are real-valued, one can introduce complex normal variables. The conventional definition of these variables (Zakharov, 1968; Krasitskii, 1994)

$$
\begin{aligned}
& \eta(\boldsymbol{k})=\Upsilon(\boldsymbol{k})\left[a(\boldsymbol{k})+a^{*}(-\boldsymbol{k})\right] \\
& \psi(\boldsymbol{k})=-\mathrm{i} \Lambda(\boldsymbol{k})\left[a(\boldsymbol{k})-a^{*}(-\boldsymbol{k})\right] \\
& \Upsilon(\boldsymbol{k})=\left[\frac{|\boldsymbol{k}|}{2 \omega(\boldsymbol{k})}\right]^{1 / 2} ; \quad \Lambda(\boldsymbol{k})=\left[\frac{\omega(\boldsymbol{k})}{2|\boldsymbol{k}|}\right]^{1 / 2} \\
& \omega(\boldsymbol{k})=[g|\boldsymbol{k}| \tanh (|\boldsymbol{k}| H)]^{1 / 2}
\end{aligned}
$$


is used in numerous papers on the Hamiltonian weakly nonlinear approach. Introduce the alternative definition of the normal variables

$$
\begin{aligned}
& \eta(\boldsymbol{k})=2 \pi \Upsilon(\boldsymbol{k})\left[A(\boldsymbol{k})+A^{*}(-\boldsymbol{k})\right] ; \\
& \psi(\boldsymbol{k})=-2 \pi \mathrm{i} \Lambda(\boldsymbol{k})\left[A(\boldsymbol{k})-A^{*}(-\boldsymbol{k})\right]
\end{aligned}
$$

or, simpler as

$$
A(\boldsymbol{k})=\frac{1}{2 \pi} a(\boldsymbol{k})
$$

This emphasis on notation may seem somewhat trivial but different notations can lead to mutual misunderstanding and grave mistakes. The advantage of normalization (Eq. 14) comes from the linear wave theory that gives

$$
\begin{aligned}
& \eta(\boldsymbol{x}, t)=\sqrt{\frac{2|\boldsymbol{k}|}{\omega(\boldsymbol{k})}} A_{0} \cos \left(\boldsymbol{k} \boldsymbol{x}-\omega(\boldsymbol{k}) t-\theta_{0}\right), \\
& \psi(\boldsymbol{x}, t)=\sqrt{\frac{2 \omega(\boldsymbol{k})}{|\boldsymbol{k}|}} A_{0} \sin \left(\boldsymbol{k} \boldsymbol{x}-\omega(\boldsymbol{k}) t-\theta_{0}\right)
\end{aligned}
$$

for the simplest plane wave solution and the simple expression for the plane wave energy

$$
E=\omega A_{0}^{2}
$$

By definition, $A_{0}^{2}=E / \omega$ is the density of wave action. The definition of normal variables $a(\boldsymbol{k})$ (Eq. 13) is common for theoretical studies while $A(\boldsymbol{k})$ (Eq. 14) are useful for practical needs such as wave forecasting.

Starting with linear approximation one can obtain the following explicit expression for the Hamilton function $H$ in terms of integral power series in normal amplitudes $a(\boldsymbol{k})$

$H=H_{0}+H_{1}+H_{2} \ldots$

where

$$
\begin{aligned}
H_{0} & =\frac{1}{2} \int V_{012}^{(1)}\left(a_{0}^{*} a_{1} a_{2}+\text { c.c. }\right) \delta_{0-1-2} d \boldsymbol{k}_{012} \\
H_{1} & =\frac{1}{3} \int V_{012}^{(3)}\left(a_{0} a_{1} a_{2}+\text { c.c. }\right) \delta_{0+1+2} d \boldsymbol{k}_{012} \\
& +\frac{1}{2} \int T_{0123}^{(1)}\left(a_{0}^{*} a_{1} a_{2} a_{3}+\text { c.c. }\right) \delta_{0-1-2-3} d \boldsymbol{k}_{0123} \\
H_{2} & =\frac{1}{4} \int T_{0123}^{(2)}\left(a_{0}^{*} a_{1}^{*} a_{2} a_{3}+\text { c.c. }\right) \delta_{0+1-2-3} d \boldsymbol{k}_{0123} \\
& +\frac{1}{8} \int T_{0123}^{(4)}\left(a_{0}^{*} a_{1}^{*} a_{2}^{*} a_{3}^{*}+\text { c.c. }\right) \delta_{0+1+2+3} d \boldsymbol{k}_{0123}
\end{aligned}
$$

We follow the kernel notations by Zakharov (1999) and the abbreviations for arguments by Krasitskii (1994). Notation "c.c." means complex conjugate terms. Corresponding dynamical equations for variables $a(\boldsymbol{k})$

$\mathrm{i} \frac{\partial a(\boldsymbol{k})}{\partial t}=\frac{\delta H}{\delta a^{*}(\boldsymbol{k})}$

contain a number of "unessential" terms. Both cubic terms with kernel $V^{(3)}$ and quartic terms with $T^{(1)}$ and $T^{(3)}$ are not resonant: they correspond to slave harmonics. Slave harmonics are corrections to linear approximation solutions that we call master modes. Slave harmonics are strongly linked with master modes. One can perform a canonical transformation to new variables $b(\boldsymbol{k})$ in order to cumulate slave harmonics in dynamically essential master modes. The general form of the transformation (Krasitskii, 1994; Zakharov, 1999)

$$
\begin{aligned}
a_{0} & =b_{0}+\int A_{012}^{(1)} b_{1} b_{2} \delta_{0-1-2} d \boldsymbol{k}_{12} \\
& +\int A_{012}^{(2)} b_{1}^{*} b_{2} \delta_{0+1-2} d \boldsymbol{k}_{12} \\
& +\int A_{012}^{(3)} b_{1}^{*} b_{2}^{*} \delta_{0+1+2} d \boldsymbol{k}_{12} \\
& +\int B_{0123}^{(1)} b_{1} b_{2} b_{3} \delta_{0-1-2-3} d \boldsymbol{k}_{123} \\
& +\int B_{0123}^{(2)} b_{1}^{*} b_{2} b_{3} \delta_{0+1-2-3} d \boldsymbol{k}_{123} \\
& +\int B_{0123}^{(3)} b_{1}^{*} b_{2}^{*} b_{3} \delta_{0+1+2-3} d \boldsymbol{k}_{123} \\
& +\int B_{0123}^{(4)} b_{1}^{*} b_{2}^{*} b_{3}^{*} \delta_{0+1+2+3} d \boldsymbol{k}_{123}+O\left(b^{4}\right)
\end{aligned}
$$

is cumbersome but makes possible to simplify essentially the dynamic Eq. (17) and the Hamiltonian function (Eq. 16). This transformation cancels a number of non-resonant terms in Eq. (16) and leads to the effective Hamiltonian

$$
\begin{aligned}
H\left(b_{\boldsymbol{k}}, b_{\boldsymbol{k}}^{*}\right) & =\int \omega_{\boldsymbol{k}} b_{\boldsymbol{k}} b_{\boldsymbol{k}}^{*} d \boldsymbol{k} \\
& +\frac{1}{4} \int \tilde{T}_{0123}^{(2)} b^{*} b_{1}^{*} b_{2} b_{3} \delta_{0+1-2-3} d \boldsymbol{k}_{0123}
\end{aligned}
$$

and to the equation known as the Zakharov equation (Zakharov, 1968)

$$
\begin{aligned}
\mathrm{i} \frac{\partial b_{\boldsymbol{k}}}{\partial t}=\frac{\partial H}{\partial b_{\boldsymbol{k}}^{*}} & =\omega_{\boldsymbol{k}} b_{\boldsymbol{k}} \\
& +\frac{1}{2} \int \tilde{T}_{0123}^{(2)} b_{1}^{*} b_{2} b_{3} \delta_{0+1-2-3} d \boldsymbol{k}_{123}
\end{aligned}
$$

Explicit expressions for the coefficients of the Hamilton function and for canonical transformation (Eq. 18) can be found in (Krasitskii, 1994; Zakharov, 1999) and in Appendix A.

In terms of master modes, the classic Stokes solution for deep water waves looks trivial

$$
\begin{aligned}
b_{\text {Stokes }} & =b_{0} \exp (i \Omega t) \delta\left(\boldsymbol{k}-\boldsymbol{k}_{0}\right) \\
\Omega & =\omega(\boldsymbol{k})+\frac{1}{2} T_{0000}\left|b_{0}\right|^{2}
\end{aligned}
$$

Here $b_{0}=$ const and the superscript for kernel $T^{(2)}$ is omitted. This gives the well-known Stokes expansion for the surface elevation

$$
\begin{aligned}
\eta(\boldsymbol{x}) & =-\frac{1}{2 \pi} \sqrt{\frac{2\left|\boldsymbol{k}_{0}\right|}{\omega\left(\boldsymbol{k}_{0}\right)}} b_{0} \cos \left(\Omega t-\boldsymbol{k}_{0} \boldsymbol{x}-\theta_{0}\right) \\
& +\frac{1}{2 \pi^{2}}\left(\frac{\left|\boldsymbol{k}_{0}\right|}{\omega\left(\boldsymbol{k}_{0}\right)}\right)^{2} b_{0}^{2} \cos \left[2\left(\Omega t-\boldsymbol{k}_{0} \boldsymbol{x}-\theta_{0}\right)\right]+O\left(b_{0}^{3}\right)
\end{aligned}
$$


that can be rewritten easily in conventional terms of the amplitude of the first linear harmonic (compare Eq. 15)

$$
\begin{aligned}
\eta(\boldsymbol{x}) & =-\eta_{0} \cos \left(\Omega t-\boldsymbol{k}_{0} \boldsymbol{x}-\theta_{0}\right) \\
& +\frac{\left|\boldsymbol{k}_{0}\right| \eta_{0}^{2}}{2} \cos \left[2\left(\Omega t-\boldsymbol{k}_{0} \boldsymbol{x}-\theta_{0}\right)\right]+O\left(\eta_{0}^{3}\right)
\end{aligned}
$$

where

$\Omega=\omega\left[1+\left(\eta_{0}|\boldsymbol{k}|\right)^{2} / 2\right]$

As we see, the weak nonlinearity leads to weak correction of linear frequency $\omega(\boldsymbol{k})$ in the Stokes solution (Eqs. 21 and 22) and to appearance of new slave harmonics. Evidently, mean values such as mean energy, wave action etc. are corrected in a similar way.

\subsection{Statistical description - the Hasselmann equation}

The Hasselmann kinetic equation is the basic theoretical model for statistical description of gravity surface waves. This equation can be obtained within the Hamiltonian approach in a consistent way. A few important notes should be made for the correct treatment of this equation.

The basic assumption of weak nonlinearity (small wave steepness)

$\mu=a k \ll 1$

is usually considered as a validity condition of the Hasselmann equation for water waves. Sea waves are weakly nonlinear, their typical steepness is less than 0.1 even in severe storm conditions and much less than critical water wave steepness

$\mu_{c r}=0.4019$

Assuming the wave field as a superposition of statistically independent harmonics (that is true in linear wave approximation) one can define the spectral wave action density in terms of alternative normal variables $A(\boldsymbol{k})$ (Eq. 14)

$N(\boldsymbol{k})=\frac{1}{g}\left\langle A(\boldsymbol{k}) A^{*}\left(\boldsymbol{k}^{\prime}\right)\right\rangle \delta\left(\boldsymbol{k}-\boldsymbol{k}^{\prime}\right)$

According to "oceanographic definition", the spectral wave energy density is

$E(\boldsymbol{k})=\frac{\omega(\boldsymbol{k})}{g}\left\langle A(\boldsymbol{k}) A^{*}\left(\boldsymbol{k}^{\prime}\right)\right\rangle \delta\left(\boldsymbol{k}-\boldsymbol{k}^{\prime}\right)$

Spectral densities (Eqs. 23 and 24) are known as asymmetric spectra. Their symmetric counterparts can be introduced as

$I(\boldsymbol{k})=\frac{1}{2} \omega(\boldsymbol{k})[N(\boldsymbol{k})+N(-\boldsymbol{k})]$

and

$\left\langle\eta(\boldsymbol{k}) \eta\left(\boldsymbol{k}^{\prime}\right)\right\rangle=I(\boldsymbol{k}) \delta\left(\boldsymbol{k}+\boldsymbol{k}^{\prime}\right)$

Then the wave amplitude dispersion $\sigma$ is given by evident formula

$\sigma^{2}=\left\langle\eta^{2}\right\rangle=\int \omega(\boldsymbol{k}) N(\boldsymbol{k}) d \boldsymbol{k}=\int I(\boldsymbol{k}) d \boldsymbol{k}$
The weak nonlinearity assumption must be completed by hypothesis on wave field uniformity. Thus, the correlation function for normal variables contains $\delta$-functions (see Eqs. 23 and 25). This is true for presentation both in variables $a(\boldsymbol{k})$ and in master modes $b(\boldsymbol{k})$ :

$$
\begin{aligned}
\left\langle a(\boldsymbol{k}) a^{*}\left(\boldsymbol{k}^{\prime}\right)\right\rangle & =n_{a}(\boldsymbol{k}) \delta\left(\boldsymbol{k}-\boldsymbol{k}^{\prime}\right) \\
\left\langle b(\boldsymbol{k}) b^{*}\left(\boldsymbol{k}^{\prime}\right)\right\rangle & =n_{b}(\boldsymbol{k}) \delta\left(\boldsymbol{k}-\boldsymbol{k}^{\prime}\right)
\end{aligned}
$$

The same is true for "observable" $A(\boldsymbol{k})$ and its "master" counterpart. Similarly to the dynamical description, the difference between $n_{a}$ and $n_{b}$ is small

$\frac{\left|n_{a}-n_{b}\right|}{n_{a}} \simeq \mu^{2}$

and can be ignored in a number of cases. However, this is correct for the infinite depth case only (Zakharov, 1999). It should be stressed that the kinetic equation is derived for correlation function $n_{b}(\boldsymbol{k})$, that is, for the master mode decomposition of wave field as it was introduced above. Hereafter we omit the subscript for $n(\boldsymbol{k})$ and $N(\boldsymbol{k})$.

The kinetic equation requires the closure hypothesis imposed on correlation functions. We assume that

$<b_{1} \ldots b_{n} b_{n+1}^{*} \ldots b_{n+m}^{*}>=0$ if $m \neq n$

It is easy to check that this assumption is compatible with Eq. (19). Correlation functions

$$
\begin{aligned}
& <b_{1} \ldots b_{n} b_{n+1}^{*} \ldots b_{2 n}^{*}> \\
& =I_{1, \ldots, n, n+1, \ldots, 2 n} \cdot \delta\left(k_{1}+\cdots+k_{n}-k_{n+1}-\cdots-k_{2 n}\right)
\end{aligned}
$$

can be decomposed by a standard way to a sum of products of some low-order functions and cumulants. In particular,

$$
I_{1234}=n_{1} n_{2}\left[\delta\left(\boldsymbol{k}_{1}-\boldsymbol{k}_{3}\right)+\delta\left(\boldsymbol{k}_{1}-\boldsymbol{k}_{4}\right)\right]+\tilde{I}_{1234}
$$

where $\tilde{I}_{1234}$ is irreducible part of the forth-order cumulant. The closure hypothesis claims that the irreducible parts of the next six-order cumulants are plain zeroes and, thus, the imaginary part of $\tilde{I}_{1234}$ can be expressed following Zakharov (1999) as follows

$\operatorname{Im}\left(\tilde{I}_{1234}\right)=\pi T_{1234}\left[n_{3} n_{4}\left(n_{2}+n_{1}\right)-n_{1} n_{2}\left(n_{3}+n_{4}\right)\right]$

This hypothesis leads to the Hasselmann kinetic equation that we will write for $N(\boldsymbol{k})=n(\boldsymbol{k}) /\left(4 \pi^{2}\right)$

$\frac{\partial N_{\boldsymbol{k}}}{\partial t}+\nabla_{\boldsymbol{k}} \omega_{\boldsymbol{k}} \nabla_{\boldsymbol{r}} N_{\boldsymbol{k}}=S_{n l}+S_{i n}+S_{\text {diss }}$

Here the collision integral has the form

$$
\begin{aligned}
S_{n l} & =16 \pi^{5} g^{2} \int\left|T_{0123}\right|^{2} \\
& \times\left(N_{1} N_{2} N_{3}+N_{0} N_{2} N_{3}-N_{0} N_{1} N_{2}-N_{0} N_{1} N_{3}\right) \\
& \times \delta\left(\omega_{0}+\omega_{1}-\omega_{2}-\omega_{3}\right) \\
& \times \delta\left(\boldsymbol{k}+\boldsymbol{k}_{1}-\boldsymbol{k}_{2}-\boldsymbol{k}_{3}\right) d \boldsymbol{k}_{1} d \boldsymbol{k}_{2} d \boldsymbol{k}_{3}
\end{aligned}
$$

Note, that inhomogeneity of $N(\boldsymbol{k})$ is assumed to be weak in Eq. (27), i.e. the wave field uniformity conditions (Eq. 26) are satisfied "locally" and the collision integral does not depend explicitly on coordinates. Cumbersome expressions for 
the kernel $T_{0123}$ can be found in a number of papers (see Hasselmann, 1962; Zakharov, 1968; Webb, 1978; Krasitskii, 1994). Different forms of $T_{0123}$ can be used as far as the kernel is unique in the resonant subspace and is arbitrary beyond the subspace. The collection of formulas is given in Appendix A.

The most important fact we will use further: in the deep water case the kernel $T\left(\boldsymbol{k}_{0}, \boldsymbol{k}_{1}, \boldsymbol{k}_{2}, \boldsymbol{k}_{3}\right)$ is a homogeneous function of order six:

$\left|T\left(\kappa \boldsymbol{k}, \kappa \boldsymbol{k}_{1}, \kappa \boldsymbol{k}_{2}, \kappa \boldsymbol{k}_{3}\right)\right|^{2}=\kappa^{6}\left|T\left(\boldsymbol{k}, \boldsymbol{k}_{1}, \boldsymbol{k}_{2}, \boldsymbol{k}_{3}\right)\right|^{2}$

This gives the well-known re-scaling property of the collision integral for deep water waves

$S_{n l} \simeq g^{3 / 2}|\boldsymbol{k}|^{19 / 2} N_{*}^{3}$

where $N_{*}$ is a "characteristic" scale of wave action density, say, its peak or mean value.

2.3 Notes on canonical and oceanographer's definition of wind wave spectra

Let us introduce notations and definitions that we use further in this paper. We accept "oceanographer's" definition of the total wave energy as follows

$E=\int_{\boldsymbol{k}} E(\boldsymbol{k}) d \boldsymbol{k}=\int_{0}^{+\infty} \int_{-\pi}^{+\pi} E(\omega, \theta) d \omega d \theta=\left\langle\eta^{2}\right\rangle$

In these terms the total energy has dimension $\left[L^{2}\right]$, the energy spectral density $E(\boldsymbol{k})-\left[L^{4}\right]$ and the frequency spectral density $E(\omega, \theta)-\left[L^{2} T\right]$. We refer to the spectral density of wave action $N(\boldsymbol{k}, t)$ as a solution for the kinetic equation (27). Following Eq. (30) one gets

$N=\int \frac{E(\boldsymbol{k}, t)}{\omega(\boldsymbol{k})} d \boldsymbol{k}=\int N(\boldsymbol{k}, t) d \boldsymbol{k}$

where the wave action spectral density has dimension $\left[L^{4} T\right]$. The wave action densities in frequency-angle space are defined by evident relations obtained from equivalence of the corresponding differentials

$$
\begin{aligned}
N(\omega, \theta) d \omega d \theta & =N(\boldsymbol{k}, t)|\boldsymbol{k}| d|\boldsymbol{k}| d \theta \\
& =N(\boldsymbol{k}, t)|\boldsymbol{k}| \frac{\partial|\boldsymbol{k}|}{\partial \omega} d \omega d \theta
\end{aligned}
$$

Then for the deep water waves one has

$N(\omega, \theta)=\frac{2 \omega^{3}}{g^{2}} N(\boldsymbol{k})$

Energy spectral density can be introduced quite similarly

$E(\omega, \theta) d \omega d \theta=\omega(\boldsymbol{k}) N(\boldsymbol{k}) d \boldsymbol{k}=\frac{2 \omega^{4}}{g^{2}} N(\omega, \theta) d \omega d \theta$

Later we refer to frequency one-dimensional spectra as functions of one argument only

$N(\omega)=\int_{-\pi}^{\pi} N(\omega, \theta) d \theta ; \quad E(\omega)=\int_{-\pi}^{\pi} E(\omega, \theta) d \theta$

\subsection{On fine structure of $S_{n l}$}

Estimate Eq. (29) is very rough and applicable only in vicinity of the spectral peak for smooth spectral distributions similar to the Pierson-Moskowitz spectrum. Usual spectra have power-like "tails" for $|\boldsymbol{k}|>\left|\boldsymbol{k}_{p}\right|$. To estimate $S_{n l}$ on such rare face of the spectral peak one has to present the collision integral as

$S_{n l}=F_{k}-\gamma_{k} N_{k}$

where

$$
\begin{aligned}
F_{\boldsymbol{k}} & =16 \pi^{5} g^{2} \int\left|T_{0123}\right|^{2} \\
& \times N_{1} N_{2} N_{3} \delta\left(\boldsymbol{k}+\boldsymbol{k}_{1}-\boldsymbol{k}_{2}-\boldsymbol{k}_{3}\right) \\
& \times \delta\left(\omega_{\boldsymbol{k}}+\omega_{1}-\omega_{2}-\omega_{3}\right) d \boldsymbol{k}_{1} d \boldsymbol{k}_{2} d \boldsymbol{k}_{3} \\
\gamma_{\boldsymbol{k}} & =16 \pi^{5} g^{2} \int\left|T_{0123}\right|^{2}\left(N_{1} N_{2}+N_{1} N_{3}-N_{2} N_{3}\right) \\
& \times \delta\left(\boldsymbol{k}+\boldsymbol{k}_{1}-\boldsymbol{k}_{2}-\boldsymbol{k}_{3}\right) \\
& \times \delta\left(\omega_{\boldsymbol{k}}+\omega_{1}-\omega_{2}-\omega_{3}\right) d \boldsymbol{k}_{1} d \boldsymbol{k}_{2} d \boldsymbol{k}_{3}
\end{aligned}
$$

From a physical view-point $\gamma_{\boldsymbol{k}}$ is the imaginary part of frequency that appears due to four-wave nonlinear interaction or inverse relaxation time $\tau^{-1}$. On the rear faces of the spectral peak $N_{\boldsymbol{k}} \simeq|\boldsymbol{k}|^{-s}, s \simeq 4$. For these powerlike spectra, integrals (Eqs. 32 and 33) diverge at small wavenumbers and major contribution to these integrals is given by a vicinity of the spectral peak. For $|\boldsymbol{k}| \gg\left|\boldsymbol{k}_{p}\right|, \gamma_{\boldsymbol{k}}$ can be estimated as follows

$\gamma_{\boldsymbol{k}} \simeq 32 \pi^{5} g^{2} \int\left|T_{\boldsymbol{k} \boldsymbol{k}_{p} \boldsymbol{k} \boldsymbol{k}_{p}}\right|^{2} N_{1} N_{2} \delta\left(\omega_{1}-\omega_{2}\right) d \boldsymbol{k}_{1} d \boldsymbol{k}_{2}$

For $\left|\boldsymbol{k}_{p}\right|<|\boldsymbol{k}|$ one has (see Lavrova, 1983)

$T_{\boldsymbol{k}_{p}} \boldsymbol{k} \boldsymbol{k}_{p}=\frac{1}{4 \pi^{2}}\left|\boldsymbol{k}_{p}\right|^{2}|\boldsymbol{k}|$

that gives

$\gamma_{\boldsymbol{k}} \simeq \frac{2 \pi \omega^{4}}{\omega_{p}^{2} \delta \omega}\left(\left|\boldsymbol{k}_{p}\right|^{2} E\right)^{2}$

One can see that $\gamma_{k}$ is a fast growing function of $\omega$ and

$\gamma_{\boldsymbol{k}} N_{\boldsymbol{k}} \simeq|\boldsymbol{k}|^{-s+2}$

At the same time the "naive" estimate Eq. (29) gives

$S_{n l} \simeq \Lambda(s)|\boldsymbol{k}|^{\frac{19}{2}-3 s}$

where $\Lambda(s)$ is a function of the exponent $s$ of the spectrum tail. For a typical case $s=4$, one has

$\gamma_{\boldsymbol{k}} N_{\boldsymbol{k}} \simeq|\boldsymbol{k}|^{-2} ; \quad S_{n l} \simeq \Lambda(s)|\boldsymbol{k}|^{-5 / 2}$

Thus, $\gamma_{\boldsymbol{k}} N_{\boldsymbol{k}}$ decays at $|\boldsymbol{k}| \rightarrow \infty$ slower than $S_{n l}$.

Actually in the "inertial range" different $S_{n l}$ terms (see Eq. 31) compensate each other. As a result, divergences at small wavenumbers are exactly cancelled. The dimensionless factor $\Lambda(s)$ depends strongly on $s$ and in the case of 
isotropic spectra becomes plain zero for the Kolmogorov exponents $s=4, s=23 / 6$ :

$\Lambda(4)=\Lambda\left(\frac{23}{6}\right)=0$

We can make the following conclusion: estimate Eq. (29) can lead to a grave mistake for the inertial range. In this range $S_{n l} \rightarrow 0$, while different parts of $S_{n l}$ are definitely not zeroes.

In many cases the spectrum $N(\boldsymbol{k})$ is narrow near the spectral peak (has "peakedness"). Then, estimate Eq. (29) is also not valid and should be replaced by the following one

$S_{n l} \simeq \Lambda g^{3 / 2}|\boldsymbol{k}|^{19 / 2} N^{3}$

Here $\Lambda$ is a large dimensionless constant $(\Lambda \gg 1)$ and can be called "enhancing factor". To calculate the enhancing factor, we suppose the spectral width to be small $\left(\delta \omega \ll \omega_{p}\right)$. Near the peak one can perform the following replacement

$$
\begin{gathered}
\delta\left(\omega_{0}+\omega_{1}-\omega_{2}-\omega_{3}\right) \rightarrow \\
\frac{2}{\omega_{\boldsymbol{k} \boldsymbol{k}}^{\prime \prime}} \delta\left((d \boldsymbol{k})^{2}+\left(d \boldsymbol{k}_{1}\right)^{2}-\left(d \boldsymbol{k}_{2}\right)^{2}-\left(d \boldsymbol{k}_{3}\right)^{2}\right)
\end{gathered}
$$

Moreover, for the "peaked" spectrum the mean frequency $\bar{\omega}$ differs from the peak frequency $\omega_{p}$. Taking into account both factors, one obtains the following expression for $\Lambda$ from Eq. (29):

$$
\Lambda \sim \frac{\omega_{p}}{\delta \omega}\left(\frac{\bar{\omega}}{\omega_{p}}\right)^{9}
$$

The typical ratio $\bar{\omega} / \omega_{p} \simeq 1.25$ is close to unity but its ninth power is not a small factor: $\left(\bar{\omega} / \omega_{p}\right)^{9} \approx 7.45$. Our calculation shows that the enhancing factor $\Lambda$ can be of order $\Lambda \simeq 50 \div 100$ near the spectral peak, while in the inertial range $\Lambda \rightarrow 1$.

\section{On dominant role of nonlinear interactions in Hassel- mann equation}

In this section we give an overview of different parameterizations for wind-wave spectra and for generation terms in the Hasselmann equation. These parameterizations accumulate essential features of wind-wave behavior in the most compact form and can be adequately related to the "first principles" presented above.

First we consider JONSWAP parameterizations of windwave spectra that will be used further as a basis for comparison of experimental and numerical spectra. In addition, we pay attention to the self-similarity features of the JONSWAP parameterization that is the key point of the study. Actually, the idea of self-similarity of the wind-wave spectra was introduced by experimentalists (see Kitaigorodskii, 1962) long before its theoretical understanding (Hasselmann et al., 1973).

Conventional parameterizations of input and dissipation terms in the Hasselmann equation are based on rather poor quantitative knowledge of different physical processes that govern the wind-wave evolution (wind-wave interaction, turbulence and mixing in sea upper layer etc). Empirical dependencies proposed by different authors differ from each other: the difference is at least of the same order as the magnitudes themselves. Thus, the problem arises: how to choose a "true" forcing (input and generation) term?

In the final part of the section the collision integral $S_{n l}$ and source functions $S_{i n}$ and $S_{d i s s}$ are compared. This comparison does not require the solution of the evolution problem: it is enough to make "snapshots" of terms for different experimentally measured spectra. We used JONSWAP spectra for the comparison and came to the following basic statement regarding the wind-wave evolution: as compared to windwave generation and dissipation, the nonlinearity dominates in rather wide range of physical conditions.

As it was pointed out by Plant (1982, p. 1961), the role of nonlinearity in formation of wind-wave spectra is substantially underestimated.

\subsection{Experimental approximations for wind-wave spectra} JONSWAP spectrum

Similarity analysis is widely used in the wind-wave studies since 1962, when Kitaigorodskii proposed to use dimensionality analysis to construct a shorter set of non-dimensional arguments of wind-wave spectra. In the simplest case of deep water waves the dimensional variables are: wave frequency $\omega$, gravity acceleration $g$, friction velocity $u_{*}$ (or wind speed at some height), time $t$ and fetch $x$. The wind-wave frequency spectra (Sect. 2.3) are written in non-dimensional variables as follows

$$
\frac{E(\omega, t, x) g^{3}}{u_{*}^{5}}=F\left(\omega u_{*} / g, g t / u_{*}, g x /\left(u^{*}\right)^{2}\right)
$$

Here $F\left(\omega u_{*} / g, g t / u_{*}, g x /\left(u^{*}\right)^{2}\right)$ is a non-dimensional function of non-dimensional frequency, time and fetch. Such characteristic scales of the spectra as the frequency of spectral peak $\omega_{p}$ and the wave height dispersion $\langle E\rangle^{1 / 2}$ are assumed to be functions of non-dimensional "external" variables only: $g t / u_{*}$ (time) or/and $g x /\left(u^{*}\right)^{2}$ (fetch). We should stress that almost in all experimental parameterizations of wind-wave spectra the dependencies on "external" parameters containing explicitly time or fetch are replaced by an "internal" parameter - the spectral peak frequency $\omega_{p}$. In such formulation the wind-wave spectra cease to depend explicitly on "external" time or fetch. As an example let us consider the JONSWAP spectrum (Hasselmann et al., 1973) that summarized experimental measurements of wind waves in the Northern Sea

$$
\begin{aligned}
E(\omega) & =\alpha_{J} g^{2} \omega^{-5} \exp \left[-\frac{5}{4}\left(\frac{\omega}{\omega_{p}}\right)^{-4}\right] \\
& \times \exp \left\{\ln \gamma \cdot \exp \left[-\frac{\left(\omega-\omega_{p}\right)^{2}}{2 \sigma_{p}^{2} \omega_{p}^{2}}\right]\right\}
\end{aligned}
$$

This formula is based on measurements that were carried out for the case of fetch-limited growth of waves. At the same 
time (Eq. 36) does not contain the dependence on fetch explicitly. The JONSWAP spectrum was proposed as a generalization of the Pierson and Moskowitz (1964) spectrum for the case of developing wind-wave field. Parameter $\omega_{p}$ is directly connected with the wave age $c=g /\left(\omega_{p} U_{10}\right)\left(U_{10} \approx u^{*} / 28\right.$ is the wind velocity at standard anemometry height $10 \mathrm{~m}$ ). Parameter $\alpha_{J}$ also depends on the wave age, contrary to the constant Phillips' parameter $\alpha_{P M} \approx 1.17 \times 10^{-2}$ and fixed characteristic frequency $\omega_{P M}=g / U_{20}$ of the PiersonMoskowitz spectrum $\left(U_{20}\right.$ is the wind speed at $20 \mathrm{~m}$ height). Parameters $\gamma$ and $\sigma_{p}$ are introduced to describe an important feature of the developing wind-wave spectra - their pronounced peakedness. These parameters are assumed to be dependent on wave age $c$.

Formally one can consider spectral parameterizations (Eq. 36) irrespective of the method of wind wave measurements. Additional arguments for unique parameterizations of fetch- and duration-limited growth data can be found in paper by Kahma (1981), where correlations of measured parameters with the fetch were analyzed.

A modified version of parameterizations in the same fetchfree form was proposed for the same JONSWAP data after re-analysis (Donelan et al., 1985; Battjes et al., 1987). The modified JONSWAP spectrum is written as

$$
\begin{aligned}
E(\omega) & =\alpha_{T} g^{2} \omega^{-4} \omega_{p}^{-1} \exp \left[-\left(\frac{\omega}{\omega_{p}}\right)^{-4}\right] \\
& \times \exp \left\{\ln \gamma \cdot \exp \left[-\frac{\left(\omega-\omega_{p}\right)^{2}}{2 \sigma_{p}^{2} \omega_{p}^{2}}\right]\right\}
\end{aligned}
$$

that keeps the high frequency tail asymptotics $\omega^{-4}$ in full accordance with predictions of weak turbulence theory (Zakharov and Filonenko, 1966). It should be stressed that the spectrum (Eq. 37) has been proposed with no influence of the theoretical work, basing on experimental facts only.

The total energy parameter $\alpha_{T}$ differs from $\alpha_{J}$ in Eq. (36). The standard shape parameters are

$$
\begin{gathered}
\gamma=3.3 \\
\sigma=\left\{\begin{array}{l}
\sigma_{a}=0.07 \quad \text { for } \quad \omega \leq \omega_{p} \\
\sigma_{b}=0.09 \quad \text { for } \quad \omega>\omega_{p}
\end{array}\right.
\end{gathered}
$$

Here $\omega_{p}$ is the characteristic frequency, i.e. an "internal" parameter of the wind-wave field. Parameter $\alpha_{T}$ depends on "external" parameters. This dependence is expressed in terms of non-dimensional parameter, the wave age $C_{p} / U_{10}$, so does not contain dependence on time and fetch explicitly. Parameters $\gamma, \sigma_{p}$, strictly speaking, depend on "external" parameters and they are fetch-free (duration-free) functions of the wave age as well (Babanin and Soloviev, 1998). In this paper we assume parameters $\gamma$ and $\sigma_{p}$ to be constant (see Eq. 38) when comparing with numerical results.

The dependence of parameter $\alpha_{T}$ on the inverse wave age $U_{10} / C_{p}=\omega_{p} U_{10} / g$ is generally fitted by power-like approximation (Young, 1999)

$\alpha_{T}=\alpha_{0}\left(U_{10} \omega_{p} / g\right)^{\kappa_{\alpha}}$
For $\alpha_{0}$ we accept the following estimate (Eq. 25 in Babanin and Soloviev, 1998):

$\alpha_{0}=0.08 /(2 \pi)$

The exponent $\kappa_{\alpha}$ can vary in rather wide range depending on wind conditions.

Emphasize two features of almost all wind wave spectra parameterizations including Eqs. (36) and (37):

- There are two non-dimensional arguments: the wave frequency $\omega / \omega_{p}$ ("internal" argument) and the wave age ("external" argument);

- The dependence of wind-wave spectra on these two arguments is split.

The JONSWAP parameterization (Eqs. 37 and 39) yields:

$$
\begin{aligned}
E(\omega) & =\frac{\alpha_{0} g^{2}}{\omega_{p}^{5}} F_{n l}\left(\frac{\omega}{\omega_{p}}\right) F_{\text {ext }}\left(\frac{U_{10} \omega_{p}}{g}\right) \\
& =\frac{\alpha_{0} g^{2}}{\omega_{p}^{5}}\left(\frac{U_{10} \omega_{p}}{g}\right)^{\kappa_{\alpha}} F_{n l}\left(\frac{\omega}{\omega_{p}}\right)
\end{aligned}
$$

where

$F_{n l}(x)=x^{-4} \exp \left(-x^{-4}\right) \times \exp \left\{\ln \gamma \cdot\left[-\frac{(x-1)^{2}}{2 \sigma_{p}^{2}}\right]\right\}$

is the universal function of $x=\omega / \omega_{p}$. This function has a freakish form, while its counterpart $F_{\text {ext }}$ is a power-like function of the "external" wave age argument.

Integrating Eq. (41) over frequency one has

$\frac{E_{t o t} \omega_{p}^{4}}{g^{2}}=\alpha_{0}\left(\frac{U_{10}}{C_{p}}\right)^{\kappa_{\alpha}} F_{t o t}$

Putting $\kappa_{\alpha}=1$ into Eq. (42) we get exactly the Toba's law (Toba, 1997) in terms of significant wave height $a_{s}$ and mean wave period $T_{s}$ :

$a_{s}=B\left(g u_{*}\right)^{1 / 2} T_{s}^{3 / 2}$

The Toba's constant

$B=\left(2 \pi \alpha_{0} F_{t o t} U_{10} / u_{*}\right)^{1 / 2} /(2 \pi)^{2}$

can be easily calculated assuming $U_{10} \approx 28 u_{*}$.

For $\alpha_{0}=0.08 / 2 \pi$ (Babanin and Soloviev, 1998) one has

$B=0.095\left(F_{\text {tot }}\right)^{1 / 2}=0.0632$

that is very close to Toba's value $B=0.062$ (Toba, 1973).

We must stress again that Eq. (41) describes the typical case of "incomplete" self-similarity, when the spectrum shape depends on internal self-similar argument $x=\omega / \omega_{p}$ in very complicated way but dependence on key external parameter - wave age - has essentially asymptotic nature of a monotonic power-like dependence. As new experimental data become available, the self-similarity of wind wave spectra and the universality of spectra parameterizations are discussed more and more widely (see Babanin and Soloviev, 1998). We use the idea of self-similarity of wind-wave spectra as a basis for comparison of our numerical results with experimental data. 
3.2 Conventional parameterizations of wind-wave input term $S_{\text {in }}$

A number of parameterizations is proposed for the wave input term $S_{i n}$. They are based on the simplest physical models and on results of experimental studies. Generally, all parameterizations imply linearity or quasi-linearity of the wave input

$S_{\text {in }}=\beta(\boldsymbol{k}) N(\boldsymbol{k})$

Growth rate $\beta(\boldsymbol{k})$ is usually related to a resonant mechanism of surface wave generation, i.e. it takes a form

$\beta(\boldsymbol{k})=\varrho \omega(\boldsymbol{k}) F(\varsigma)$

where small parameter of wave generation

$\varrho=\rho_{a} / \rho_{w}$

is a ratio of air and water densities. Resonant nature of wave generation is associated with the ratio of wind speed $U_{h}$ at some height $h$ (or its substitute friction velocity $u_{*}$ ) to the wave phase speed. The dependence of $\beta$ on angle is taken into account in a Cerenkov-like form as follows

$\varsigma=s \frac{U_{h}}{C_{p h}} \cos \theta$

Here $s$ is a coefficient close to 1 , angle $\theta$ is related to wind direction.

Let us describe the commonly used parameterizations of wave input that we will refer to later in the paper.

Snyder et al. (1981) proposed the following formula for the wind wave generation rate

$\beta=\left\{\begin{array}{lc}(0.2 \div 0.3) \frac{\rho_{a}}{\rho_{w}} \omega(\varsigma-1), & s=1, \quad 1<\varsigma<3 \\ 0, & \text { otherwise }\end{array}\right.$

Here $U_{h}$ is the wind speed at $5 \mathrm{~m}$ height. The rate $\beta$ is linear in $\varsigma$ near the low-frequency limit of the generation domain and likely overestimates the generation at peak frequency for the developing wave field. This formula is obtained for relatively narrow wave frequency band $1<\varsigma<3$ while in many wave models these restrictions are completely ignored. For $\varsigma \rightarrow \infty$ the non-dimensional increment $\beta / \omega$ grows linearly.

Plant (1982) proposed stronger growth of $\beta$ with frequency

$\beta=\left\{\begin{array}{l}(0.04 \pm 0.02) \omega\left(\frac{u_{*}}{C_{p h}}\right)^{2} \cos \theta, \\ \text { for } \frac{g}{2 \pi U_{10}}<\frac{\omega}{2 \pi}<20 \mathrm{~Hz}, \quad \cos \theta>0 \\ 0, \quad \text { otherwise }\end{array}\right.$

or in terms of $U_{10}\left(u_{*}^{2} / U_{10}^{2} \approx \rho_{a} / \rho_{w}\right)$

$\beta=\left\{\begin{array}{l}(0.04 \pm 0.02) \frac{\rho_{a}}{\rho_{w}} \omega\left(\frac{U_{10}}{C_{p h}}\right)^{2} \cos \theta, \\ \text { for } \frac{g}{2 \pi U_{10}}<\frac{\omega}{2 \pi}<20 \mathrm{~Hz}, \quad \cos \theta>0 \\ 0, \quad \text { otherwise }\end{array}\right.$
In Eq. (49) the non-dimensional growth rate $\beta / \omega$ depends quadratically on wave frequency. Plant emphasizes that this behavior is consistent with experimental results at high frequencies, while at the low-frequency cut-off the increment does not vanish but has a finite magnitude.

Then Plant (1982) refers to Stewart's (1974) theoretical model, where the wave generation rate vanishes at lowfrequency cut-off

$\beta=0.04 \frac{\rho_{a}}{\rho_{w}} \omega \frac{U_{h}}{C_{p}}(\varsigma-1)$

Plant (1982) stresses the agreement of Eqs. (50) and (48) in the high frequency limit, while near the low-frequency cutoff their behavior is essentially different. $\beta / \omega$ in Eq. (50) grows linearly in $(\varsigma-1)$ as Snyder's formula (Eq. 47) predicts.

Hsiao and Shemdin (1983) proposed the following parameterization based on their own experimental data

$\beta=\left\{\begin{array}{lc}0.12 \frac{\rho_{a}}{\rho_{w}} \omega(\varsigma-1)^{2}, & s=0.85, \quad 1<\varsigma<7.4 \\ 0, & \text { otherwise }\end{array}\right.$

The wind speed $U_{h}$ is taken at $10 \mathrm{~m}$ height. Two features of Eq. (51) are important: $\beta$ vanishes quadratically at the low-frequency end and the maximal phase speed of amplified waves is lower than the wind speed. This is consistent with idea of vanishingly small generation near the spectral peak (Plant, 1982), or even of a rather strong wave damping in the peak vicinity (Hasselmann and Hasselmann, 1985). Equation (51) is justified for a wider frequency band as compared to Eq. (47). At the same time, authors accentuate the problem of high-frequency cut-off for the wind-wave increment $\beta$.

Donelan and Pierson-jr. (1987) summarized previous attempts to parameterize the wind wave input and came with the following formula

$\beta= \begin{cases}0.194 \frac{\rho_{a}}{\rho_{w}} \omega(\varsigma-1)^{2}, & s=1, \quad \varsigma>1 \\ 0, & \text { otherwise }\end{cases}$

Here the high-frequency limit is not specified explicitly and $U_{h}$ is taken at one-half of wavelength, $U_{h}=U_{\lambda_{w} / 2}$. Except the particular multipliers Eq. (52) is identical to Hsiao and Shemdin formula (Eq. 51).

Thus, we have in total five different models for $S_{i n}$. These models differ dramatically from each other. Except Snyder et al. (1981) they predict for short waves growth rates

$\beta / \omega \rightarrow \beta_{\infty} \frac{\rho_{a}}{\rho_{w}}\left(\frac{\omega U_{10}}{g}\right)^{2}$ at $\omega \gg g / U_{10}$

The same behavior of $\beta$ is predicted by Miles theory. However, different authors of the experimental formulas offer quite different values of $\beta_{\infty}$ as it is seen in Table 1 . The lowest value (Plant's and Stewart's models) is 5 times less than the highest one (Donelan's model). The distinction near the low-frequency cut-off is also dramatic: the parameterization by Plant (1982) predicts a finite growth rate $\beta$ at $\varsigma \rightarrow 1$, 
Table 1. Multipliers in high-frequency asymptotics (Eq. 53) for different parameterizations of wind-wave growth rates.

\begin{tabular}{ccccc}
\hline Model & Plant (1982) & Stewart (1974) & Hsiao and Shemdin (1983) & Donelan and Pierson-jr. (1987) \\
\hline$\beta_{\infty}$ & 0.04 & 0.04 & 0.087 & 0.194
\end{tabular}

formulas by Snyder et al. (1981) and Stewart (1974) are linear in $(\varsigma-1)$ while Donelan and Pierson-jr. (1987) and Hsiao and Shemdin (1983) dependencies are quadratic in $(\varsigma-1)$.

Figure 1 presents $\beta(\omega)$ for different models plotted in linear and in logarithmic scales and shows how limited our knowledge of $\beta$ is.

\subsection{Wave dissipation term $S_{\text {diss }}$ - "white-capping" mecha-} nism

All existing forecasting models (WAM, SWAN) include strong dissipation term $S_{\text {diss }}$, which is comparable with $S_{i n}$ and may surpass $S_{n l}$. However, physical mechanisms of wave energy dissipation are not studied properly: actually we know about $S_{\text {diss }}$ much less than about $S_{i n}$.

Certainly dissipation is essentially nonlinear process. Waves shorter than $1 \mathrm{~m}$ generate trains of gravity-capillary forced harmonics (Longuet-Higgins, 1995, 1996). Due to these harmonics the energy transfers to capillary waves, then the capillary waves dissipate due to viscosity. For the "smooth sea" in absence of white-capping this process is the leading mechanism of dissipation. In typical conditions the sea is "smooth" if the wind speed $U_{10}<5 \div 6 \mathrm{~m} \cdot \mathrm{s}^{-1}$. For stronger winds the generation of gravity-capillary slave harmonics turns to micro-breaking - the formation of quasiperiodic patterns of breakers with periods much less than ones of energy-containing waves (see, for example, Melville, 1996).

These mechanisms take away the energy from the highfrequency range of wave spectrum. Does there exist any mechanism of absorbtion of energy from the spectral peak range? From the physical view-point the answer to this question is unclear. Usually the mechanism of energy dissipation is associated with white-capping. The white-capping appears if the wind velocity is higher than $5 \div 6 \mathrm{~m} \cdot \mathrm{s}^{-1}$. Density of white-capping grows fast with the wind velocity and at $U_{10}>15 \mathrm{~m} \cdot \mathrm{s}^{-1}$ each leading wave has a white cap. Certainly, the white caps present the area of energy dissipation. However, the assumption that this dissipation is also concentrated in high wave numbers is very natural. The white cap, persisting at the crest of a long energy-containing wave at strong wind, can be interpreted in a following way. There is some nonlinear mechanism that tries to form a wedge-type singularity on the surface and the white-capping smoothes this singularity. If this mechanism takes place on the crest of limiting Stokes wave as Phillips (1958) assumed, it should take away the energy from the spectral peak. However, characteristic steepness of the leading wave with $\mu \leq 0.1$ is much less than the steepness of limiting Stokes wave $(\mu>0.4)$. For waves of such small steepness the short and long waves are not connected directly and dissipation of short waves does not lead immediately to dissipation of long waves.

Another argument against the white-capping mechanism for the long-wave dissipation is the structure of turbulence. The wave-induced turbulence is concentrated in a thin boundary layer beneath the surface. There are no traces of long vortices that should appear due to dissipation of long waves. In fact, the only visible argument in support of white-capping mechanism for long-wave dissipation is phenomenon of "sea maturity". It is considered that the spectral downshift is arrested when the spectral peak frequency $\omega_{p}$ is somewhat below the characteristic frequency $\omega_{0}=g / U_{10}$.

The concept of "mature sea" was offered by Pierson and Moskowitz (1964). Since that time this concept is still a subject of discussions. Some authors (Glazman, 1994), by remote sensing of ocean, reported observations of very long waves with phase velocities several times higher than wind speed. However, most authors agree that the maturing of the sea is a real phenomenon that takes place at very high fetches (of order $10^{4}$ wave lengths). For wave lengths $\lambda \sim 100 \mathrm{~m}$ this yields $1000 \mathrm{~km}$. We must stress that very weak dissipation $\beta / \omega \sim 10^{-5}$ can provide this effect. Indeed, the origin of this dissipation could be white-capping but existence of this dissipation does not mean that this is essential for shorter fetches which are more interesting from the practical view-point.

We will not discuss in this paper all empirical models for $S_{d i s s}$. We will mention two most popular ones only. The first model, offered by Hasselmann (1974) and widely used since that time is the following:

$S_{\text {diss }}=-C_{f} \bar{\omega}\left(\frac{\omega}{\bar{\omega}}\right)^{2}\left(\frac{\bar{\alpha}}{\bar{\alpha}_{P M}}\right)^{2} N(\boldsymbol{k})$

Here $C_{f}=3.33 \times 10^{-5}$ and $\bar{\alpha}_{P M}=4.57 \times 10^{-3}$ is the theoretical "Pierson-Moskowitz steepness" and both the mean frequency $\bar{\omega}$ and the non-dimensional energy $\bar{\alpha}$

$\bar{\alpha}=\frac{E_{t o t} \bar{\omega}^{4}}{g^{2}}$

depend on the wave field state. The ratio $\bar{\alpha} / \bar{\alpha}_{P M}$ in Eq. (54) can be estimated easily for conventional parameterizations of wind-wave spectra (e.g. JONSWAP, Hasselmann et al., 1973) using their property of self-similarity. While these parameterizations split the dependence of wave spectra on internal and external parameters - the non-dimensional wave frequency and the wave age - the mean values $\bar{\omega}$ and the nondimensional energy $\bar{\alpha}$ can be expressed in terms of wave age $g /\left(U_{10} \omega_{p}\right)$. One gets (see Eqs. 39 and 42 )

$$
\frac{\bar{\alpha}}{\bar{\alpha}_{P M}} \sim\left(\frac{U_{10} \omega_{p}}{g}\right)^{\kappa_{\alpha}}
$$



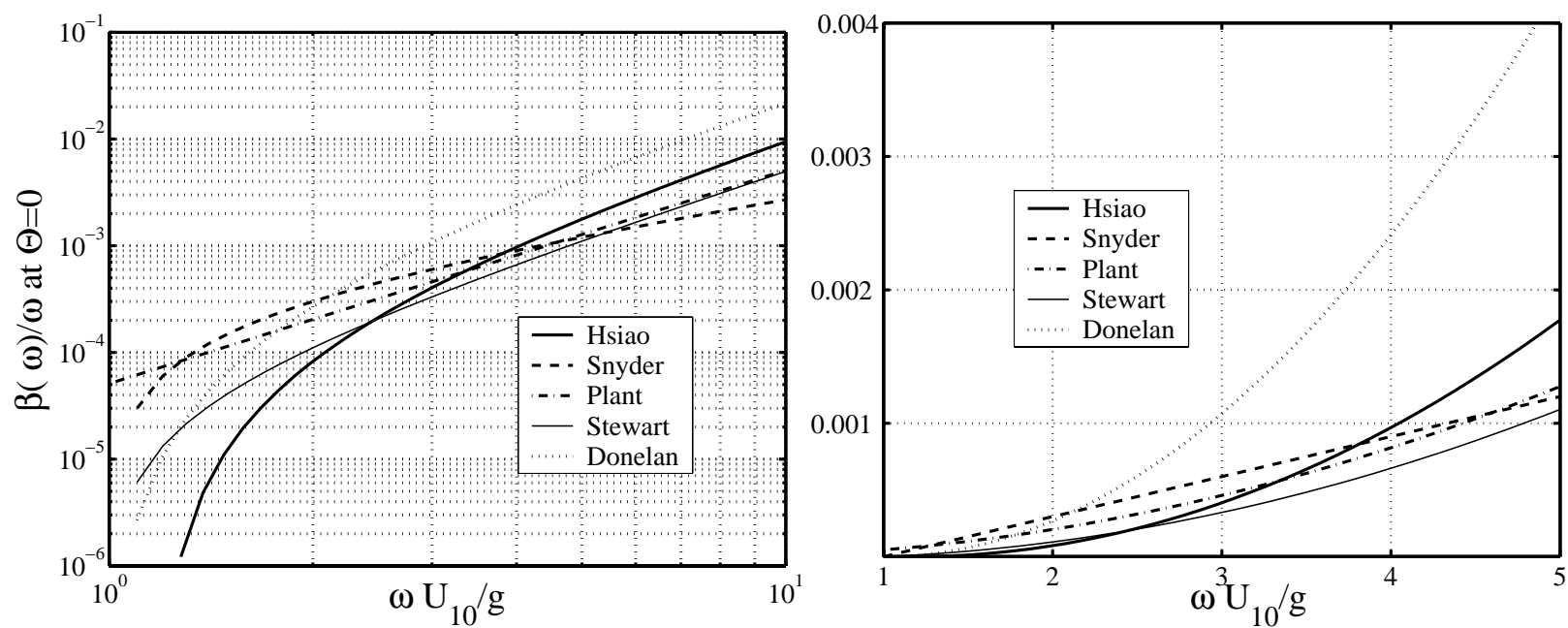

Fig. 1. Dependence of wind-wave growth rate on non-dimensional frequency $\omega U_{10} / g$ in log-and linear scales given by different experimental parameterizations (see legends).

and, finally, a fairly simple expression for the dissipation rate

$\frac{\beta_{d i s s}}{\omega}=-C_{\beta}\left(\frac{\omega U_{10}}{g}\right) \times\left(\frac{\omega_{p} U_{10}}{g}\right)^{2 \kappa_{\alpha}-1}$

Constant $C_{\beta}$ is close to the multiplier $3.33 \times 10^{-5}$ in Eq. (54). Toba's law for developing sea corresponds to $\kappa_{\alpha}=1$. That gives the dissipation rate in Eq. (56) growing with frequency as $\omega^{2}$ similarly to the generation rate by Snyder et al. (1981). Note, that the wave input rates in other models of the previous section grow faster, as $\omega^{3}$. The result looks paradoxically: the term $S_{\text {diss }}$ (Eq. 54) cannot balance the wave input in small scales, where the dissipation due to wave breaking must dominate.

In fact, in wave forecasting models the high frequency dissipation is introduced in implicit form in order to achieve numerical stability in small scales (Tolman, 1992) or it is introduced explicitly to keep correct quasi-stationary asymptotics of wave spectra in high-frequencies (e.g. Tolman and Chalikov, 1996). In the latter case the relative contribution of $S_{n l}, S_{i n}$ and $S_{d i s s}$ into the balance becomes a key problem.

Results of numerical experiments with mature wind waves (Komen et al., 1984) are considered usually as a justification of the Hasselmann white-capping mechanism (Hasselmann, 1974). Komen et al. (1984) calculated wave input $S_{\text {in }}$ and collision integral $S_{n l}$ for the Pierson-Moskowitz spectrum and found the dissipation term $S_{\text {diss }}$ as a residual one to provide a balance in the kinetic equation. All the three terms of wind-wave balance appeared to be close to each other in magnitudes near the spectral peak. Additionally, it was found that the dissipation can be parameterized surprisingly well by the white-capping formula (54) with slightly smaller coefficient $C_{f}$. In fact, the authors do not consider these results as a justification of the leading role of whitecapping mechanism in wind-wave evolution. They stress that their consideration is "... based on extrapolation ..." of experimental parameterizations obtained for developing windwave sea at rather weak winds $\left(4-6 \mathrm{~m} \cdot \mathrm{s}^{-1}\right.$ in experiments of Snyder et al., 1981) on the case of mature sea where so far there are no direct measurements of wave input and dissipation. Severe hypotheses underlying the analysis by Komen et al. (1984) were ignored by many followers. Unintentionally, this milestone paper became "a misguiding star": the problem of physical roots is replaced by the problem of tuning of wave dissipation to fit some superficial non-physical criteria.

This is seen in another model of $S_{\text {diss }}$, offered by Phillips (1985) and used by some authors in their theoretical constructions (see Hara and Belcher, 2002). The result

$S_{\text {diss }}=\alpha^{\prime \prime} \omega_{\boldsymbol{k}} N_{\boldsymbol{k}}\left(|\boldsymbol{k}|^{2} E_{\boldsymbol{k}}\right)^{2}$

( $\alpha^{\prime \prime}$ is a constant) coincides exactly with a simplistic estimate of $S_{n l}$ (Eq. 29). The form (Eq. 57) was taken deliberately to obtain the Zakharov-Filonenko spectrum $\omega^{-4}$ as a solution of the balance equation in the universal range. In fact, the spectrum $\omega^{-4}$ is a solution of equation $S_{n l}=0$ : to obtain this spectrum there are no reasons to include dissipation terms into consideration!

Some practical reason to include an artificial $S_{d i s s}$ term into the Hasselmann equation does exist. As we mentioned above, there is a big diversity of wind-input terms $S_{i n}$ and the results of numerical simulation of the Hasselmann equation depend essentially on the choice of $S_{i n}$. It is shown (Komen et al., 1984; Pushkarev et al., 2003) that if $S_{\text {in }}$ is taken in Eq. (47) proposed by Snyder et al. (1981) or Eq. (52) by Donelan and Pierson-jr. (1987) the waves grow too fast as compared to experimental data. In this case including $S_{\text {diss }}$ can fix the situation. However, this is not a "physical" argument. We will show that numerical simulations with less aggressive form of $S_{\text {in }}$ (Eqs. 51 and 48) by Hsiao and Shemdin 
(1983) or Plant (1982) make possible to obtain good agreement with experiment without using any artificial $S_{d i s s}$.

3.4 Nonlinearity vs. wave input and dissipation - direct comparison of the terms

The problem of dominating mechanisms of the wind-wave balance can be easily solved by numerical simulation of the Hasselmann equation. Comparison of terms in the kinetic equation does not require the solution of the evolution problem. It is enough to make "snapshots" of terms for different spectra. While the "snapshot" is unique for the "first principle term" $S_{n l}$, different forms of empirical dependence for $S_{n l}, S_{d i s s}$ can be used for the comparison.

\subsubsection{Comparison of different parameterizations for wave input}

Comparison of source terms for different parameterizations of wave growth rate (Eqs. 47-52) is given in Fig. 2. The JONSWAP forms of frequency spectra $E(\omega)$ (Donelan et al., 1985) for wind speed $U_{10}=10 \mathrm{~m} \cdot \mathrm{s}^{-1}$ and three different wave ages are taken for the comparison. To calculate the corresponding spatial spectrum the simplest form of angular dependence was chosen

$N(\boldsymbol{k})= \begin{cases}N(|\boldsymbol{k}|) \cos ^{2} \theta, & \text { for }-\pi / 2<\theta<\pi / 2 \\ 0, & \text { otherwise }\end{cases}$

In fact, the observed wind wave spectra have more complicated dependence on angle but the above form is sufficient to fix important problems of wind input parameterizations. The source terms are calculated as they appear in the kinetic Eq. (27) for wave action (left column in Fig. 2). In the right column the spectral density of the input term averaged in angle is shown.

Figures for three different wave ages show clearly a rather strong difference of wave input terms. For young waves (top row), all formulas show similar behavior and the agreement can be achieved by simple tuning of the corresponding multipliers. Parameterizations by Donelan and Pierson-jr. (1987); Plant (1982); Snyder et al. (1981) have close magnitudes while Hsiao and Shemdin (1983) and Stewart (1974) parameterizations form an alternative group with essentially lower values.

The difference of parameterizations for the wave input becomes dramatic for "old" waves: $\omega_{p} U_{10} / g=1$ (middle row) and $\omega_{p} U_{10} / g=0.9$ (bottom row). We emphasized this problem in Sect. 3.2: the parameterizations have perfectly different behavior (constant, linear or quadratic in $\left.\left(\varsigma^{-1}\right)\right)$ near the low-frequency cut-off of $\beta(\omega)$. Both magnitudes and forms of dependencies are essentially different. The annoying question of the comparison is: "What parameterizations should be used in the kinetic equation? Which formula is true?"

\subsubsection{Nonlinearity vs. wave input for JONSWAP spectra}

The similar term-to-term comparison can be performed for the collision integral $S_{n l}$ calculated for a "reference" spectrum JONSWAP. Results are presented in Fig. 3 for the same spectra as in the previous section and for inverse wave ages $\omega_{p} U_{10} / g=2$ and $\omega_{p} U_{10} / g=1.5$. The first point to be stressed is nonlinear transfer dominates at rather early stages of wind wave evolution.

In fact, this is true near the spectral peak, where $S_{n l}$ exceeds $S_{i n}$ significantly: approximately 4 times for $\omega_{p} U_{10} / g=2$ and 8 times for $\omega_{p} U_{10} / g=1.5$. In order to fix this difference definitely we show $S_{n l}$ (middle, right in Fig. 3) and $S_{f}=S_{i n}+S_{\text {diss }}$ (bottom, right in Fig. 3) in the same scales in Fig. 4.

Note the strong effect of spectra peakedness on $S_{n l}$ : for $\gamma=1$ (the Pierson-Moskowitz spectral form) the difference of $S_{n l}$ and $S_{i n}$ is noticeably less than for higher values of $\gamma$. In perfect agreement with results of Sect. 2, we see that peakedness amplifies the nonlinear transfer dramatically. Underestimation of peakedness effect is likely a source of misleading results on secondary role of nonlinear transfer in the kinetic equation (see discussion in Sect. 3.3).

In the inertial interval, comparison of different terms in the kinetic equation is not a trivial problem. In this spectral range when $S_{n l} \rightarrow 0$ and $S_{n l}+S_{f} \rightarrow 0$ a term-to-term comparison can be misleading. To find which term is more important one has to impose a small perturbation to the stationary solution

$N=N_{0}+\delta N$

and study the kinetic equation in variational form (Balk and Zakharov, 1988, 1998)

$$
\begin{aligned}
\frac{\partial \delta N(\boldsymbol{k})}{\partial t} & =\beta_{i n}(\boldsymbol{k}) \delta N\left(\boldsymbol{k}^{\prime}\right)+\beta_{\text {diss }}(\boldsymbol{k}) \delta N\left(\boldsymbol{k}^{\prime}\right) \\
& +\int R\left(\boldsymbol{k}, \boldsymbol{k}^{\prime}\right) \delta N\left(\boldsymbol{k}^{\prime}\right) d \boldsymbol{k}^{\prime}
\end{aligned}
$$

where

$R\left(\boldsymbol{k}, \boldsymbol{k}^{\prime}\right)=\frac{\delta S_{n l}(\boldsymbol{k})}{\delta N\left(\boldsymbol{k}^{\prime}\right)}$

is the Fréchet derivative of the collision integral. The effect of local (in wavevector space) terms of wave input and dissipation can be estimated as a characteristic time of exponential growth (decay) - the relaxation time

$\tau_{f}=\frac{1}{\beta_{\text {in }}+\beta_{\text {diss }}} ; \quad \beta_{\text {diss }}<0$

For the collision integral $S_{n l}$ a crude approximation of the similar time scale gives

$\tau_{n l}=\left(\int R\left(\boldsymbol{k}, \boldsymbol{k}^{\prime}\right) d \boldsymbol{k}^{\prime}\right)^{-1} \sim \frac{1}{\gamma_{\boldsymbol{k}}}$

Here $\gamma_{\boldsymbol{k}}$ is introduced by Eq. (31). It should be stressed that the relaxation time $\tau_{n l}$ depends essentially on the solution and this dependence is not local in wavevector space. Relaxation times were estimated numerically by Resio and Perrie 

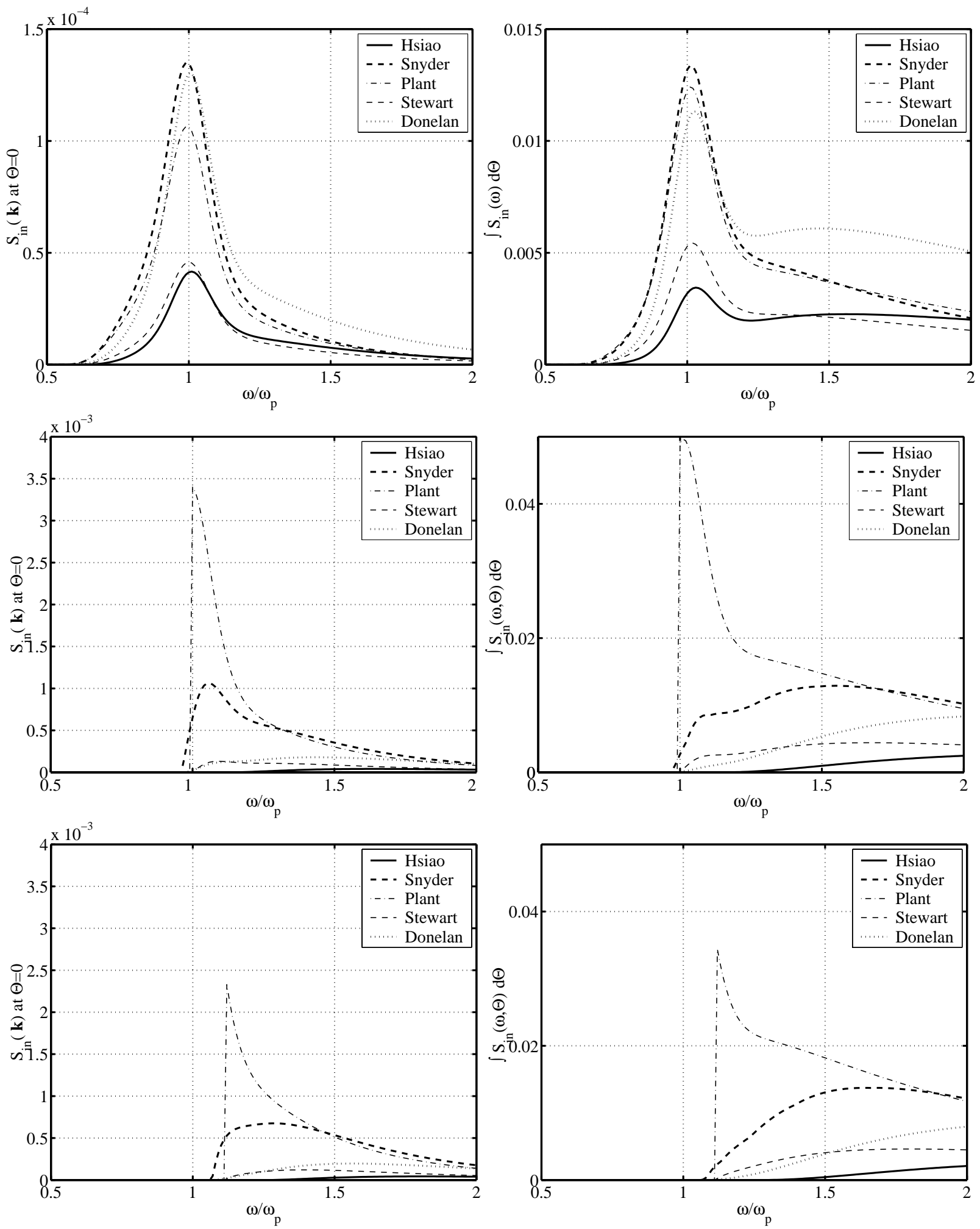

Fig. 2. Wave input functions as they appear in the right-hand side of the kinetic equation for wave action (left column) and the averaged one in angle (right column) for different wave ages and different parameterizations of wind input (shown in legends) $\left(U_{10} \omega_{p} / g=2-\right.$ top, $U_{10} \omega_{p} / g=1$ - center, $U_{10} \omega_{p} / g=0.9$ - bottom) as functions of non-dimensional wave frequency $\omega / \omega_{p}$. The JONSWAP spectrum with the standard set of parameters is taken for wind speed $U_{10}=10 \mathrm{~m} \cdot \mathrm{s}^{-1}$ (see Sect. 3.1). 

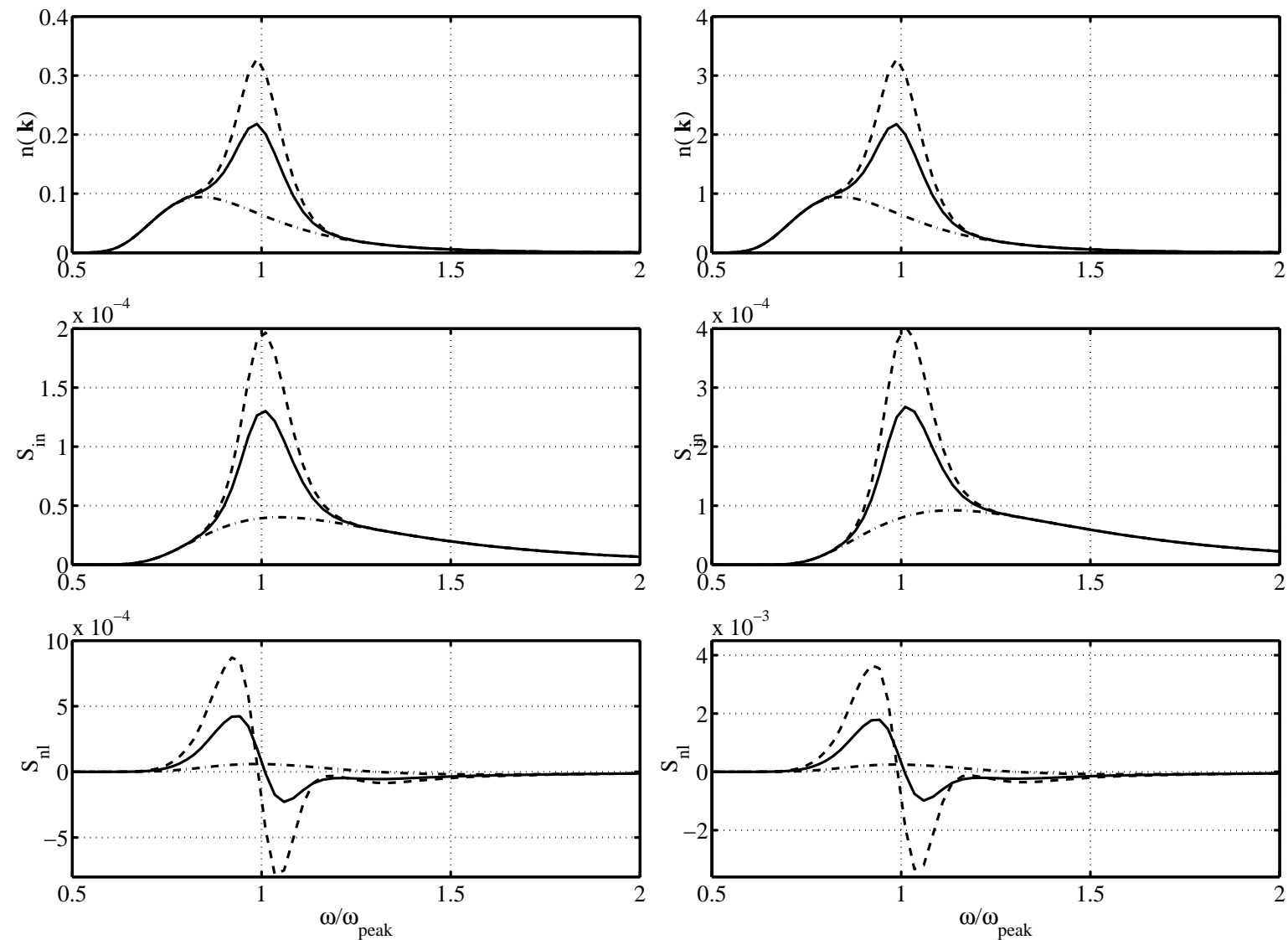

Fig. 3. Upper row - instantaneous JONSWAP spectra, middle row - wave input term $S_{\text {in }}$ by Donelan et al. (1987), bottom row - collision integral $S_{n l}$ as they appear in the kinetic Eq. (2) for down-wind direction. Wind speed $U_{10}=10 \mathrm{~m} \cdot \mathrm{s}^{-1}$, angular dependence of JONSWAP spectra is $\cos ^{2} \theta$. Left column shows results for inverse wave age $U_{10} \omega_{p} / g=2$ and right - for $U_{10} \omega_{p} / g=1.5$. Results for three values of peakedness parameter $\gamma=1,3.3,5$ are shown by different curves (dash-dot, solid, dashed, correspondingly). The abscise is non-dimensional wave frequency. Note, that the term scaling is different for the columns: the spectrum peak is approximately 10 times, the input term is 2 times and the collision integral is approximately 4 times higher for "older" waves $\left(U_{10} \omega_{p} / g=1.5\right.$ (right column). Collision integral amplitudes grow faster than the wave input term and exceed the term by factor 8 for the sharpest $(\gamma=5)$ JONSWAP spectrum at $U_{10} \omega_{p} / g=1.5$.

(1991, see Figs. 18 and 19). Their calculation gives rather short times in a range $10^{2}-10^{3} \mathrm{~s}$. We do not present similar estimates in this paper. The dominating role of $S_{n l}\left(\operatorname{short} \tau_{n l}\right.$ ) will be demonstrated as an inherent property of the resulting solutions - their tendency to self-similar behavior.

As we mentioned, the JONSWAP spectrum have selfsimilarity features. Together with re-scaling property of the collision integral (Eq. 29) it gives a simple dependence of $S_{n l}$ on wave age (see Eq. 37)

$S_{n l} \sim\left(\omega_{p} U_{10} / g\right)^{3 \kappa_{\alpha}-8}$

For $\kappa_{\alpha}=1$ (Toba's law) Eq. 59 gives $3 \kappa_{\alpha}-8=-5$, i.e. a very rapid growth of $S_{n l}$ with spectrum downshift. Figure 3 (bottom row) follows this scaling fairly well.

The input term $S_{i n}=\beta(\boldsymbol{k}) N(\boldsymbol{k})$ does not allow the selfsimilar re-scaling. For JONSWAP spectrum this term grows slower than $S_{n l}$ because the growth rate $\beta(\boldsymbol{k})$ sharply decreases when the wave phase speed is tending to the wind speed $U_{10}$. At the same time, the dissipation term $S_{\text {diss }}$ (Eq. 56) grows faster than the nonlinear transfer term

$S_{\text {diss }} \sim\left(\omega_{p} U_{10} / g\right)^{3 \kappa_{\alpha}-9}$

and, formally, can dominate for sufficiently old waves. However, this term appears to be small for reasonable wave ages $g /\left(\omega_{p} U_{10}\right)<1.5$ because of small multiplier in Eq. (54). The effect of wave spectra saturation due to the white-capping dissipation has been observed in numerical experiments (Komatsu and Masuda, 1996).

\subsubsection{Nonlinearity of "natural" spectra at early stages of} wave field evolution

As we will show below, the JONSWAP parameterization appears to be close to our numerical solutions for the kinetic equation. At the same time, this parameterization and the "natural" spectra obtained in our numerical experiments are not identical. The minor difference of the spectra are of 
no practical importance but this difference can affect significantly the magnitudes of the kinetic equation terms.

Figure 5 shows "snapshots" of the collision integral $S_{n l}$ and the input term $S_{i n}$ for the solutions of kinetic Eq. (2) for the same values of wave ages as in Fig. 3. The details of numerical approach will be given below. The point to be stressed here is that small difference in spectral forms can affect significantly our quantitative results. First, the peak values of both JONSWAP and numerical solutions appear to be very close to each other for the standard peakedness parameter $\gamma=3.3$. The minor difference is in the wave age definition: the peak of JONSWAP distribution is shifted slightly to lower frequencies as compared to the parameter $\omega_{p}$, while for "natural" spectra $\omega_{p}$ corresponds exactly to the spectral peak. This tiny mismatch leads to $20-30 \%$ excess in peak values of the input term $S_{i n}$ and $50 \%$ of growth of $S_{n l}$ as compared to the JONSWAP spectra.

As a summary of the section two points should be stressed. First, the collision integral in the kinetic equation starts to dominate at very early stages of wind wave evolution. We demonstrated this fact for the JONSWAP parameterization of wave spectra. Second, we showed that details of spectral distributions can affect the magnitude of the collision integral significantly. Thus, the question on contribution of different terms of the kinetic equation goes hand in hand with the question how these terms evolve within the kinetic equation. The comparison of "snapshots" of the terms for some "representative" parameterizations of wave spectra can be misleading as it was the case for the Pierson-Moskowitz spectrum (Komen et al., 1984, Sect. 3.3).

\section{Weak-turbulent Kolmogorov's spectra}

We presented preliminary arguments in favor of dominant role of nonlinear transfer in the Hasselmann equation. This statement implies applicability of basic results of weak turbulence theory for the case of wind-driven waves. The weak turbulence theory describes the transport of motion constants: energy, momentum and wave action along the spectrum. So far many members of the wind-wave community believe in "local balance" of energy in $\boldsymbol{k}$-space. According to this "local mentality", instability that causes exponential growth of wave in certain spectral range has to be arrested by nonlinear dissipative mechanism that takes place in the same spectral range. This concept is reasonable if nonlinear energy transport is a mechanism of secondary importance and its role is just to fix some mismatch between linear instability and nonlinear dissipation. However, if nonlinear interactions dominate such view-point is not tenable yet. Nonlinear interaction leads to migration of energy along different spectral ranges. Now, the energy balance is nonlocal: the energy being generated in one spectral range (say, $2 \div 3 \omega_{p}$ ) can be absorbed by some dissipative mechanisms (at $6 \div 7 \omega_{p}$ or so). The energy, momentum and wave-action transfer along the spectrum is described by the Kolmogorov (or KolmogorovZakharov) spectra, which play the central role in the theory

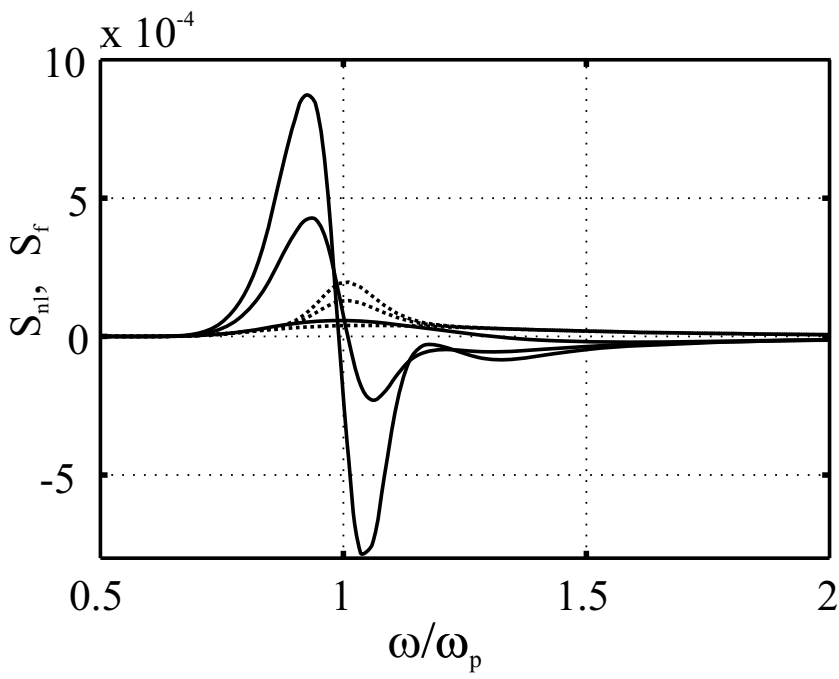

Fig. 4. Comparison of nonlinear transfer term $S_{n l}$ (hard line) and the term of forcing $S_{f}=S_{i n}+S_{\text {diss }}$ (dotted line) in the kinetic Eq. (2) for the case of left panel Fig. $3\left(U_{10} \omega_{p} / g=2\right)$ and different peakedness (see Fig. 3 caption).

of weak turbulence. The Kolmogorov spectra are exact solutions of stationary homogenous kinetic equation

$S_{n l}=0$

Basic features of the Kolmogorov solutions are reproduced in a number of numerical and experimental studies. The best known result of the theory - the exponent (-4) for highfrequency tail of wind-wave spectra - is in perfect agreement with available experimental data. The same spectrum is systematically observed in numerical experiments. However, the majority of wind-wave community does not associate so far this spectrum with the weak-turbulent theory. Meanwhile, this is the simplest weak-turbulent Kolmogorov spectrum. This spectrum was found analytically by Zakharov and Filonenko (1966), in 1972 this spectrum was observed experimentally by Toba (1973). Since that time the $\omega^{-4}$-spectrum was observed many times in the ocean, in laboratory and in numerical experiments. This spectrum describes the direct cascade of energy that migrates from small to high wavenumbers.

An opposite tendency - migration of wave action from infinitely small to large scales is presented by the inverse cascade solution that gives close exponent $11 / 3$ for frequency spectra. This solution was found by Zakharov (1966) in sixties, in seventieth it was studied as a particular case of non-equilibrium stationary distributions of particles (quasiparticles) in a number of physical problems (Katz and Kontorovich, 1971, 1974; Katz et al., 1975). Just in eighties the inverse cascade solution has been related to the problem of wind-wave spectra evolution (Zakharov and Zaslavskii, 1982; Zakharov and Zaslavsky, 1983), in particular, to the well-known phenomenon of wave spectra downshift.

In this section we give basic results of the theory of the Kolmogorov-Zakharov cascade solutions. 

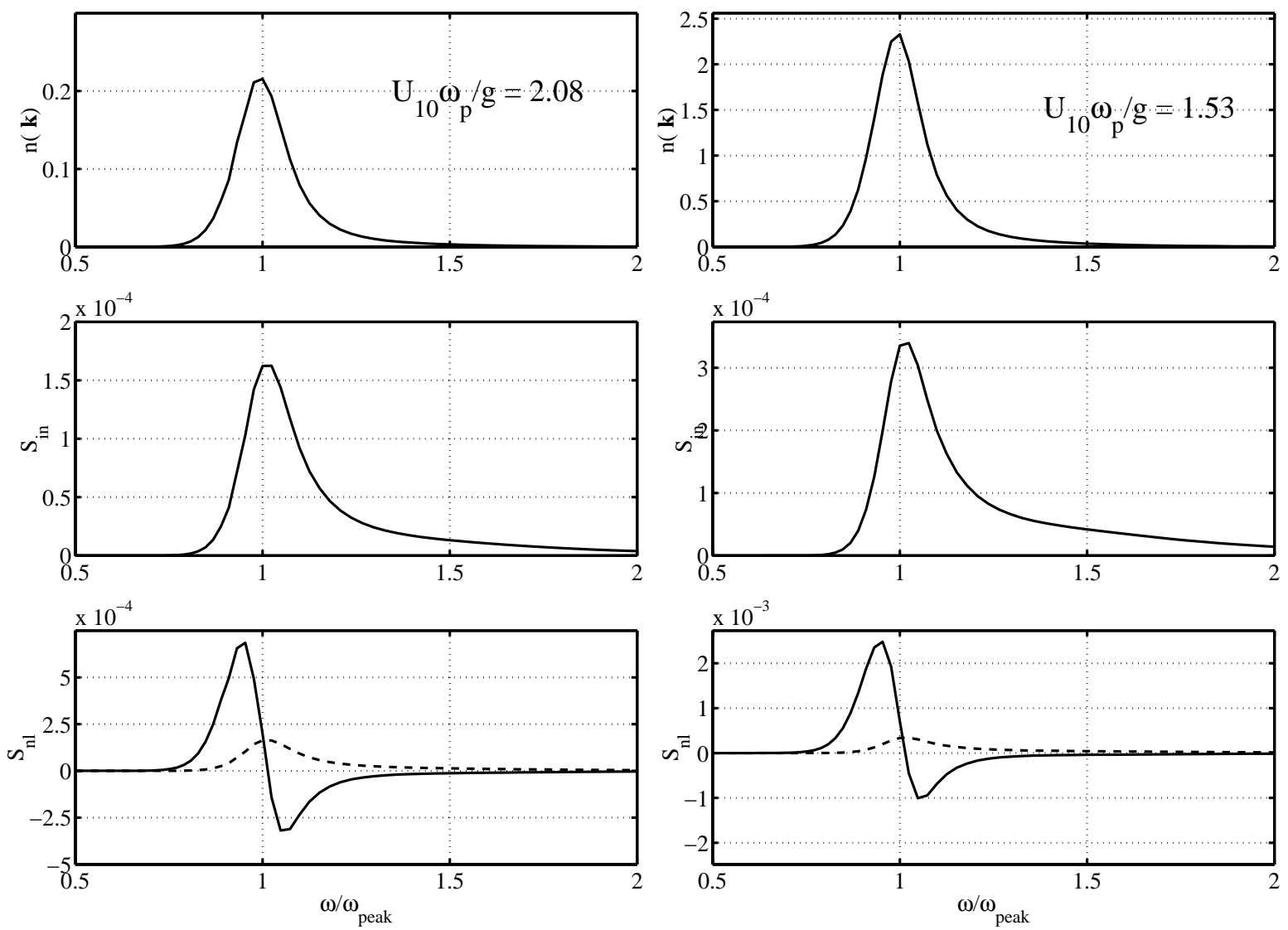

Fig. 5. The same as in Fig.3 for spectra resulting from evolution within the Hasselmann equation. The wave input term $S_{i n}$ is given twice: in middle (solid line) and in bottom row (dashed) for direct comparison with magnitudes of the collision integral $S_{n l}$.

\subsection{Definitions of constants of motion and their fluxes}

Let us consider basic properties of conservative homogeneous kinetic equation

$\frac{\partial N(\boldsymbol{k}, t)}{\partial t}=S_{n l}$

i.e. Eq. (27) in absence of wind forcing, dissipation and spatial inhomogeneity. The following quantities are constants of motion for Eq. (61)

$$
\begin{aligned}
N & =\int N(\boldsymbol{k}, t) d \boldsymbol{k}=\int N(\omega, t) d \omega \\
E & =\int \omega(\boldsymbol{k}) N(\boldsymbol{k}, t) d \boldsymbol{k}=\int \omega N(\omega, t) d \omega \\
\boldsymbol{M} & =\int \boldsymbol{k} N(\boldsymbol{k}, t) d \boldsymbol{k}=\int \boldsymbol{k} N(\omega, t) d \omega
\end{aligned}
$$

Let us formulate this statement more accurately. Actually, conservation of motion constants means that the following differential relations are valid

$$
\begin{gathered}
\frac{\partial N(\omega, t)}{\partial t}=\frac{\partial Q(\omega, t)}{\partial \omega} \\
\frac{\partial E(\omega, t)}{\partial t}=-\frac{\partial P(\omega, t)}{\partial \omega}
\end{gathered}
$$

$$
\frac{\partial \boldsymbol{M}(\omega, t)}{\partial t}=-\frac{\partial \boldsymbol{K}(\omega, t)}{\partial \omega}
$$

where the corresponding fluxes of wave action, energy and momentum production are defined as follows

$$
\begin{gathered}
Q(\omega)=\int_{\omega_{l}}^{\omega} \int_{-\pi}^{\pi} \frac{2 \omega^{3}}{g^{2}} S_{n l}(\boldsymbol{k}) d \omega d \theta \\
P(\omega)=-\int_{\omega_{l}}^{\omega} \int_{-\pi}^{\pi} \frac{2 \omega^{4}}{g^{2}} S_{n l}(\boldsymbol{k}) d \omega d \theta \\
K_{x}(\omega)=-\int_{\omega_{l}}^{\omega} \int_{-\pi}^{\pi} \frac{2 \omega^{5}}{g^{3}} S_{n l}(\boldsymbol{k}) \cos \theta d \omega d \theta
\end{gathered}
$$

We assume that momentum $\boldsymbol{M}$ and its flux $\boldsymbol{K}$ have only one component aligned along the x-axis. Positive signature of $Q$ corresponds to wave action flux to small wavenumbers, while positive fluxes of energy $P$ and momentum $K_{x}$ are directed to high wavenumbers.

It is useful to present the "flux form" of the kinetic equation in polar coordinates as

$\frac{\partial N(\omega, \theta, t)}{\partial t}=L A$

where

$L=\frac{1}{2} \frac{\partial^{2}}{\partial \omega^{2}}+\frac{1}{\omega^{2}} \frac{\partial^{2}}{\partial \theta^{2}}$ 
and the auxiliary function $A$ can be found as a result of action of nonlinear integral operator on $N(\omega, \theta)$

$$
\begin{aligned}
A(\omega, \theta) & =\int F\left(\omega, \omega_{1}, \omega_{2}, \omega_{3}, \theta-\theta_{1}, \theta-\theta_{2}, \theta-\theta_{3}\right) \\
& \times N\left(\omega_{1}, \theta_{1}\right) N\left(\omega_{2}, \theta_{2}\right) N\left(\omega_{3}, \theta_{3}\right) \\
& \times d \omega_{1} d \omega_{2} d \omega_{3} d \theta_{1} d \theta_{2} d \theta_{3}
\end{aligned}
$$

The explicit expression of $F$ is given in Appendix B. An additional auxiliary function can be introduced in a similar way for the "flux form" of the kinetic Eq. (2) rewritten for energy spectral density

$$
\begin{aligned}
& B(\omega, \theta)=\int \omega F\left(\omega, \omega_{1}, \omega_{2}, \omega_{3}, \theta-\theta_{1}, \theta-\theta_{2}, \theta-\theta_{3}\right) \\
& \times N\left(\omega_{1}, \theta_{1}\right) N\left(\omega_{2}, \theta_{2}\right) N\left(\omega_{3}, \theta_{3}\right) d \omega_{1} d \omega_{2} d \omega_{3} d \theta_{1} d \theta_{2} d \theta_{3}
\end{aligned}
$$

For angular mean values one gets

$$
\begin{aligned}
& A(\omega)=\frac{1}{2 \pi} \int_{0}^{2 \pi} A(\omega, \theta) d \theta \\
& B(\omega)=\frac{1}{2 \pi} \int_{0}^{2 \pi} B(\omega, \theta) \cos \theta d \theta
\end{aligned}
$$

Useful relation between energy, wave action and momentum fluxes can be obtained for spectral fluxes (Eqs. 62-64)

$$
\begin{aligned}
Q(\omega) & =\frac{\partial A}{\partial \omega} \\
P(\omega) & =A-\omega \frac{\partial A}{\partial \omega} \\
K_{x}(\omega) & =\frac{\omega}{g}\left(2 B-\omega \frac{\partial B}{\partial \omega}\right)
\end{aligned}
$$

\subsection{Kolmogorov's solutions}

Finding solutions for the Hasselmann kinetic equation is the subject of the theory of weak turbulence. The main point of this theory is investigation of the stationary Eq. (60).

\subsubsection{Local balance solutions}

Thermodynamic spectra correspond to local in wavevector (frequency) space balance. In this case nonlinear interactions vanish for each quartet of interacting waves. In other words, amplitudes of an interacting quartet

$N_{0} N_{1} N_{2}+N_{0} N_{1} N_{3}-N_{0} N_{2} N_{3}-N_{1} N_{2} N_{3}=0$

are balanced at every point of resonant surface

$$
\left\{\begin{array}{l}
\boldsymbol{k}_{0}+\boldsymbol{k}_{1}=\boldsymbol{k}_{2}+\boldsymbol{k}_{3} \\
\omega_{0}+\omega_{1}=\omega_{2}+\omega_{3}
\end{array}\right.
$$

This balance does not depend on interaction kernels that results in thermodynamic solution

$N(\boldsymbol{k})=\frac{T}{\omega_{k}+\mu}$

where temperature $T$ and $\mu$ are arbitrary parameters. This Rayleigh-Jeans solution appears in a great number of physical problems. However, this solution is completely irrelevant to the problems of wind-driven sea because the corresponding energy spectrum does not decay at $|\boldsymbol{k}| \rightarrow \infty$.

\subsubsection{Constant flux Kolmogorov's solutions}

Stationary kinetic Eq. (60) has a vast family of exact solutions completely different from the Rayleigh-Jeans spectrum (Eq. 69). These solutions were found by Zakharov with co-authors (Zakharov and Filonenko, 1966; Zakharov, 1966; Zakharov and Zaslavskii, 1982). In these articles authors used the so-called Zakharov conformal mapping to construct the solutions (see also Katz and Kontorovich, 1971; Katz et al., 1976). For the same purpose much more simple technique was offered in (Zakharov, 1999). The stationary kinetic Eq. (60) is equivalent to

$L A=0$

(see Eq. 65) that can by integrated as follow

$A(\omega, \theta)=\omega Q+P+2 \frac{K g \cos \theta}{\omega}$

Here $P$ and $K$ are constants of integration and $A$ is integral operator given by Eq. (66). Using homogeneity of function $F$ in Eq.(66)

$F\left(\varepsilon \omega, \varepsilon \omega_{1}, \varepsilon \omega_{2}, \varepsilon \omega_{3}\right)=\varepsilon^{12} F\left(\omega, \omega_{1}, \omega_{2}, \omega_{3}\right) \sim g^{-4} \omega^{12}(71)$ one can define the most general stationary Kolmogorov-type solution of the kinetic Eq. (60)

$N(\omega, \theta)=\frac{g^{4 / 3} P^{1 / 3}}{\omega^{5}} R\left(\frac{\omega Q}{P}, 2 \frac{g K}{\omega P}, \theta\right)$

with the energy spectrum

$E(\omega, \theta)=\frac{g^{4 / 3} P^{1 / 3}}{\omega^{4}} R\left(\frac{\omega Q}{P}, 2 \frac{g K}{\omega P}, \theta\right)$

Let us study the most important special cases. Homogeneous solutions with vanishing fluxes of wave action and momentum $(Q=0, K=0)$ give the well known direct cascade solution (Zakharov and Filonenko, 1966)

$E(\omega, \theta)=C_{p} \frac{g^{4 / 3} P^{1 / 3}}{\omega^{4}}$

Here $C_{p}=R(0,0,0)$ is the Kolmogorov constant (the first Kolmogorov constant). Scaling invariance of the problem allows for constructing other power-like solutions corresponding to constant fluxes of wave action and momentum. Suppose $N(\omega, \theta)$ is an isotropic power-like function of frequency

$N(\omega) \sim \omega^{-x}$

From Eq. (70) and homogeneity property (Eq. 71) one gets

$$
A(\omega)=f(x) \omega^{(15-3 x)}
$$

Here $f(x)$ is a function of one variable to be calculated numerically. In fact, $f(x)$ can be expressed through $\Lambda(s)$ (see and Geogjaev and Zakharov, $2005^{1}$ and Eq. 35). For the direct cascade $(P \neq 0, Q=0, K=0)$

$$
A(\omega) \sim \text { const; } \quad x=5 ; \quad C_{p}^{3}=f(5)
$$

${ }^{1}$ Geogjaev, V. V. and Zakharov, V. E.: Hasselmann equation revisited, in preparation, 2005. 
The inverse cascade solution $(Q \neq 0)$ with a constant wave action flux gives (Zakharov and Zaslavskii, 1982)

$A(\omega) \sim \omega ; \quad x=14 / 3 ; \quad C_{q}^{3}=f(14 / 3)$

and

$N(\omega, \theta)=C_{q} \frac{g^{4 / 3} Q^{1 / 3}}{\omega^{14 / 3}}$

$E(\omega, \theta)=C_{q} \frac{g^{4 / 3} Q^{1 / 3}}{\omega^{11 / 3}}$

Quite similarly for constant momentum flux $(K \neq 0)$ one gets

$$
\begin{aligned}
& A(\omega, \theta) \sim \omega^{-1} ; \quad x=16 / 3 ; \quad C_{m}^{3}=f(16 / 3) \\
& N(\omega, \theta)=C_{m} \frac{g^{5 / 3} K^{1 / 3}}{\omega^{16 / 3}} h(\theta) \\
& E(\omega, \theta)=C_{m} \frac{g^{5 / 3} K^{1 / 3}}{\omega^{13 / 3}} h(\theta)
\end{aligned}
$$

From the symmetry consideration one has

$h(\theta)=-h(\pi-\theta)$

i.e. the solution (Eq. 78) is not positive at all angles $\theta$. Thus, general solution (Eqs. 70 and 72) is unlikely to be realized in a whole range of $(\omega, \theta)$-plane. An anisotropic generalization of the solution can be found assuming $Q=0$ and momentum flux to be small (Katz and Kontorovich, 1974; Katz et al., 1976)

$E(\omega, \theta)=\frac{g^{4 / 3} P^{1 / 3}}{\omega^{4}}\left(C_{p}+C_{m} g K \cos \theta /(\omega P)+\ldots\right)$

$C_{m}$ is known as the second Kolmogorov constant. Eq. (79) predicts isotropy of the Kolmogorov spectrum at $\omega \rightarrow \infty$. In fact, the observed wind wave spectra are anisotropic for arbitrary large frequencies. A possible explanation is that in real situations the momentum flux $K$ is a growing function of frequency.

There is an additional restriction on power-like solutions. Convergence of integrals in expression (Eq. 66) for $A$ requires (Geogjaev and Zakharov, 2005) ${ }^{1}$

$5 / 2<x<19 / 4$

Thus, Eqs. (73) and (78) are just formal solutions that can be valid in a finite domain on $(\omega, \theta)$-plane. This is a case of the direct cascade solution (Eq. 73) that is an analogue of the classical Kolmogorov spectrum of turbulence in incompressible fluid. The energy source is assumed at some small but finite wave frequencies and the energy dissipates in high frequency domain.

In our further consideration the spatial presentation of Kolmogorov's solutions will be useful for the direct cascade of energy

$N(\boldsymbol{k})=C_{p} \frac{P^{1 / 3}}{2 g^{2 / 3}|\boldsymbol{k}|^{4}}, \quad E(\boldsymbol{k})=C_{p} \frac{P^{1 / 3}}{2 g^{1 / 6}|\boldsymbol{k}|^{7 / 2}}$

and for the inverse cascade of wave action

$$
N(\boldsymbol{k})=C_{q} \frac{Q^{1 / 3}}{2 g^{1 / 2}|\boldsymbol{k}|^{23 / 6}}, \quad E(\boldsymbol{k})=C_{q} \frac{Q^{1 / 3}}{2|\boldsymbol{k}|^{10 / 3}}
$$

4.3 Stationary solutions for the kinetic equation in presence of sources and sinks

In this section we discuss briefly stationary homogenous solutions of the Hasselmann equation in presence of forcing and dissipation, more details for the consideration can be found in (Zakharov, 2004). For simplicity we study the isotropic case only. In this case the Hasselmann equation is written in the form

$S_{n l}+\beta(\omega) N=0$

or in terms of auxiliary function $A$

$\frac{1}{2} \frac{d^{2} A}{d \omega^{2}}+\beta(\omega) N=0$

Integrating Eq. (83) over $\omega$ one gets evident formulas

$$
\begin{aligned}
\int_{-\infty}^{\infty} \beta(\omega) N(\omega) d \omega & =0 \\
\int_{-\infty}^{\infty} \omega \beta(\omega) N(\omega) d \omega & =0
\end{aligned}
$$

Conditions (Eq. 84) are satisfied only if $\beta(\omega)$ changes the sign at least twice. If $\beta(\omega)$ is negative for small and high frequencies, i.e. there are both low- and high-frequency dissipation, the energy spectrum $E(\omega)$, fluxes of wave action and energy take the forms presented in Fig. 6. The distributions for stationary solutions can be found from numerical integration of Eq. (82) or analysed qualitatively starting with a spectrum and using evident identities (Eq. 68). One gets

$$
\begin{aligned}
& Q(\omega)=-\int_{0}^{\omega} \beta(\tilde{\omega}) N(\tilde{\omega}) d \tilde{\omega} \\
& A(\omega)=\int_{0}^{\omega} Q(\tilde{\omega}) d \tilde{\omega} \\
& P(\omega)=A(\omega)-\omega Q(\omega)
\end{aligned}
$$

The qualitative analysis is illustrated by Fig. 6 basing on our numerical experiments where results by Komatsu and Masuda (1996) have been reproduced. The Hasselmann whitecapping dissipation arrested the spectrum downshift in these experiments for rather long time (approximately $100 \mathrm{~h}$ for the sketch discussed). This is the well-known effect of saturation (maturity) of wind-wave spectra studied numerically by Komen et al. (1984). Here we treat it as a balance of spectral fluxes due to nonlinear transfer and local (in wave scales) forcing. Both direct and inverse cascading coexist in the presented example.

The saturated spectrum has no pronounced peakedness in contrast to developing wave case. Nonlinear transfer term $S_{n l}$ is balanced by source function (Fig. 6b) but keeps "weakly turbulent" high-frequency tail $\omega^{-4}$ perfectly well (Fig. 6a). Limits of positive rates $\beta(\omega)$ are not easy to relate with generic properties of the stationary problem (Eq. 85) due to weak non-stationarity and anisotropy of the numerical example, this is why maxima of fluxes of wave action $Q$ (Fig. 6c) and energy $P$ (Fig. 6d) $P$ are slightly upper-shifted relatively 

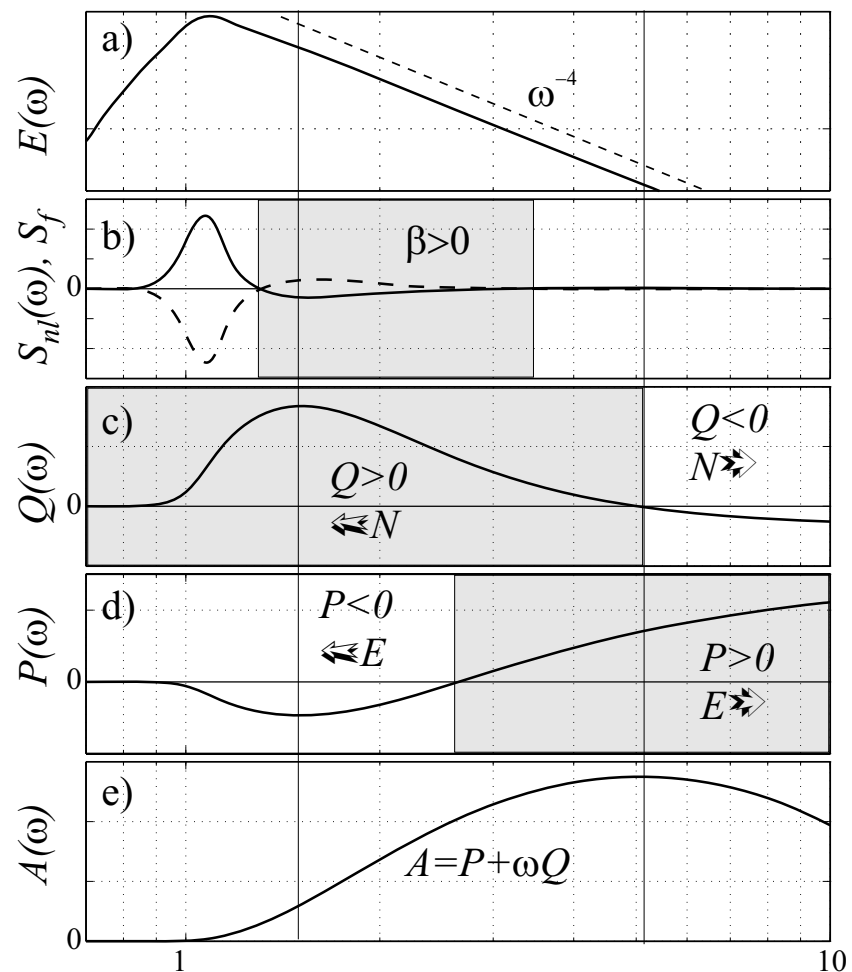

Fig. 6. (a) - Energy spectrum $E(\omega)$; (b) - nonlinear transfer $S_{n l}(\omega)$ and forcing term $S_{f}(\omega)$; (c) - wave action flux $Q ;(\mathbf{d})$ - energy flux $P$; (e) - auxiliary function $A(\omega)$ for quasi-stationary solution of the kinetic equation with Komatsu and Masuda (1996) setup: wave input (Eq. 47) by Snyder et al. (1981) at $U_{5}=20 \mathrm{~m} \cdot \mathrm{s}^{-1}$, Hasselmann's white-capping dissipation (Eq. 54), time $100 \mathrm{~h}$. Domains of positive $\beta, Q$ and $P$ are shaded. Vertical straight lines are given for maximal and zero wave action to show that the numerical solution obey identities for spectral fluxes (Eqs. 68 and 85).

to $\beta(\omega)$ zero-crossing. All other relations between fluxes and auxiliary function $A(\omega)$ are in excellent agreement with Eq. (85).

An additional example of "flux nature" of the Hasselmann equation solutions in presence of forcing is presented in Fig. 7. The structure of the solution is especially simple as far as domains of dissipation and input are well-separated. The dissipation takes place in the region of small frequencies $\omega<\omega_{0}$ and high frequencies $\omega>\omega_{2}$, the forcing is concentrated in a narrow range near $\omega \simeq \omega_{1}$, and $\omega_{0} \ll \omega_{1} \ll \omega_{2}$. Then in the range $\omega_{0}<\omega<\omega_{1}$ one gets a pure inverse cascade that describes the spectrum $\omega^{-11 / 3}$, while in the range $\omega_{1}>\omega>\omega_{2}$ - a pure direct cascade $N_{\omega} \simeq \omega^{-4}$. This situation is illustrated by Fig. 7 where the results of a numerical experiment are presented. Note that despite of strong anisotropy of input the numerical solution reflects the robust features of nonlinear transfer in wind-wave spectra fairly well. In more realistic situation (e.g. Fig. 6), when the wave forcing occupies a wide frequency domain the direct and the inverse cascades coexist and their discrimination does not manifest itself in such pronounced form.
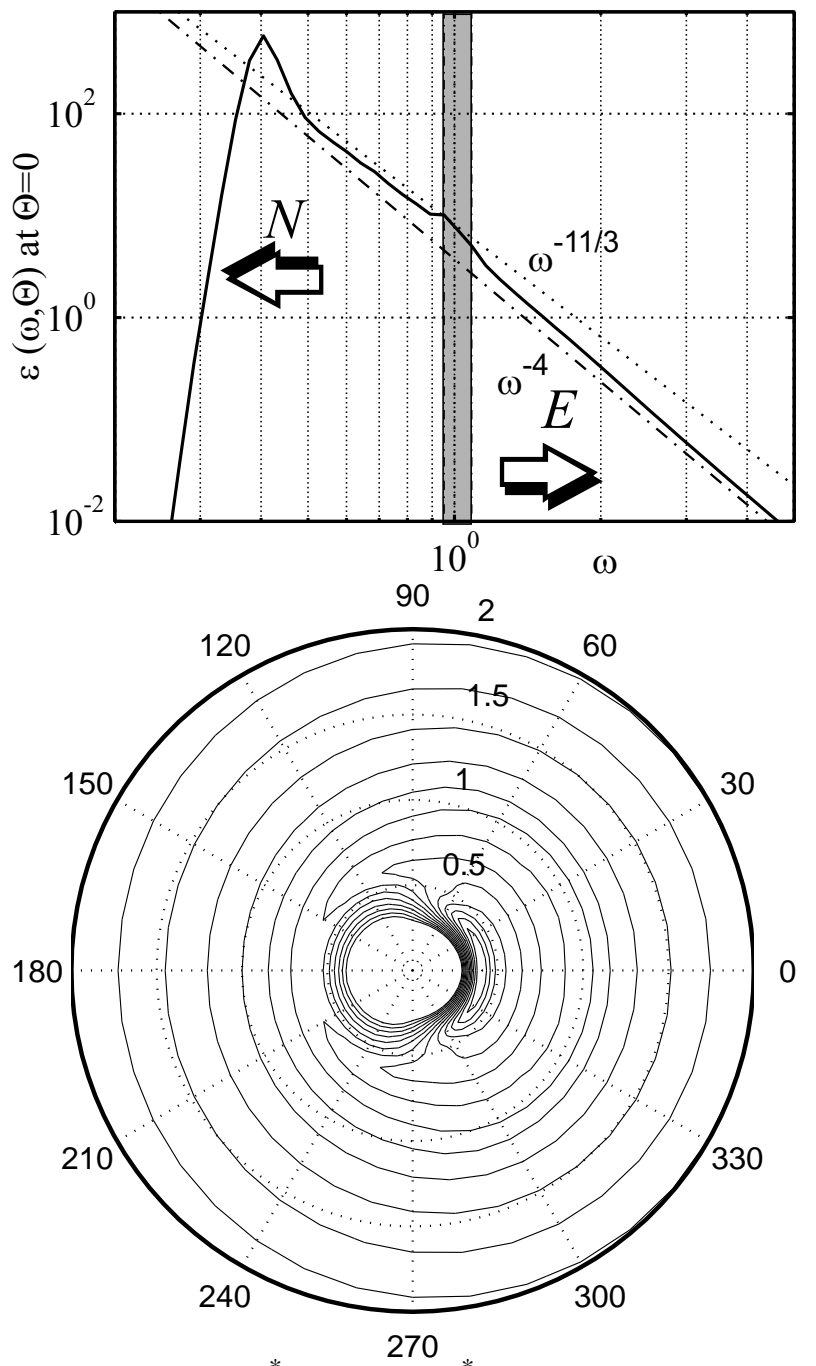

$\mathrm{T}=25.6 \mathrm{hrs} ; \mathrm{t}^{*}=122065 ; \omega^{*}=0.40$

Fig. 7. Form of the energy spectra $E(\omega, \theta)$ and its directional distribution (bottom) for an "artificial" source function. The solution is tending to Kolmogorov's asymptotics, i.e. to the inverse cascade in low frequencies and to the direct cascade in high frequencies.

\section{Self-similar solutions for kinetic equation}

In this section we present new theoretical results on windwave spectra evolution based on the theory of weak turbulence. These results can be considered as a generalization of the Kolmogorov-Zakharov constant flux solutions for the case of non-stationary (non-homogeneous) isotropic windwave spectra. The exponents of wind-wave spectra are usually discussed as a justification of physical relevance of the Kolmogorov spectra approach. At high frequency tails, the wind wave spectra keep exponent $(-4)$ that corresponds to the direct cascade solution (Eq. 73). At the same time, a part of wind wave community considers the $\mathrm{KZ}$ solutions as physically irrelevant because two key features of windwave spectra - the pronounced peakedness and the strong anisotropy - are, evidently, beyond the stationary isotropic 
power-like Kolmogorov-Zakharov solutions. In fact, the Kolmogorov-Zakharov exponents remain extremely robust for wind-wave spectra strongly localized in wave scales and directions. Figure 7 mentioned above illustrates an "unrealistic" case when relatively strong generation is localized in a narrow frequency range to leave more space for cascading regimes at lower and higher frequencies, i.e.

$\beta(\omega, \theta)=\left\{\begin{array}{cc}\text { Const, } \omega_{1}<\omega<\omega_{2} ; \quad \theta_{1}<\theta<\theta_{2} \\ 0, & \text { otherwise }\end{array}\right.$

This forcing, as well as the resulting spectrum, is strongly anisotropic. In spite of this fact, this spectrum keeps the Kolmogorov-Zakharov exponents in a rather wide range of angles and frequencies.

In low frequencies (relatively to generation domain) the solution is approximated by power-like dependence $\omega^{-11 / 3}$ corresponding to the inverse cascade regime. For high frequencies the solution tends to the law $\omega^{-4}$, i.e. to the direct cascade asymptotics. Thus, both features of real wind-wave spectra and of basic weak turbulence models are coexisting within this numerical solution.

The key point of our theoretical analysis is scaling invariance (Eq. 28) of the conservative kinetic Eq. (4) for the case of deep water waves. This invariance allows to construct a family of anisotropic self-similar solutions for nonstationary and non-homogeneous kinetic equation. The stationary Kolmogorov-Zakharov solutions can be considered as special solutions of the family at $t \rightarrow \infty$. Thus, the KZ solutions can be treated not only as mathematical objects but as physically relevant states of the wave field at infinitely long time $t \rightarrow \infty$.

5.1 The kinetic equation for deep water waves - homogeneity of collision integral and routes to self-similarity

For deep water case there is no specific scaling and nondimensional variables can be introduced in arbitrary way

$$
\begin{aligned}
t & =\tau / \omega_{0}, \quad \boldsymbol{x}=\chi / k_{0}, \quad \boldsymbol{k}=k_{0} \boldsymbol{\kappa} ; \\
\omega & =\omega_{0} \Omega, \quad \Omega=\sqrt{\boldsymbol{\kappa}}, \quad \omega_{0}=\sqrt{g k_{0}}
\end{aligned}
$$

Non-dimensional wave action takes a form

$$
N(\boldsymbol{k})=\frac{1}{\omega_{0} k_{0}^{4}} \widetilde{n}(\boldsymbol{\kappa})=\frac{g^{4}}{\omega_{0}^{9}} \widetilde{n}(\boldsymbol{\kappa})
$$

Correspondingly

$N(\omega, \theta)=\frac{g^{2}}{\omega_{0}^{6}} \widetilde{n}(\omega) ; \quad E(\omega, \theta)=\frac{g^{2}}{\omega_{0}^{5}} \widetilde{\varepsilon}(\omega, \theta)$

Below we omit tilde in non-dimensional wave action notations. We shall relate characteristic scales $k_{0}, \omega_{0}$ to the only "external" parameter of wave generation - wind speed $U_{10}$ at standard height $\left(u_{*}-\right.$ friction velocity can be used as well). Thus, unless otherwise is specified, we define characteristic wavenumber and wave frequency scales as follows

$k_{0}=g / U_{10}^{2}, \quad \omega_{0}=g / U_{10}$
The non-dimensional energy and frequency become

$$
\begin{aligned}
& \epsilon=\int \sqrt{\kappa} n(\boldsymbol{\kappa}) d \boldsymbol{\kappa}=k_{0}^{2} \sigma^{2}=g^{2} \sigma^{2} / U_{10}^{2} \\
& v=\frac{\int \kappa n(\boldsymbol{\kappa}) d \boldsymbol{\kappa}}{\int \sqrt{\kappa} n(\boldsymbol{\kappa}) d \boldsymbol{\kappa}}=\bar{\omega} / \omega_{0}
\end{aligned}
$$

The non-dimensional cyclic frequency of spectral peak is the well known inverse wave age

$\omega_{*}=\omega_{p} / \omega_{0}=\omega_{p} U_{10} / g$

The kinetic equation for water waves in homogeneous ocean (Eq. 2) can be rewritten in non-dimensional form as follows

$$
\frac{\partial n}{\partial \tau}+\frac{\kappa \nabla n}{2|\kappa|^{3 / 2}}=\widetilde{S}_{n l}+\widetilde{S}_{i n}+\widetilde{S}_{d i s s}
$$

It is of primary importance for further analysis that collision integral $S_{n l}$ is homogeneous in wavenumber (see Eq. 29) as well as the terms in left-hand side of Eq. (87). It gives an idea to construct self-similar solutions for stationary (fetchlimited) and homogeneous (duration-limited) cases. Unfortunately, terms $S_{i n}$ and $S_{d i s s}$ are not homogeneous functions of $\kappa$. Thus, the analysis of self-similar solutions of the conservative kinetic Eq. (4) can give physically relevant results if external forcing terms are small. Look for such solutions assuming formally the smallness of generation and dissipation terms.

\subsection{Self-similar solutions for duration-limited wave growth}

Consider the waves generated in homogeneous sea by stationary wind from an initial state. The so-called duration limited case gives the following kinetic equation

$$
\frac{\partial n}{\partial \tau}=\widetilde{S}_{n l}+\widetilde{S}_{f}
$$

Searching for the solutions in the form

$n(\boldsymbol{\xi}, \tau)=a \tau^{\alpha} U_{\beta}(\boldsymbol{\xi}, \tau)$

where $\xi=b \kappa \tau^{\beta}$, one gets after simple algebra

$$
\begin{gathered}
\tau \frac{\partial U}{\partial \tau}+\beta \xi \nabla_{\xi} U_{\beta}(\xi, \tau)+\alpha U_{\beta}(\xi, \tau)= \\
\frac{a^{2}}{b^{19 / 2}} \tau^{2 \alpha+1-19 \beta / 2} \widetilde{S}_{n l}\left[U_{\beta}(\xi, \tau)\right]+\frac{S_{f}(\xi, \tau)}{a \tau^{\alpha-1}}
\end{gathered}
$$

Condition

$\alpha=\frac{19 \beta-2}{4}$

leaves two terms only in Eq. (89) that contain dependence on time explicitly: the term with time derivative and the term of external forcing $S_{f}$. For $\alpha>1$ the latter term vanishes asymptotically with time (we detail this point below), thus, one can look for approximate stationary solutions of the kinetic Eq. (89) in variables $(\xi, \tau)$. After re-scaling

$a=b^{19 / 4}$ 
the stationary "shape" function $U_{\beta}(\xi)$ obeys the following integro-differential equation

$$
\begin{aligned}
& {\left[\alpha U_{\beta}+\beta \xi \nabla_{\xi} U_{\beta}\right]=\pi \int d \xi_{1} d \xi_{2} d \xi_{3}\left|T_{\xi_{1}} \xi_{2} \xi_{3}\right|^{2} } \\
\times & \delta\left(\xi+\xi_{1}-\xi_{2}-\xi_{3}\right) \\
\times & \delta\left(\sqrt{|\xi|}+\sqrt{\left|\xi_{1}\right|}-\sqrt{\left|\xi_{2}\right|}-\sqrt{\left|\xi_{3}\right|}\right) \\
\times & {\left[U_{\beta}\left(\xi_{1}\right) U_{\beta}\left(\xi_{2}\right) U_{\beta}\left(\xi_{3}\right)+U_{\beta}\left(\xi_{)} U_{\beta}\left(\xi_{2}\right) U_{\beta}\left(\xi_{3}\right)\right.\right.} \\
& \left.-U_{\beta}(\xi) U_{\beta}\left(\xi_{1}\right) U_{\beta}\left(\xi_{2}\right)-U_{\beta}(\xi) U_{\beta}\left(\xi_{1}\right) U_{\beta}\left(\xi_{3}\right)\right]
\end{aligned}
$$

Total amount of solutions for Eq. (91) is not known. Formally we have a two-parametric family of self-similar solutions with independent parameters $\alpha$ and $a$. The parameter $a$ can be related to initial conditions while parameter $\alpha$ is specified by conditions of consistency with higher approximations in a formally small parameter of wave pumping $\varrho$ (see Eq. 45).

Let the total wave action $N$ is a power-like function of time

$N_{t o t} \sim \int n(\boldsymbol{\xi}, \tau) d \boldsymbol{k} \sim b^{11 / 4} \tau^{r_{\tau}}$

Using the self-similar form of $n(\xi, \tau)$ one gets for the total wave action growth rate

$$
r_{\tau}=\alpha-2 \beta=(11 \beta-2) / 4=(11 \alpha-4) / 19
$$

Solutions of this type make sense if "perturbation" term dealing with the effect of generation/dissipation in Eq. (89) vanishes with time, i.e.

$\alpha>1 ; \quad r_{\tau}>7 / 19$

and solutions do not grow infinitely in the frequency range of wave generation. More accurate estimates of the effect of forcing, and, hence, of validity of our approximation can be carried out for particular cases of wave growth.

\subsubsection{Swell - "purely nonlinear" evolution of wave field}

The special case of wave swell when generation and dissipation are absent gives (see Eq. 92)

$r_{\tau}=0 ; \quad \beta=2 / 11 ; \quad \alpha=4 / 11$

The solution

$n=a \tau^{4 / 11} U_{2 / 11}\left(b \kappa \tau^{2 / 11}\right)$

describes downshift of spectral peak as

$\left|\boldsymbol{k}_{p}\right| \sim \tau^{-2 / 11}$

The total energy decreases as

$E_{t o t} \sim \tau^{-1 / 11}$

Note, that the total wave action is real constant of motion in this case while the wave energy leaks to small scales as it was described in previous section.

We cannot guarantee that the corresponding self-similar solutions appear as a result of evolution from an arbitrary
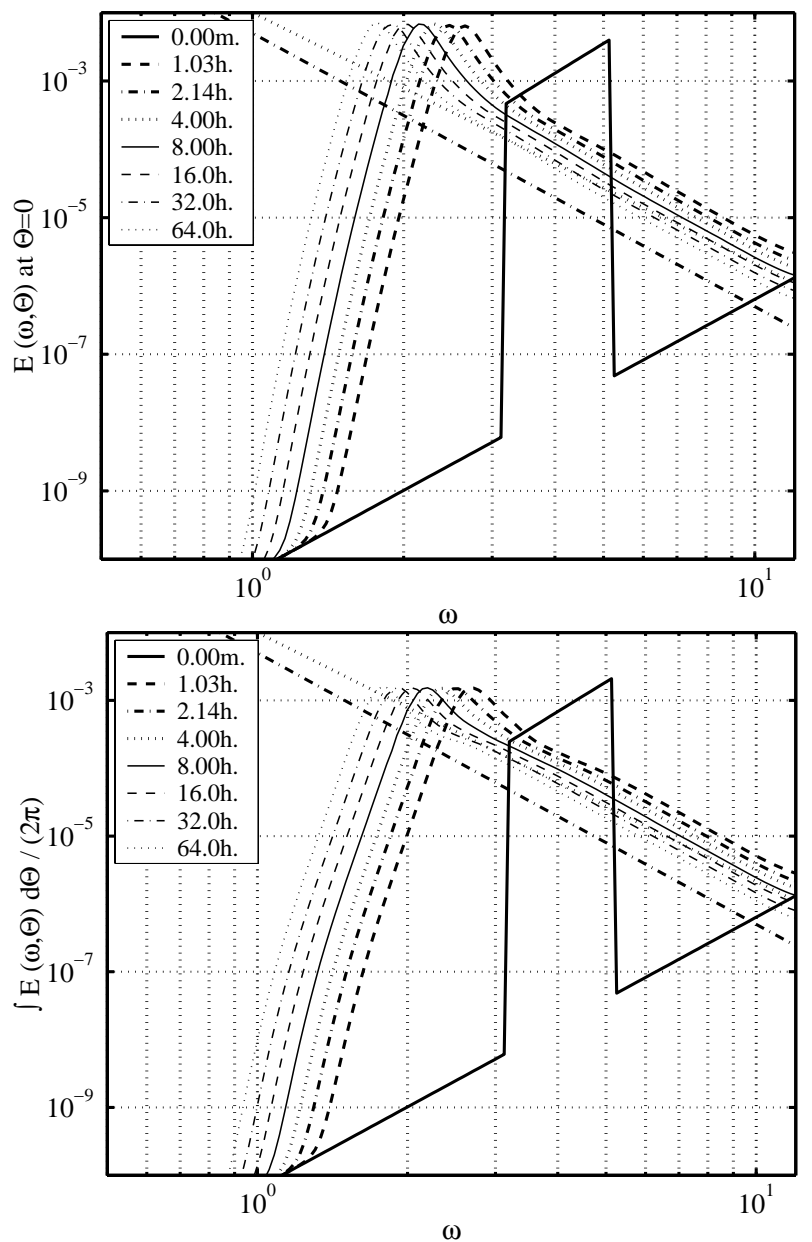

Fig. 8. Numerical solutions for the swell case. Both directional (top panel) and one-dimensional (bottom - averaged in direction) spectra demonstrate features of self-similarity. Time in hours is shown in legends.

initial conditions. Fortunately, this is not our case. Below we present numerous justifications of self-similarity features for variety of initial data and for different conditions of wave generation and dissipation. Here we give just the first illustration as a motivation for further study.

Figure 8 shows the evolution of step-like initial spectrum with no dissipation or generation. The initial state corresponds to rather steep waves $(a k=0.225)$ and mean wavelength $5 \mathrm{~m}$. After $64 \mathrm{~h}$ the dominant waves become approximately 3 times longer and their steepness 5 times less. The numerical solution for the kinetic equation shows selfsimilarity features perfectly well (Fig. 8). The peak frequency $\omega_{p}$ and "shape function" $U_{2 / 11}$ can be extracted easily to justify these features (Fig. 9). Top Fig. 9 shows that the solution peak is travelling very close to the theoretical self-similar dependence

$\left(\xi_{p}\right)^{1 / 2} \sim \omega_{p} t_{p}^{1 / 11} \sim\left|\boldsymbol{k}_{p}\right|^{1 / 2} t_{p}^{1 / 11}=\mathrm{const}$ 

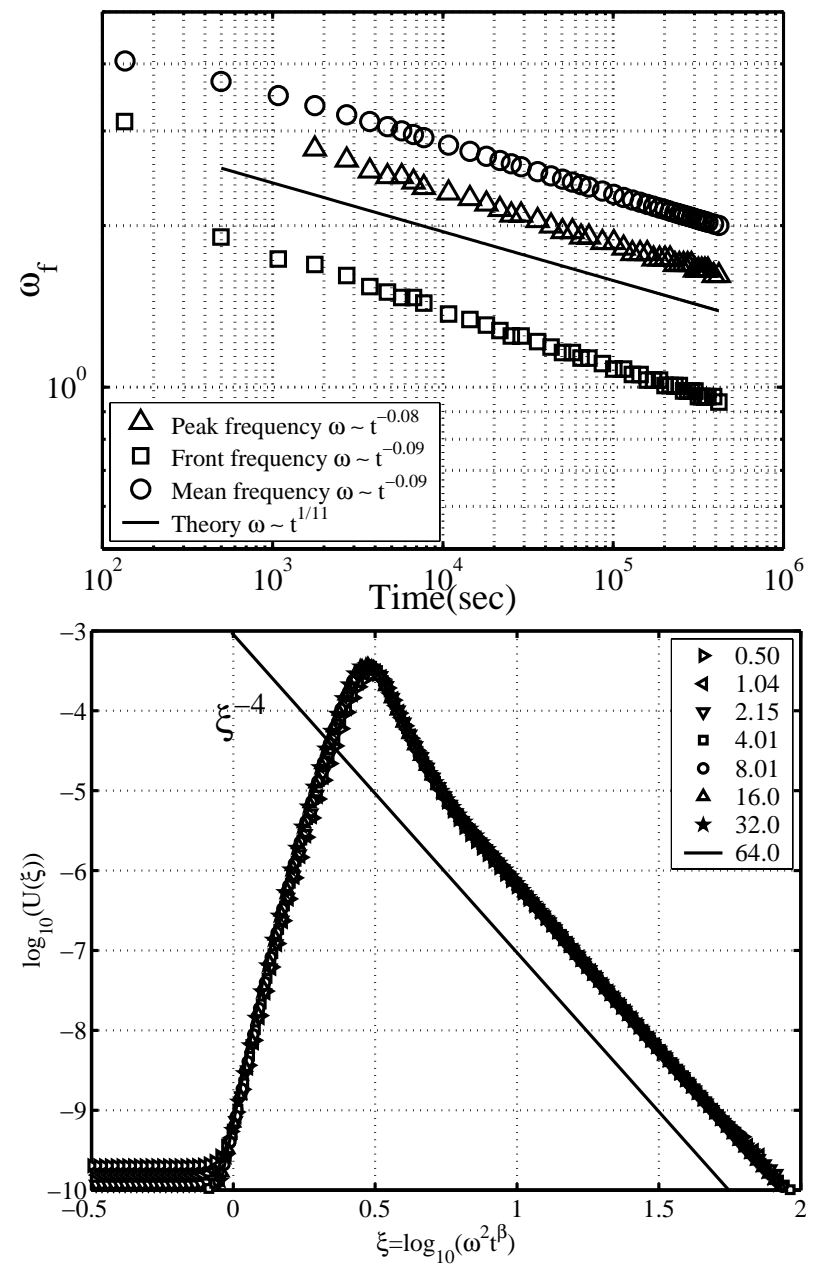

Fig. 9. Self-similarity of the kinetic equation solutions for the case of swell. Top - dependence of mean (circles), peak (triangles) and front (squares - maximal positive spectral slope) frequencies on time. The exponents of downshift $\beta$ are close to the theoretical value $\beta / 2=1 / 11$. Bottom - function $U_{2 / 11}(\xi)\left(\xi=\omega^{2} t^{2 / 11}\right)$ extracted from the numerical solutions at different times (see legend, in hours). The direct cascade slope $\xi^{-4}$ is shown by hard line

The "shape function" $U_{2 / 11}(\xi)$ extracted for different times (Fig. 9, bottom) shows remarkably strong tendency to selfsimilar behavior: after half an hour no difference is seen for the functions. All the evolution occurs for the low-frequency pedestal only. This is illustration of a trivial fact: the nonlinearity is stronger - the evolution to self-similar form is faster.

As we noted above, the total wave action is conserved for the swell case while the energy leaks. This leakage provides a direct cascade of energy to high-frequency range described by the Zakharov and Filonenko (1966) spectrum $E(\omega) \sim \omega^{-4}$ as it is clearly displayed by Fig. 9 .

\subsubsection{Self-similar solutions for time-dependent wave input}

The self-similar solution for the swell (Sect. 5.2.1) is an exact solution of the kinetic equation. For wind-driven waves the self-similar solutions are nothing but approximate ones. In fact, we have a large family of the approximate solutions corresponding to different exponents of wave action growth rate $r_{\tau}$. This family gives a basis for comparison and interpretation of numerical results of next sections.

Defining integrals

$A_{\beta}=\int \sqrt{|\xi|} U_{\beta}(\xi) d \xi \quad B_{\beta}=\int|\xi| U_{\beta}(\xi) d \xi$

one can express mean frequency and total energy for selfsimilar solutions (Eq. 88) as follows

$$
\begin{aligned}
\epsilon=u_{\tau} \tau^{p_{\tau}} & v=v_{\tau} \tau^{-q_{\tau}} \\
u_{\tau}=b^{9 / 4} A_{\beta} ; & v_{\tau}=b^{-1 / 2} B_{\beta} / A_{\beta}
\end{aligned}
$$

with exponents

$p_{\tau}=(9 \beta-2) / 4 ; \quad q_{\tau}=\beta / 2$

For the wave action growth rate $r_{\tau}$ one has

$r_{\tau}=\alpha-2 \beta=(11 \beta /-2) / 4$

Total wave action grows as

$n_{t o t}=\frac{\epsilon}{v}=b^{11 / 4} \tau^{r} \int U_{\beta}(\xi) d \xi$

and for total momentum one has

$M_{t o t}=\int|\boldsymbol{\xi}| n(\boldsymbol{\xi}, \tau) \cos \theta d \boldsymbol{k} \sim M_{\theta} b^{7 / 2} \tau^{m_{\tau}}$

where

$m_{\tau}=\alpha-3 \beta=(7 \beta-2) / 4$

Additional dimensionless parameter $M_{\theta}$ is introduced in Eq. (95) to emphasize dependence of total momentum on angular spreading of self-similar solutions. For isotropic spectra $M_{\theta}=0$. There might exist some "most anisotropic" self-similar spectrum with maximal $M_{\theta}$. We can guess that exactly this spectrum that provides the maximal momentum transport is realized in the real sea.

Dependence of mean energy on mean frequency gives

$\epsilon=u_{\tau} v_{\tau}^{p_{\tau} / q_{\tau}} v^{-2 T}$

Parameters

$B_{T o b a}=b^{9 / 4-T} B_{\beta}^{2 T} ; \quad T=\frac{9 / 2-1 / \beta}{2}$

can be related to the well-known Toba's law (see Eqs. 43, 94 and Toba, 1973). Note, that for the definition (Eq. 97) the Toba exponent depends on wave growth rate $p_{\tau}$ (or $r_{\tau}$ ). Two particular cases are of special interest. 
5.2.3 Constant wave action input and the KolmogorovZakharov inverse cascade solution

For the case of linear growth of total wave action one has the following set of exponents

$$
\begin{aligned}
& \alpha=23 / 11 ; \quad \beta=6 / 11 ; \\
& r_{\tau}=(11 \beta-2) / 4=1 ; \quad p_{\tau}=8 / 11 \approx 0.73 ; \\
& q_{\tau}=3 / 11 \approx 0.27 ; \quad T=\frac{9 / 2-1 / \beta}{2}=4 / 3
\end{aligned}
$$

i.e. the amplitude growth is slower than Toba's law predicts (Toba, 1973). Assuming that the self-similar solution

$n=a \tau^{23 / 11} U_{6 / 11}\left(b \kappa \tau^{6 / 11}\right)$

has power-like high-frequency asymptotics one can find the exponent corresponding exactly to the inverse cascade solution (Eq. 81). Thus, the homogeneous stationary Kolmogorov-Zakharov solution (Eqs. 76 and 81) can be related quite naturally with this non-stationary self-similar regime. This link becomes more definite if we substitute the inverse cascade solution into Eq. (91). Left- and right-hand sides of the equation vanish simultaneously. We come to remarkable physical (and mathematically trivial) result: The Kolmogorov-Zakharov inverse cascade solution is the exact solution for the homogeneous kinetic equation in self-similar variables (Eq. 91)! For the inverse cascade KZ solution (Eqs. 76 and 81) the balance of non-stationarity (left-hand side of Eq. 91) and nonlinearity (right-hand side of Eq. 91) is split for the "shape function" $U_{\beta}(\xi)$.

5.2.4 Constant wave energy input and the KolmogorovZakharov direct cascade solution

Quite similar to the previous paragraph one can obtain for the regime of constant wave energy input $r_{\tau}=4 / 3\left(p_{\tau}=1\right)$

$$
\begin{array}{cl}
\alpha=8 / 3 ; & \beta=2 / 3 ; \\
r_{\tau}=(11 \beta-2) / 4=4 / 3 ; & p_{\tau}=1 ; \\
q_{\tau}=1 / 3 ; & T=\frac{9 / 2-1 / \beta}{2}=3 / 2
\end{array}
$$

The Toba exponent $3 / 2$ appears to be consistent with the set of exponents and corresponds to the wave action input growing with time while energy input remains constant.

Once again, assuming power-like high-frequency tails for the non-stationary self-similar solution one gets that the Kolmogorov-Zakharov direct cascade solution (Eq. 80) is its stationary counterpart. Quite similar to the previous section the Kolmogorov-Zakharov direct cascade appears to be the exact solution for the homogeneous kinetic equation in selfsimilar variables (Eq. 91)!

The special cases of duration-limited wind wave growth are summarized in Table 2 .

It should be emphasized, that the self-similar solutions (88) are not necessary isotropic. The contributions of nonlinear transfer and wave input can differ dramatically for different directions and, hence, self-similar behaviour can be reached in different ways for different directions or cannot be reached at all. The result depends on interplaying of nonlinear and input terms, that forms some kind of "magic circle": low input means low nonlinear transfer and, thus, the regime of dominating nonlinear transfer may be unattainable for reasonable time: this is a subject of numerical analysis. 5.3 Self-similar solutions and flux balance in the kinetic
equation

Equation (91) for $U_{\beta}(\xi)$ has a form

$S_{n l}\left(U_{\beta}(\xi)\right)=\beta \xi \nabla_{\xi} U_{\beta}(\xi)+\alpha U_{\beta}(\xi)$

(see Eq. 89 at $t \rightarrow \infty$ ) and can be considered as a basic one in the weak turbulence theory. It can be treated quite naturally as a stationary kinetic equation with specific external forcing quite similarly to the stationary equation in primitive variables (Eq. 82). In contrast to Eq. (82) the external forcing is not small yet because the right-hand term describes strong effect of non-stationarity. Equation (98) gives an accurate account of interplaying of spectral distributions and spectral fluxes for wind wave field. Note, that the coefficients $\alpha$ and $\beta$ are constants and the right-hand-side of Eq. (98) is linear in function $U_{\beta}$. Thus, the qualitative analysis of Sect. 4.3 can be amplified by explicit and mathematically correct results for the family of approximate self-similar solutions.

The first important result is a behaviour of the collision integral $S_{n l}$ at large time. If our hypothesis on dominating nonlinear transfer is correct and the solutions do tend to the asymptotic self-similar forms that satisfy (Eq. 98), the $S_{n l}$ is simply a linear combination of the spectral form and its first derivative. Assume the JONSWAP spectrum to be close to an asymptotic self-similar solution. As soon as the right-hand side of Eq. (98) is linear in $U_{\beta}$ one can take (see Eq. 37)

$U_{\beta}(\xi)=\xi^{-4} \exp \left(-\xi^{-2}\right) \exp \left\{\ln \gamma \cdot \exp \left(-\frac{(\sqrt{\xi}-1)^{2}}{2 \sigma_{p}^{2}}\right)\right\}$

and to reconstruct the corresponding asymptotic behavior of collision integral $S_{n l}$ and spectral fluxes. The results are shown in Fig. 10. Parameter $\sigma_{p}=0.08$ and three different peakedness $\gamma=1,3.3,5$ have been taken for calculations. Left column of Fig. 10 shows results for the inverse cascade set of exponents $r_{\tau}=1, p_{\tau}=8 / 11, \alpha=23 / 11, \beta=6 / 11$ and the swell case $\left(r_{\tau}=0, p_{\tau}=-1 / 11, \alpha=4 / 11, \beta=2 / 11\right)$ is illustrated by the right column of plots. One can see a strong effect of peakedness on all the patterns presented in Fig. 10. The case $\gamma=1$ (an analogue of the Pierson-Moskowitz spectrum) does not give a typical strongly localized pattern for $S_{n l}$ (Fig. 10c) in contrast to other cases. Stress the resemblance of the patterns and the exact collision integral for JONSWAP spectra in Fig. 3 and in previous papers (Hasselmann et al., 1985; Masuda, 1986; Komatsu and Masuda, 1996). The similarity is more pronounced for sharp spectra $(\gamma=3.3,5)$ than for $\gamma=1$ (the Pierson-Moskowitz spectrum). The latter implies that JONSWAP spectra are rather close to inherent wind-wave spectral shapes. 
Table 2. Exponents of self-similar solutions in duration-limited case.

\begin{tabular}{ccccccl}
\hline$r_{\tau}$ & $\alpha=\frac{19 r+4}{11}$ & $\beta=\frac{4 r+2}{11}$ & $p_{\tau}=\frac{9 r-1}{11}$ & $q_{\tau}=\frac{2 r+1}{11}$ & $T$ & Regime \\
\hline 0 & $4 / 11$ & $2 / 11$ & $-1 / 11$ & $1 / 11$ & $-1 / 2$ & Swell \\
1 & $23 / 11$ & $6 / 11$ & $8 / 11$ & $3 / 11$ & $4 / 3$ & Constant wave action input \\
$4 / 3$ & $8 / 3$ & $2 / 3$ & 1 & $1 / 3$ & $3 / 2$ & Constant wave energy input \\
\hline
\end{tabular}

Table 3. Exponents of fluxes evolution for self-similar solutions in duration-limited case.

\begin{tabular}{ccccl}
\hline$r_{\tau}$ & $S_{n l}(\boldsymbol{k}, t) \sim t^{s} S_{n l}(\boldsymbol{\xi})$ & $Q(\boldsymbol{k}, t) \sim t^{s_{q}} Q(\boldsymbol{\xi})$ & $P(\boldsymbol{k}, t) \sim t^{s_{p}} P(\boldsymbol{\xi})$ & Regime \\
\hline 0 & $s=(19 r-7) / 11$ & $s_{q}=(r-1)$ & $s_{p}=(9 r-12) / 11$ & \\
1 & $-7 / 11$ & -1 & $-12 / 11$ & Swell \\
$4 / 3$ & $12 / 11$ & 0 & $-3 / 11$ & Constant wave action input \\
\hline
\end{tabular}

Spectral fluxes for the stationary Eq. (98) can be calculated explicitly as integrals of its right-hand side (see Eqs. 62-64).

$$
\begin{aligned}
& Q=\int_{-\pi}^{\pi} \int_{0}^{|\boldsymbol{\xi}|}\left(\beta|\boldsymbol{\xi}|^{2} \frac{\partial U_{\beta}}{\partial|\boldsymbol{\xi}|}+\alpha|\boldsymbol{\xi}| U_{\beta}\right) d|\boldsymbol{\xi}| d \theta \\
& =\left.\int_{-\pi}^{\pi} \beta|\xi|^{2} U_{\beta} d \theta\right|_{0} ^{|\xi|}+r_{\tau} \int_{-\pi}^{\pi} \int_{0}^{|\xi|}|\xi| U_{\beta} d|\xi| d \theta \\
& P=-\int_{-\pi}^{\pi} \int_{0}^{|\xi|}\left(\beta|\xi|^{5 / 2} \frac{\partial U_{\beta}}{\partial|\boldsymbol{\xi}|}+\alpha|\xi|^{3 / 2} U_{\beta}\right) d|\boldsymbol{\xi}| d \theta= \\
& -\left.\int_{-\pi}^{\pi} \beta|\xi|^{5 / 2} U_{\beta}(\xi) d \theta\right|_{0} ^{|\xi|} \\
& -p_{\tau} \int_{-\pi}^{\pi} \int_{0}^{|\xi|}|\xi|^{3 / 2} U_{\beta} d|\xi| d \theta \\
& K=-\int_{-\pi}^{\pi} \int_{0}^{|\xi|}\left(\beta|\xi|^{3} \frac{\partial U_{\beta}}{\partial|\xi|}+\alpha|\xi|^{2} U_{\beta} d|\xi|\right) d \theta= \\
& -\left.\int_{-\pi}^{\pi} \beta|\xi|^{3} U_{\beta}(\xi) d \theta\right|_{0} ^{|\xi|}-m_{\tau} \int_{-\pi}^{\pi} \int_{0}^{|\xi|}|\xi|^{2} U_{\beta} d|\xi| d \theta
\end{aligned}
$$

The result of the integration is of fundamental interest: for positive exponents of wave action, energy and momentum growth $r_{\tau}, p_{\tau}, m_{\tau}$ the signs of fluxes $Q, P$ and $K$ are fixed and correspond to inverse cascade regime, i.e. $Q>0, P<0$, $K<0$. Note, that small rates $r_{\tau}<7 / 19$ are of little interest for our analysis because the smallness of source terms is questionable in this case (see Eqs. 93, 89).

The case of swell is of special interest. Parameters $p_{\tau}$, $m_{\tau}$ are negative and both types of cascades are co-existing for wave energy and momentum: inverse cascade in low frequency band (small $|\boldsymbol{\xi}|$ ) and leakage of energy and momentum (direct cascade) in high frequencies.

We assumed no angular dependence of $U_{\beta}(\xi)$ for Fig. 10 because of no qualitative differences for anisotropic distributions. Note a remarkable coincidence of our calculations with results of qualitative analysis of saturated wave spectrum in Sect. 4.3: the effect of non-stationarity can be treated as a source term in the framework of spectral peak (in other words, in self-similar variables). The "external forcing" in Eq. (98)

$S_{i n}^{*}=\beta \xi \nabla_{\xi} U_{\beta}+\alpha U_{\beta}$

arrests wave action and energy fluxes in low frequency band as it was described in Sect. 4.3 (see Fig. 6). Permanent forms of the self-similar solutions are reached due to this arrest. In high-frequency range the arresting effect of term $S_{i n}^{*}$ vanishes for power-like tails of the Kolmogorov-Zakharov solutions (see notes in Sects. 5.2.3 and 5.2.4 given by italic). Thus, fluxes in high-frequencies are not vanishing for the patterns of $U_{\beta}$ that implies a production of wave action and energy in the range. The physically important arrest of fluxes in small scales cannot be described within the asymptotic theory (the only exception is the swell case).

In Fig. 10d, e fluxes are tending to constant values quite rapidly. Note, that self-similar argument $\xi$ in the figure corresponds exactly to non-dimensional wavenumber $|\boldsymbol{k}| /\left|\boldsymbol{k}_{p}\right|=\omega^{2} / \omega_{p}^{2}$ and for $\xi=2$ the fluxes reach their $95 \%$ level of asymptotic values for the case of wave pumping (left column). The same tendency to the basic KolmogorovZakharov solutions (Eq. 70) is seen for the auxiliary function $A(\xi)(A(\omega))$.

Evidently, the full dependencies on time and primitive wavenumber $\boldsymbol{k}$ can be obtained easily for collision integral and fluxes. The corresponding transformation is a powerlike stretching of Fig. 10 in abscise and ordinate with time . The exponents of the transformation are given in Table 3.

Note, that fluxes depend on time. The special case $r_{\tau}=1$ (Table 3) corresponds to constant flux of wave action and can be associated with the inverse cascade KZ solution. The inverse cascade of energy decays as $\tau^{-3 / 11}$ in this case. The constant (in time) flux of energy gives the wave action flux growing with time as $\tau^{1 / 3}$. 

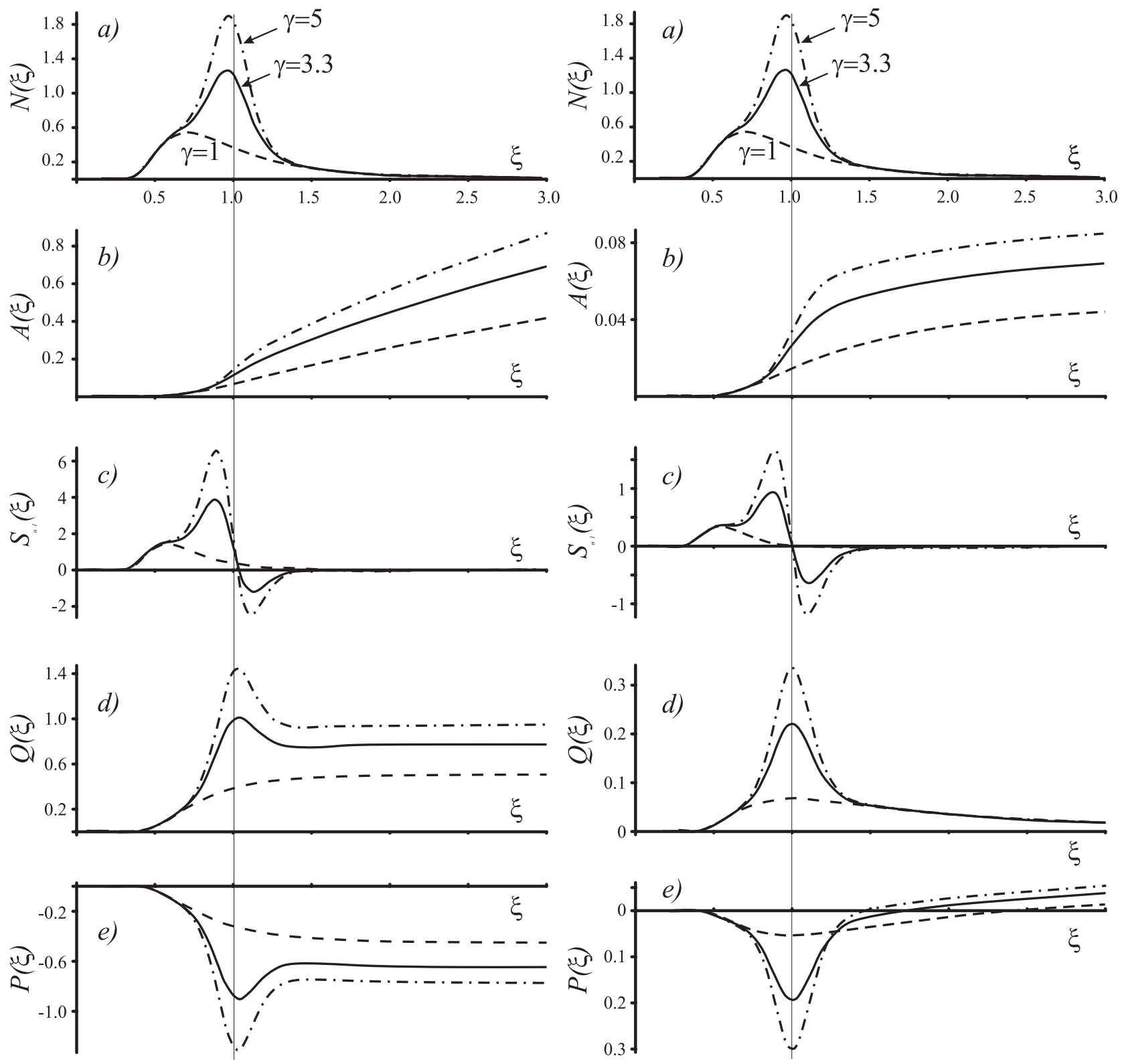

Fig. 10. Patterns of wave spectra, auxiliary function $A(\xi)$, collision integral $S_{n l}(\xi)$ and fluxes of wave action $Q(\xi)$ and energy $P(\xi)$ for the balance Eq. (98) for shape function $U_{\beta}$. The JONSWAP-like function of non-dimensional argument $\xi=|\boldsymbol{k}| /\left|\boldsymbol{k}_{p}\right|$ is taken as a shape function $U_{\beta}$. Curves for different peakedness parameter $\gamma=1$ (dashed), $\gamma=3.3$ (hard) and $\gamma=5$ (dash-dotted line) are given. Left column - linear wave action growth $\left(r_{\tau}=1, \alpha=23 / 11, \beta=6 / 11, p_{\tau}=8 / 11\right)$, right column - swell $\left(r_{\tau}=0, \alpha=4 / 11, \beta=2 / 11 p_{\tau}=-1 / 11\right)$.

\subsection{Self-similar solutions for fetch-limited case}

Extend the previous section analysis and recent results by Zakharov (2002) for the case of fetch-limited wave growth.

Consider an idealized problem of fetch-limited growth when constant offshore wind is perpendicular to the straight coast line and wind wave spectra depend on the only spatial coordinate $\chi$. The first-order "conservative" approximation $(\varrho=0)$ gives the balance

$\frac{\cos \theta}{2 \kappa^{1 / 2}} \frac{\partial n}{\partial \chi}=\widetilde{S}_{n l}$
Here $\kappa=|\kappa|$ - wavenumber, $\theta$ - polar angle of the wavevector relatively to wind direction, $\chi-x$-coordinate - fetch. The family of self-similar solutions for Eq. (99) can be written as:

$n(\kappa, \chi, \theta)=a \chi^{\alpha} P_{\beta}\left(b \chi^{\beta} \kappa, \theta\right)$

Substituting Eq. (100) into Eq. (99) one finds

$\alpha=5 \beta-1 / 2, \quad a=b^{5}$

Quite similarly to the previous section the "shape function" $P_{\beta}$ depends on two variables $-\theta$ and $\zeta=b \chi^{\beta} \kappa$. Function 
$P_{\beta}(\zeta)$ is independent of parameters $a$ and $b$ (see Eq. 101) and satisfies the following integro-differential equation:

$$
\begin{aligned}
& \frac{\cos \theta}{2 \sqrt{\zeta}}\left[\alpha P_{\beta}+\beta \zeta \frac{\partial P_{\beta}}{\partial \zeta}\right]=\pi \int \mid T_{\zeta \zeta_{1} \zeta_{2} \zeta_{3},\left.\theta \theta_{1} \theta_{2} \theta_{3}\right|^{2}} \\
& \times \delta\left(\zeta \cos \theta+\zeta_{1} \cos \theta_{1}-\zeta_{2} \cos \theta_{2}-\zeta_{3} \cos \theta_{3}\right) \\
& \times \delta\left(\zeta \sin \theta+\zeta_{1} \sin \theta_{1}-\zeta_{2} \sin \theta_{2}-\zeta_{3} \sin \theta_{3}\right) \\
& \times \delta\left(\sqrt{\zeta}+\sqrt{\zeta}_{1}-\sqrt{\zeta}_{2}-\sqrt{\zeta}_{3}\right) \\
& \times\left[P_{\beta}\left(\zeta_{1}, \theta_{1}\right) P_{\beta}\left(\zeta_{2}, \theta_{2}\right) P_{\beta}\left(\zeta_{3}, \theta_{3}\right)\right. \\
& +P_{\beta}(\zeta, \theta) P_{\beta}\left(\zeta_{2}, \theta_{2}\right) P_{\beta}\left(\zeta_{3}, \theta_{3}\right) \\
& -P_{\beta}(\zeta, \theta) P_{\beta}\left(\zeta_{1}, \theta_{1}\right) P_{\beta}\left(\zeta_{2}, \theta_{2}\right) \\
& \left.-P_{\beta}(\zeta, \theta) P_{\beta}\left(\zeta_{1}, \theta_{1}\right) P_{\beta}\left(\zeta_{3}, \theta_{3}\right)\right] \\
& \times \zeta_{1} \zeta_{2} \zeta_{3} d \zeta_{1} d \zeta_{2} d \zeta_{3} d \theta_{1} d \theta_{2} d \theta_{3}
\end{aligned}
$$

Similarly to the previous case (see Eq. 94) define:

$$
\begin{gathered}
A_{\beta}=\int \sqrt{\zeta} P_{\beta}(\zeta, \theta) \zeta d \zeta d \theta \\
B_{\beta}=\int \zeta P_{\beta}(\zeta, \theta) \zeta d \zeta d \theta .
\end{gathered}
$$

For mean frequency and energy one has

$$
\epsilon=u_{\chi} \chi^{p_{\chi}} ; \quad v=v_{\chi} \chi^{-q_{\chi}}
$$

where

$$
\begin{aligned}
q_{\chi}=\frac{2 p_{\chi}+1}{10}, & p_{\chi}=\frac{5 \beta-1}{2} ; \\
u_{\chi}=b^{5 / 2} A_{\beta} ; & v_{\chi}=u_{\chi}^{1 / 5} \frac{B_{\beta}}{A_{\beta}^{4 / 5}} .
\end{aligned}
$$

Quite similarly to the duration-limited case one can treat Eq. (102) in self-similar variables as stationary and homogeneous equation that describes equilibrium of nonlinear transfer (term $S_{n l}$ ) and a specific source term

$S_{i n}^{*}\left(P_{\beta}(\zeta)\right)=\frac{\cos \theta}{2 \sqrt{\zeta}}\left[\alpha P_{\beta}(\zeta)+\beta \zeta \frac{\partial P_{\beta}(\zeta)}{\partial \zeta}\right]$

The source term is linear in function $P_{\beta}$ and not small because it appears from strong effect of non-homogeneity. This balance equation gives a family of self-similar solutions. The higher order "non-conservative" approximation should be considered to specify parameters (self-similarity indexes) of these solutions quite similarly to the duration-limited case. Integrating Eq. (99) over $\kappa$ and $\theta$ one gets the balance

$$
\frac{1}{2} \int \frac{\cos \theta}{\Omega} \frac{\partial n}{\partial \chi} \kappa d \kappa d \theta=\int \widetilde{S}_{i n} \kappa d \kappa=Q_{\chi}(\chi)
$$

Here $Q_{\chi}$ is the total input of wave action - a net result of wind forcing and wave breaking dissipation. The condition (Eq. 103) is consistent with self-similar forms of solutions (Eq. 100) if the total input is a power-like function of fetch $\chi$. The corresponding exponents are obtained easily from re-scaling properties of the collision integral $S_{n l}$ (Eq. 29)

$Q_{\chi} \sim \chi^{\frac{7 \beta-3}{2}}$
For total growth of wave action one gets

$N_{t o t}=\int n(\boldsymbol{k}) d \boldsymbol{k} \sim \chi^{\alpha-2 \beta} \sim \chi^{3 \beta-1 / 2}$

Basic cases can be specified for particular sets of exponents quite similarly to the duration-limited case. First, the swell case when total wave action is constant gives (see Eq. 105)

$r_{\chi}=\alpha-2 \beta=0 ; \quad p_{\chi}=-1 / 12$

$q_{\chi}=1 / 12 ; \quad T=p_{\chi} / 2 q_{\chi}=-1 / 2$

The case of constant total input of wave action $Q_{\chi}=$ const; $\beta=3 / 7$ (see Eq. 104) has been studied by Zakharov and Zaslavsky (1983) and by Glazman (1994). The corresponding exponents are

$$
\begin{aligned}
& r_{\chi}=11 / 14 ; \quad p_{\chi}=4 / 7 ; \\
& q_{\chi}=3 / 14 ; \quad T=p_{\chi} / 2 q_{\chi}=4 / 3
\end{aligned}
$$

While the wave action input is constant the total wave action for the case (Eq. 107) grows slower than linear function of fetch

$N_{t o t} \sim \chi^{\alpha-2 \beta} \sim \chi^{11 / 14}$

The exponents (Eqs. 106 and 107) differ slightly from ones for the duration-limited case (compare Table 2). This difference can be explained easily by the effect of wave spectra downshift in spatially nonuniform wave field: wave input is damped by dispersion of longer waves propagating offshore.

The case of linear growth of total wave action can be associated with constant input of wave energy $\left(\bar{\omega} Q_{\chi}(\chi)=\right.$ const, $\left.r_{\chi}=1\right)$

$$
\begin{aligned}
r_{\chi}=1 ; & p_{\chi}=3 / 4 ; \\
q_{\chi}=1 / 4 ; & T=p_{\chi} / 2 q_{\chi}=3 / 2
\end{aligned}
$$

The special cases of fetch-limited growth are summarized in Table 4. In spite of quantitative difference of exponents (compare Table 2) there is a key common feature of duration-limited and fetch-limited cases: the exponents for constant wave action and wave energy inputs make left- and right-hand sides of the corresponding balance Eqs. (89 and 102) vanishing separately for the stationary isotropic powerlike Kolmogorov-Zakharov solutions. The KZ solutions split the effect of non-homogeneity and nonlinear transfer in Eq. (102) in self-similar variables and, thus, can be treated as asymptotic solutions of the self-similar family (Eq. 100) at infinitely long fetches $\chi \rightarrow \infty$.

Similarly to the duration-limited case one can show that self-similar solutions of this section are governed by inverse cascades of wave action and energy. In the special case of swell inverse and direct cascades of energy coexist. The exponents of dependencies of fluxes and of collision integral on fetch are given in Table 5 (compare Table 3 ). 
Table 4. Exponents of self-similar solutions in fetch-limited case.

\begin{tabular}{ccccccl}
\hline$r_{\chi}$ & $\alpha=\frac{5 r_{\chi}+1}{3}$ & $\beta=\frac{2 r_{\chi}+1}{6}$ & $p_{\chi}=\frac{10 r_{\chi}-1}{12}$ & $q_{\chi}=\frac{2 r_{\chi}+1}{12}$ & $T$ & \multirow{2}{*}{ Regime } \\
\hline 0 & $1 / 3$ & $1 / 6$ & $-1 / 12$ & $1 / 12$ & $-1 / 2$ & Swell \\
$11 / 14$ & $23 / 14$ & $3 / 7$ & $4 / 7$ & $3 / 14$ & $4 / 3$ & Constant wave action input \\
1 & 2 & $1 / 2$ & $3 / 4$ & $1 / 4$ & $3 / 2$ & Constant wave energy input \\
\hline
\end{tabular}

Table 5. Exponents of fluxes evolution for self-similar solutions in fetch-limited case.

\begin{tabular}{ccccl}
\hline$r_{\chi}$ & $S_{n l}(\boldsymbol{k}, \chi) \sim \chi^{s} S_{n l}(\zeta)$ & $Q(\boldsymbol{k}, \chi) \sim \chi^{s_{q}} Q(\zeta)$ & $P(\boldsymbol{k}, \chi) \sim \chi^{s_{p}} P(\zeta)$ & Regime \\
\hline 0 & $s=\left(22 r_{\chi}-7\right) / 12$ & $s_{q}=\left(14 r_{\chi}-11\right) / 12$ & $s_{p}=\left(r_{\chi}-1\right)$ & \\
$11 / 14$ & $-7 / 12$ & $-11 / 12$ & -1 & Swell \\
1 & $6 / 7$ & 0 & $-3 / 14$ & Constant wave action input \\
\hline
\end{tabular}

5.5 Self-similarity and experimental exponents of windwave growth

Power-like approximations of parameters of wind-wave growth are of common use in wind-wave studies (e.g. Babanin and Soloviev, 1998). The question is: "How the experimental approximations can be related to our theoretical findings?" For total energy and mean frequency $\bar{\omega}$ one has

$E_{\text {tot }} \sim \bar{\omega}^{-p / q}$

We omit subscripts for $p_{\tau}, q_{\tau}, p_{\chi}, q_{\chi}$ here to show independence of the trivial relation on conditions of generation (duration- or fetch-limited growth). For JONSWAP spectra (Eq. 37) the exponent of energy growth $\kappa_{\alpha}$ can be determined easily

$\kappa_{\alpha}=5-r / q=4-p / q=4-2 T$

Surprisingly, the exponents $\kappa_{\alpha}$ for basic regimes presented in Tables 2 and 4 are the same in time-limited and fetchlimited cases: $\kappa_{\alpha}=1$ for constant wave energy input, i.e. exactly Toba's law (Toba, 1973) and $\kappa_{\alpha}=4 / 3$ for constant wave action input.

Our exercises with exponents $\kappa_{\alpha}, p, q$ look like a juggling by notations with no reference to the Hasselmann equation. In fact, very strong hypotheses underly this juggling: the self-similarity of the asymptotic solutions for the Hasselmann equation and self-similar form of JONSWAP spectrum (see Sect. 3.1). Real wind-wave spectra are, evidently, not self-similar. They can manifest their self-similarity features partially only, say, for certain range of directions and wave frequencies where nonlinearity dominates as compared to input and dissipation. Beyond this range the wave spectra are affected heavily by details of wind input and dissipation, by initial and boundary conditions etc. The magnitudes of the non-self-similar fraction of wave field are likely relatively small but total energy content and mean wave frequency can differ significantly from predictions of self-similar behaviour. In other words, the exponent of spectral growth $\kappa_{\alpha}$
(Eq. 39) can be determined by different methods: first, in terms of total energy and mean frequency (see Eq. 109), i.e. taking into account both self-similar and "background" fractions of wind-wave field and, second, in terms of local characteristics of wind-wave growth: peak frequency exponent (downshift exponent) $\beta$ and spectral peak magnitude exponent $\alpha$. In terms of these two quantities Eq. (109) can be rewritten as

$\kappa_{\alpha}=9-2 \alpha / \beta$

This presentation is valid for both duration and fetch-limited growth and can be useful as an alternative definition of $\kappa_{\alpha}$ in analysis of numerical results. Comparing exponents determined from "global" characteristics of wind-wave field (mean frequency and total energy - Eq. 109) and from "local" exponents of spectral peak evolution (frequency and amplitude of spectral peak - Eq. 110) one can quantify selfsimilarity features of wave spectra. This gives us a solid basis for analysis of our numerical results.

\section{Numerical solutions for the kinetic equation}

In this section we present results of numerical solutions of the kinetic equation for the case of duration limited growth (Eq. 6). Details of numerical algorithm used in this paper have been published in many papers (e.g. Webb, 1978; Resio and Perrie, 1991; Pushkarev et al., 2003). The code based on this algorithm has been developed by Tracy and Resio (1982) and modified recently by Pushkarev (Pushkarev et al., 2003). Extensive numerical studies performed with this code (Pushkarev et al., 2003; Badulin et al., 2002) showed its adequate accuracy and stability in a wide range of parameters of wave field and external forcing. In this paper we give just a brief overview of features of the algorithm and the numerical approach.

The numerical study is based on the algorithm of calculation of the collision integral $S_{n l}$ proposed by Webb (1978). 
The resonant subspace is parameterized by a series of resonant curves - locii with a fixed pairs of wavevectors $\boldsymbol{k}_{1}$ and $\boldsymbol{k}_{3}$ in conditions of resonance (Eq. 1). Alternative approaches (e.g. Komatsu and Masuda, 1996; Lavrenov, 2003) perform an analytical integration of $S_{n l}$ in order to reduce dimension of integration domain. It leads to a complicated form of the resulting domain and, hence, to additional problems with accuracy and time of calculations.

In a majority of our numerical experiments the following parameters have been used:

1. Range of wave scales $-0.02-2 \mathrm{~Hz}$ (wavelengths approximately $40 \mathrm{~cm}-4 \mathrm{~km}$ );

2. Grid is regular in angle with $10^{\circ}$ step and logarithmic in frequency with increment 1.068 (71 points in frequency);

3. 30 points are taken to calculate contour integrals along each locus;

4. Harmonics of essentially different scales are not taken into account, i.e. quadruplets with frequency ratio more than 3 (factor 9 for wavelength) are ignored. Additionally, quadruplets with magnitude of kernels below a certain small threshold are ignored. Effectively, about 50000 quadruplets are taken into account in $S_{n l}$ calculations.

An explicit integration scheme with adaptive time step has been used. All the parameters given above are consistent with ones recommended by Komatsu and Masuda (1996). Comparison with their results of the kinetic equation solutions showed reasonable quantitative agreement for mean energy, frequency and for features of spectral distributions. The exact coincidence could not be achieved because some parameters of their numerical runs were not specified in the paper explicitly. The results of the comparison are not discussed here and will be presented in a separate paper.

Calculations have been made for water depth $2000 \mathrm{~m}$ and wind speeds from 5 to $30 \mathrm{~m} \cdot \mathrm{s}^{-1}$. High-frequency cut-off of wave generation was at $1 \mathrm{~Hz}$ or as it is required by the conventional parameterizations considered in Sect. 3 of the paper. Strong dissipation is assumed for frequencies higher than $1 \mathrm{~Hz}$.

Time of spectra evolution was generally limited by $10^{5} \mathrm{~s}$ (slightly more than 1 day). In cases of slow evolution (low winds, wave swell) this time was extended up to $10^{6} \mathrm{~s}$. Calculation time was typically one-to-one with time of evolution (CPU AMD Barton 3000, Fortran77 under Linux).

\section{1 "Academic" series}

The justification of the theoretical background given above remains our red line. In this section we start with "academic" numerical experiments. The conditions of generation in these experiments can be considered as unrealistic but they allow one to focus on fundamental qualitative and quantitative features of the problem. The idea of the academic runs is not new and was exploited, for example, for numerical estimates of fundamental Kolmogorov's constants (Pushkarev et al., 2003; Lavrenov et al., 2002). The features of self-similarity are reproduced in our academic experiments perfectly well as it will be seen below. They give a very good reference to study "realistic" cases where the self-similarity is contaminated by effects of strong anisotropy and wave forcing in a wide frequency domain.

In this section we present a series of numerical runs that is specially designed to detail self-similarity properties of the kinetic equation solutions. Starting with very low "white noise" as initial conditions we put a time-dependent source function into high-frequency range in order to reproduce inverse cascade regimes corresponding to the family of selfsimilar solutions considered above. In this setup one can control easily the total wave action flux as a power-like function of time in full accordance with the asymptotical procedure of previous section.

\subsection{1 "Academic" series with isotropic source function}

We start with absolutely unrealistic problem of isotropic source function in order to relate our numerical results with the Kolmogorov-Zakharov solutions for direct and inverse cascades which are essentially isotropic. This unrealistic setup has a long story, recently it has been used in numerical studies of the direct cascade (Pushkarev et al., 2003; Lavrenov et al., 2002). The idea is very simple: to reproduce key features of the theoretical model in numerical experiments, first of all, to leave maximal space for the so-called inertial frequency range where the nonlinear transfer is the only physical mechanism of wave evolution.

The initial conditions in all the experiments (excluding the case of zero wave input - swell ) corresponded to very low initial wave amplitudes - the significant height was approximately $1 \mathrm{~cm}$. The source function in the experiments has been set up as a time-dependent function

$S_{i n}=\beta(\boldsymbol{k}) N(\boldsymbol{k})=\beta_{0}\left(t / t_{0}\right)^{R-1} N(\boldsymbol{k})$

in a frequency range $1-1.5 \mathrm{~Hz}$. The constant $\beta_{0}$ was chosen sufficiently high to provide essential evolution for reasonable time of calculations. In fact, all these "unrealistic" experiments can be related to realistic temporal and spectral scales of wind wave evolution. For example, the significant wave heights up to $10 \mathrm{~m}$ were reached for very reasonable physical time 1 day in these runs. Frankly speaking, the only "unrealistic" feature of these experiments is strong localization of wave input domain in frequency.

Calculations have been carried out for a series of exponents $R$ in the range $1 / 2 \leq R \leq 4 / 3$ that approximately corresponds to "acceptable" rates of wave action growth for the self-similar solutions (see Sect. 5.2 and Eq. 93) and for the special case of wave swell. The most important advantage of the source function is seen in Fig. 11. Very large inertial interval has been provided thanks to very narrow domain of generation and strong relaxation of solutions to some "inherent" state beyond the domain. A good agreement with 

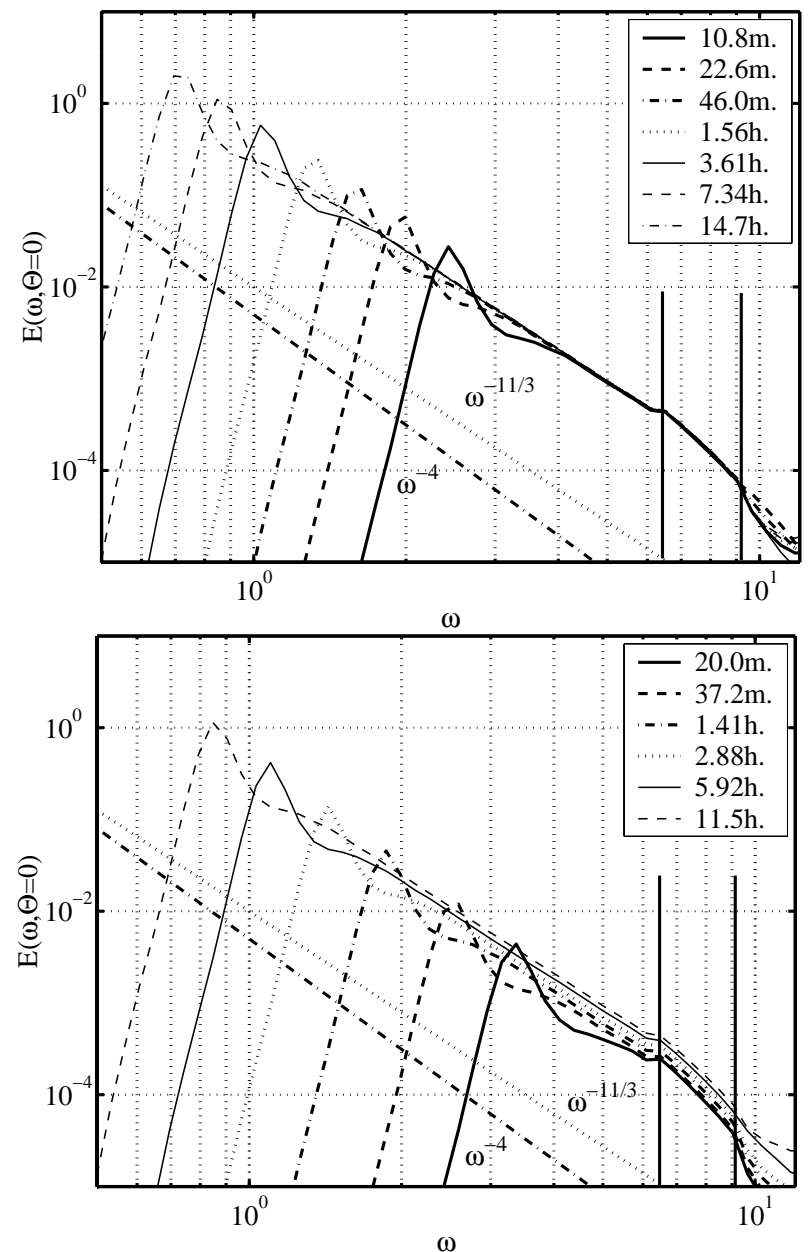

Fig. 11. Isotropic solutions in "academic" runs for different values of exponent $R$ (see Eq. 111). Generation domain limits are shown by vertical solid lines. Asymptotics for inverse (dotted line) and direct (dash-dotted) cascades are shown. Top $-R=1$, bottom $R=4 / 3$.

Kolmogorov's inverse cascade solution was found in terms of slopes of the spectra in the inertial interval (asymptotes $\omega^{-11 / 3}$ are shown by dotted line in Fig. 11) in all the range of parameter $R$.

The source function (Eq. 111) provides very good fit to power-like wave action input and makes possible to extract the self-similar dependencies from numerical solutions. The self-similarity of the solutions is illustrated quite well by Fig. 11 where solutions taken at approximately log-spaced times appear to be log-spaced both in amplitudes and in frequencies. Figure 12 gives quantitative analysis of the selfsimilarity features for the case of constant wave action input $R=1$. First, mean and peak frequencies show powerlike dependencies on time with exponents which are very close to the theoretical exponent $\beta / 2=3 / 11$ (top panel). Bottom panel in Fig. 12 presents the shape function $U_{\beta}(\xi)$ (see Eq. 88) extracted from numerical solutions at different times.

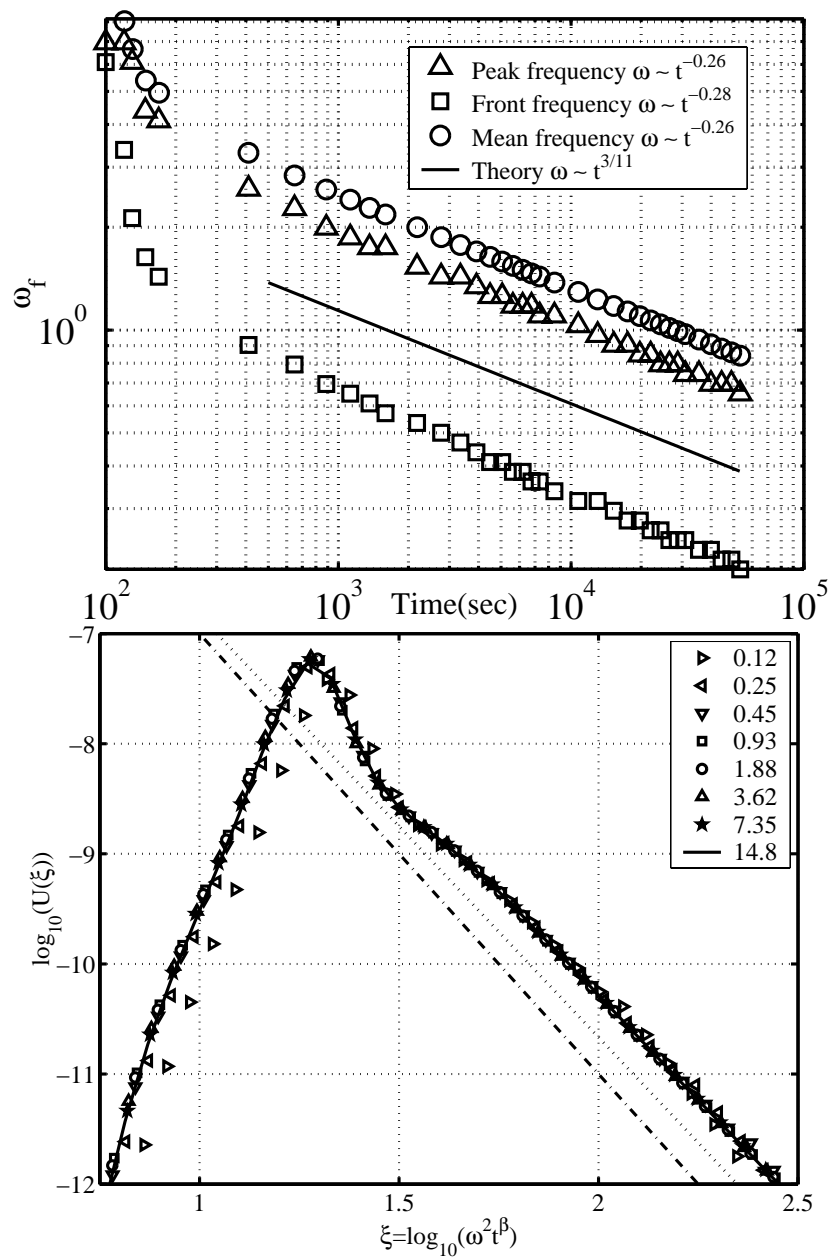

Fig. 12. Self-similarity of the kinetic equation solutions for the "academic" case at $R=1$. Top - dependence of mean (circles), peak (triangles) and front (squares - positive maximum of spectral slope) frequencies on time. The exponents of downshift $\beta$ are close to the theoretical value $\beta / 2=3 / 11$. Bottom - function $U(\xi)\left(\xi=\omega^{2} t^{6 / 11}\right)$ extracted from the numerical solutions at different times (see legend, in hours). Slopes $\xi^{-4}$ and $\xi^{-23 / 6}$ for direct (dash-dotted) and inverse (dotted) cascades are shown

The theoretical values $\alpha=23 / 11, \beta=6 / 11$ were taken for the corresponding transformation. One can see relaxation to a permanent form for extremely short time: no visible difference of solutions is seen for $t \geq 0.45 \mathrm{~h}$.

All the experiments with different wave input rates $R$ show the same strong tendency to self-similar behaviour. A remarkable result of our study: Shape function $U_{\beta}(\xi)$ does not depend (more conservatively, does not depend within the accuracy of our numerical approach) on index of self-similarity $\beta$. This result is illustrated in top Fig. 13. $U_{\beta}(\xi)$ normalized on their peak magnitudes were traced as functions of the normalized argument - non-dimensional wave frequency $|\xi|=2 \log \left(\omega / \omega_{p}\right)$. For different parameters of wave input $R$ (shown in legend) these functions are amazingly close to each other. 

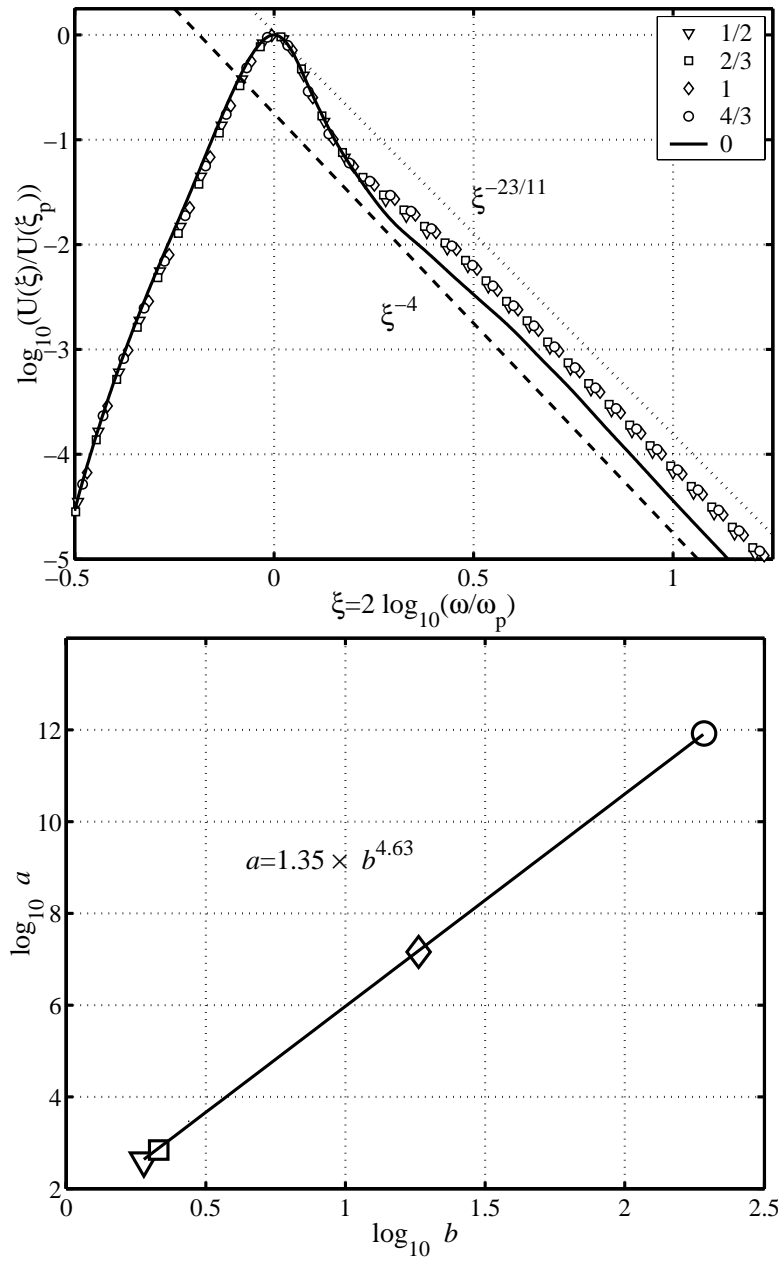

Fig. 13. Shape functions $U_{\beta}$ for different parameters of wave input $R$ (top). The solutions for different rates $R$ are shown by different symbols (in legend). The swell case is given by solid line. Bottom - the scaling of the solution peaks and the peak positions. Fitting formula and the corresponding fitting line are given.

The high-frequency tails are found to be very close to the inverse cascade Kolmogorov's exponent $(-23 / 6)$ for runs with wave input. In fact, the difference between exponents of direct and of inverse cascades $(-4)$ and $(-23 / 6)$ is rather small and the above conclusion seems speculative in view of the problem of correct estimates of these exponents within the numerical approach. Nevertheless, a clear evidence that the spectral slopes are less than one of the direct cascade Kolmogorov solution $\xi^{-4}$ can be acquired from Fig. 11. For $R=1$ (top) the solution is stationary in the domain of generation while for $R=4 / 3$ (bottom) the solution is growing with time in this frequency range. The solution in the generation domain can be estimated from the self-similar dependence (Eq. 89) as

$N\left(\boldsymbol{k}_{\text {input }}\right) \sim t^{\alpha-\kappa_{\beta} \beta}$

where $\kappa_{\beta}$ is exponent of the solution power-like tail. Thus, generally, $N\left(\boldsymbol{k}_{\text {input }}\right)$ depends on time if $\kappa_{\beta}$ does not fit the proper relations. The stationarity of generation domain for $R=1$ means that $\kappa_{\beta}=\alpha / \beta=23 / 6$. In bottom panel $N\left(\boldsymbol{k}_{\text {input }}\right)$ is definitely growing with time, that is, $\kappa_{\beta}<\alpha / \beta=4$.

Unlike the case of wave input the swell solution has highfrequency asymptotics visibly closer to the direct cascade asymptotics $\xi^{-4}$. This difference can be treated as a key difference of regimes governing the solutions: in case of wave input one has a purely inverse cascading in the domain of self-similarity while swell corresponds to a hybrid regime when domains of direct and inverse cascades coexist.

The universality of $U_{\beta}$ (quasi-universality - weak dependence on self-similarity index $\beta$ ) allows one to verify easily other important property of the self-similar solutions the relation between wave scales and spectra amplitudes (Eq. 90). Strictly speaking, this property should be checked for fixed exponent of wave input by changing initial conditions or rate of wave growth $\beta_{0}$ (Eq. 111). Bottom panel of Fig. 13 shows fairly well validity of scaling $a \sim b^{19 / 4}$ where parameters $a$ and $b$ have been estimated from characteristics of spectral peaks for different exponents $R$ of wave input. A small deviation of the power-like fit exponent in bottom Fig. $13(4.63<19 / 4)$ can be considered as a measure of quasi-universality of function $U_{\beta}(\xi)$. Thus, the scaling (Eq. 90) can be considered as universal one irrespectively to the particular regime of self-similar evolution.

6.1.2 Anisotropic self-similar solutions in "academic" numerical experiments

The kinetic equation admits of anisotropic self-similar solutions as it was pointed out in Sect. 5. Two evident questions arise:

- Do these anisotropic solutions emerge from arbitrary initial data?

- What are features of these solutions as compared to isotropic solutions?

Anisotropic source function for the series has been set up similarly to Eq. (111) in a semi-ring $\pi / 2<\theta<-\pi / 2$

$S_{\text {in }}= \begin{cases}\beta_{0} \cos \theta\left(t / t_{0}\right)^{R-1} N(\boldsymbol{k}), & \pi / 2<\theta<-\pi / 2 \\ 0, & \text { otherwise }\end{cases}$

The strong tendency to the self-similar behavior has been demonstrated for the same parameter set $1 / 2 \leq R \leq 4 / 3$ as for isotropic "academic" runs.

Figure 14 shows tendency of the numerical solution to self-similar behaviour for different angles at $R=1$. The exponent of total wave action growth appears slightly lower ( $r=0.97$ ) than in the isotropic case when $R=r=1$. This little difference is dealing with anisotropy when a fraction of wave input "leaks" to periphery of the self-similar "core" of solution. In fact, this negligible, at the first glance, effect results in rather strong qualitative and quantitative consequences. The leakage to domains where amplitudes of the solution and the generation rates are weak leads to formation of non-self-similar background. The exponent of total 

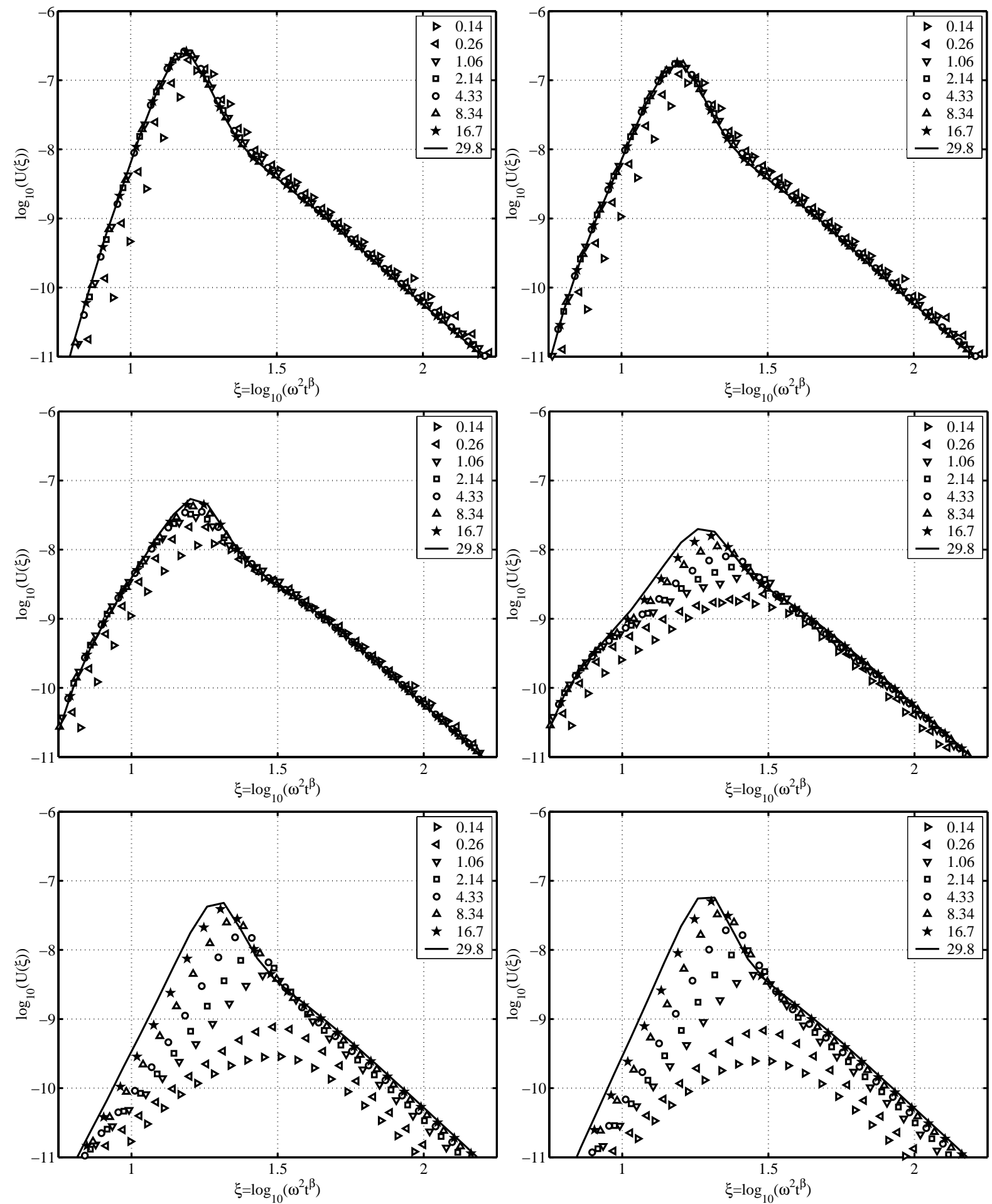

Fig. 14. Tendency to self-similar asymptotics for different angles to wind direction $0^{\circ}, 30^{\circ}, 60^{\circ}, 90^{\circ}, 150^{\circ}, 180^{\circ}$, the "academic" anisotropic run with $R=1$ (total wave action growth rate $r=0.97$ ). The exponent $r_{\exp }=\alpha-2 \beta=0.89$ determined from exponents of the solution peak growth $\alpha$ and $\beta$ was used to extract the self-similar dependence (see comments in Sect. 5.5).

wave action growth $r$ becomes inadequate to the self-similar "core" of the solution. In this case, exponents of local growth can be more relevant to the problem. In Fig. 14 the exponents $\alpha$ and $\beta$ of the self-similar solution (Eq. 88) have been deter- mined by tracing peak frequency and peak magnitude and the corresponding parameter $r_{\text {exp }}$ has been determined from evident relation $r_{\text {exp }}=\alpha-2 \beta$ given by Eq. (92). It gave essentially lower value $r_{\text {exp }}=0.89$ for the case $R=1$. Evidently, 

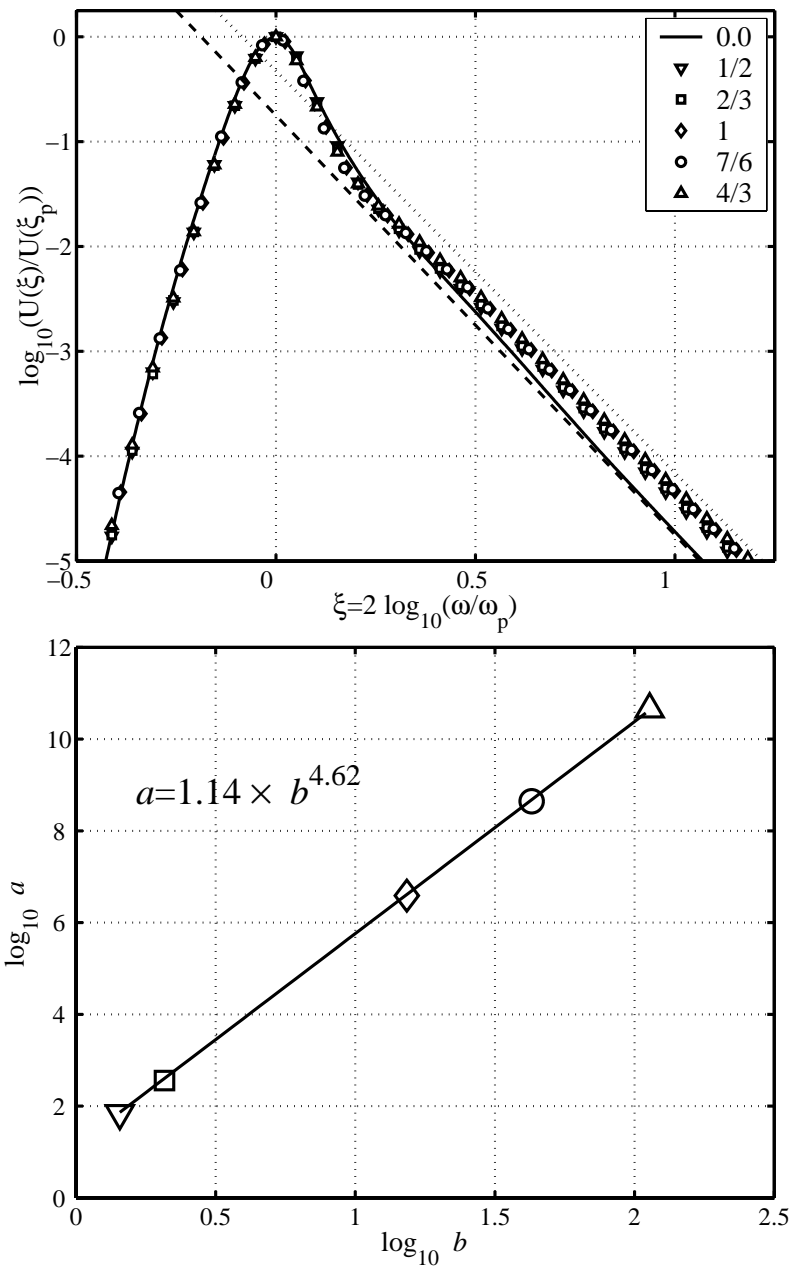

Fig. 15. The same as in Fig. 13 for anisotropic academic numerical solutions.

the non-self-similar background exists in the isotropic experiments as well, for example, in low-frequency domain which is not affected by the self-similar "core" but the effect is incomparably smaller than in the anisotropic case.

We see again an illustration of the problem of "magic circle": self-similarity requires strong nonlinearity and, in its turn, the strong nonlinearity requires strong wave input. In anisotropic case we always have a situation when nonlinearity is not strong enough to provide pronounced self-similarity features. It can be seen in Fig. 14 for oblique directions. The strongest tendency to a permanent shape function $U_{\beta}(\xi)$ extracted from the numerical solution is observed for downwind direction where the wave pumping is maximal: the solution for $1 \mathrm{~h}$, actually, fits solutions for larger times remarkably well, i.e. it is very close to a limiting function $U_{\beta}$ (if this limit exists). The tendency to a limiting shape is slower for oblique directions, but, unexpectedly, the tendency for upwind direction appears more pronounced than for the direction $90^{\circ}$. The latter can be explained by stronger nonlinear transfer from maximal down-wind components to up-wind ones.
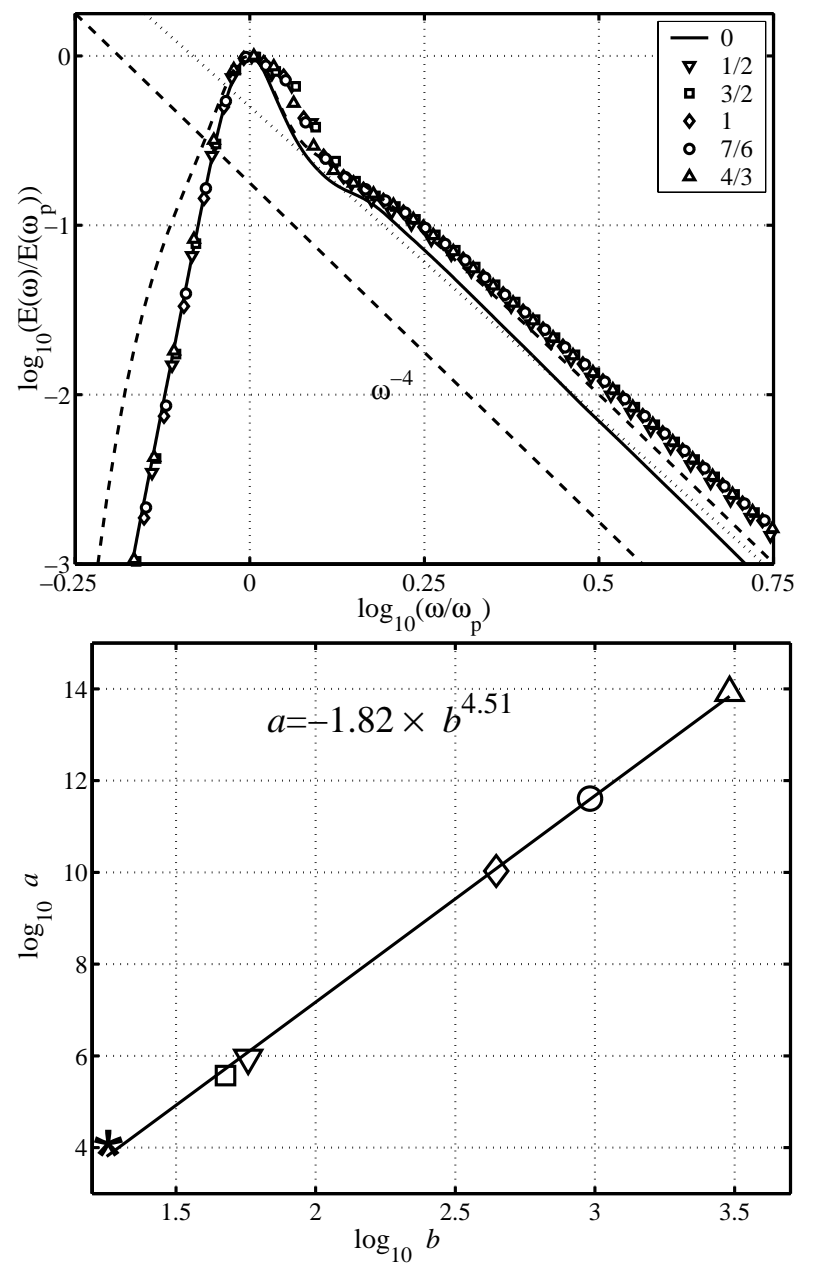

Fig. 16. The same as in previous figure for non-dimensional frequency spectra $E(\omega) / E\left(\omega_{p}\right)$. The JONSWAP spectrum for the standard peakedness $\gamma=3.3$ is shown by dashed curve. The dependence of the solutions parameters $a$ and $b$ is presented in bottom panel, swell scaling is given by $\star$.

Looking for functions $U_{\beta}(\xi)$ of the anisotropic solutions in Fig. 15 we find differences with isotropic solutions of the previous section. First, the low-frequency front is essentially steeper for the anisotropic solutions. Secondly, highfrequency spectral slopes show a weak dispersion. This question is to be studied more carefully with more accurate numerical approach. At the moment, one can conclude that the qualitative behaviour is well within the theoretical prediction: for lower wave input exponents (small $R$ ) the tails of solutions are closer to the direct cascade spectral slope (-4), while for higher values of $R$ the tails are more flat. The spectral tails of swell show definitely the direct cascade exponents $(-4)$.

The solutions' scaling for different $R$ manifests a remarkable agreement with the theoretical relation (Eq. 90) as it is seen in Fig. 15 (bottom). It should be noted that the scaling parameters of isotropic and anisotropic solutions appear very close to each other (compare multipliers in fitting formulas in bottom Figs. 13 and 15). 
An important result of the analysis of the "academic" anisotropic solutions has been found calculating normalized frequency spectra for different rates $R$ (Fig. 16). One can see closeness of the frequency spectra shapes to each other and, more, to the experimental JONSWAP spectrum (shown by dashed line). Thus, the self-similarity features of "academic" solutions are very close to ones of experimentally observed spectra of growing wind waves.

\section{2 "Real" wave input}

In this section we present results of numerical solutions for the kinetic Eq. (27) with parameterizations of wind-wave source function $S_{f}=S_{i n}+S_{\text {diss }}$ given by conventional formulas of Sect. 3. Taking the results of previous "academic" studies as a reference we show that details of the source function is of little importance for qualitative, and, more, for quantitative features of wind-wave evolution. It contradicts to the streamline of wind wave studies where tremendous efforts are mounted to describe details of wind-wave generation in different wave scales. In many cases it complicates essentially mathematical and physical approaches but give no gain for the problem understanding and for practical needs of wind wave forecasting.

The critics of the attempts to solve the problem of windwave modelling by "tuning" source function is not a point of the section. We try to fix some "trigger" points in order to understand where such tuning can lead to physically important results and where we have to switch our attention to other ways of the problem solution.

In spite of great number of numerical studies of wind wave evolution within the kinetic equation there is no a substantial foundation for analysis of the results. We use the concept of wind-wave spectra self-similarity as such foundation.

We start with analysis of initial stage of wind-wave evolution when nonlinearity is relatively weak, wave spectra evolution is not self-similar and depends essentially on details of initial data and of the source function $S_{f}$. This stage is typically very short in time for realistic wind wave conditions. Further evolution of wind wave field can be described quite well by self-similar dependencies.

\subsubsection{Solutions at initial stages}

The solution of the kinetic equation at initial stages of wind wave evolution is accompanied by a number of difficulties. The effects of initial data and details of source function are very important at this stage. Figure 17 illustrates this remark. The growth rates are identical for both examples presented in the figure. In left panel the evolution starts from "white noise" with significant wave height approximately 10 $\mathrm{cm}$. These conditions correspond to Rayleigh-Jeans equilibrium, the collision integral is plain zero for the initial state and, thus, linear pumping dominates at small times. When the solution deviates from the equilibrium state far enough, the nonlinear transfer becomes important. The direct cascade tail appears in an explosive way in high frequencies and the inverse cascade mechanism forces the solution to propagate to low frequencies in a front-like manner.

Qualitatively the same evolution is seen for the case of right panel. The Pierson-Moskowitz spectrum for $U_{20}=5 \mathrm{~m} \cdot \mathrm{s}^{-1}$ is taken as an initial condition (significant wave height is approximately $28 \mathrm{~cm}$ ) and wind speed $U_{10}=20 \mathrm{~m} \cdot \mathrm{s}^{-1}$. Strong dissipation is introduced for frequencies higher than $1 \mathrm{~Hz}$. Note, that for these initial conditions the collision integral does not vanish as in the previous case. Additionally, 2.8 times higher initial wave amplitudes means potentially $2.8^{6} \approx 482$ times higher nonlinear transfer term or $2.8^{4} \approx 61.5$ higher ratio of the nonlinear term to linear wave input. This is why the solution evolves essentially faster than in the first case. Even though the evolution is faster the resulting levels of solutions in a quasi-stationary high-frequency range are approximately the same in both cases: the solution "forgets" the initial data in a very short time.

The validity of the kinetic equation for the examples is questionable. The solutions evolve very rapidly with a typical time scale of only few hundreds periods tending to some inherent form of solutions which scales of evolution are compatible with the kinetic description of wave field. Additionally to the conceptual problem of applicability of the kinetic equation at small times the fast evolution gives rise to computational problem when time step has to be very small to provide adequate accuracy and stability of calculations.

\subsubsection{Solutions at large time - Self-similarity of solutions}

The most impressive feature in a relatively short time of evolution is similarity of the solutions in a wide range of conditions of wave generation. Figure 18 demonstrates this observation for wind speeds $10 \mathrm{~m} \cdot \mathrm{s}^{-1}$ and $20 \mathrm{~m} \cdot \mathrm{s}^{-1}$. Equation (51) by Hsiao and Shemdin (1983) for wind-wave growth rate was used in both cases. Solutions are different for different physical times, but in terms of wave age parameter $g /\left(\omega_{p} U_{10}\right)$ these solutions are very close to each other. This can be seen for solutions at final times in top and bottom panels of Fig. 18 (curve 6 in top and curve 5 in bottom one): the peak values of these solutions are the same for the same (approximately) wave ages $g /\left(\omega_{p} U_{10}\right) \approx 1$ while physical times are essentially different - approximately 32 (left) and 16 (right) h. Solutions in Fig. 18 are log-spaced in time and show clearly power-like dependence of peak frequency on time in perfect agreement with our theoretical analysis of self-similar solutions (see Eq. 88). Additional argument for the weak turbulence theory is in spectral slopes which are close to the KolmogorovZakharov exponents.

Two-dimensional wave spectra in Figs. 19 and 20 show that the Kolmogorov's power-like dependencies and similarity features of solutions are essential for anisotropic solutions as well. Energy spectra contours are shown for two different wind speeds as functions of non-dimensional frequency $\tilde{\omega}=\omega U_{10} / g$ (see Fig.18). The corresponding wave ages are calculated for spectral peak frequency without interpolating in frequency grid (this is why wave ages are slightly different for pairs of contours for different wind speeds). Contour lev- 

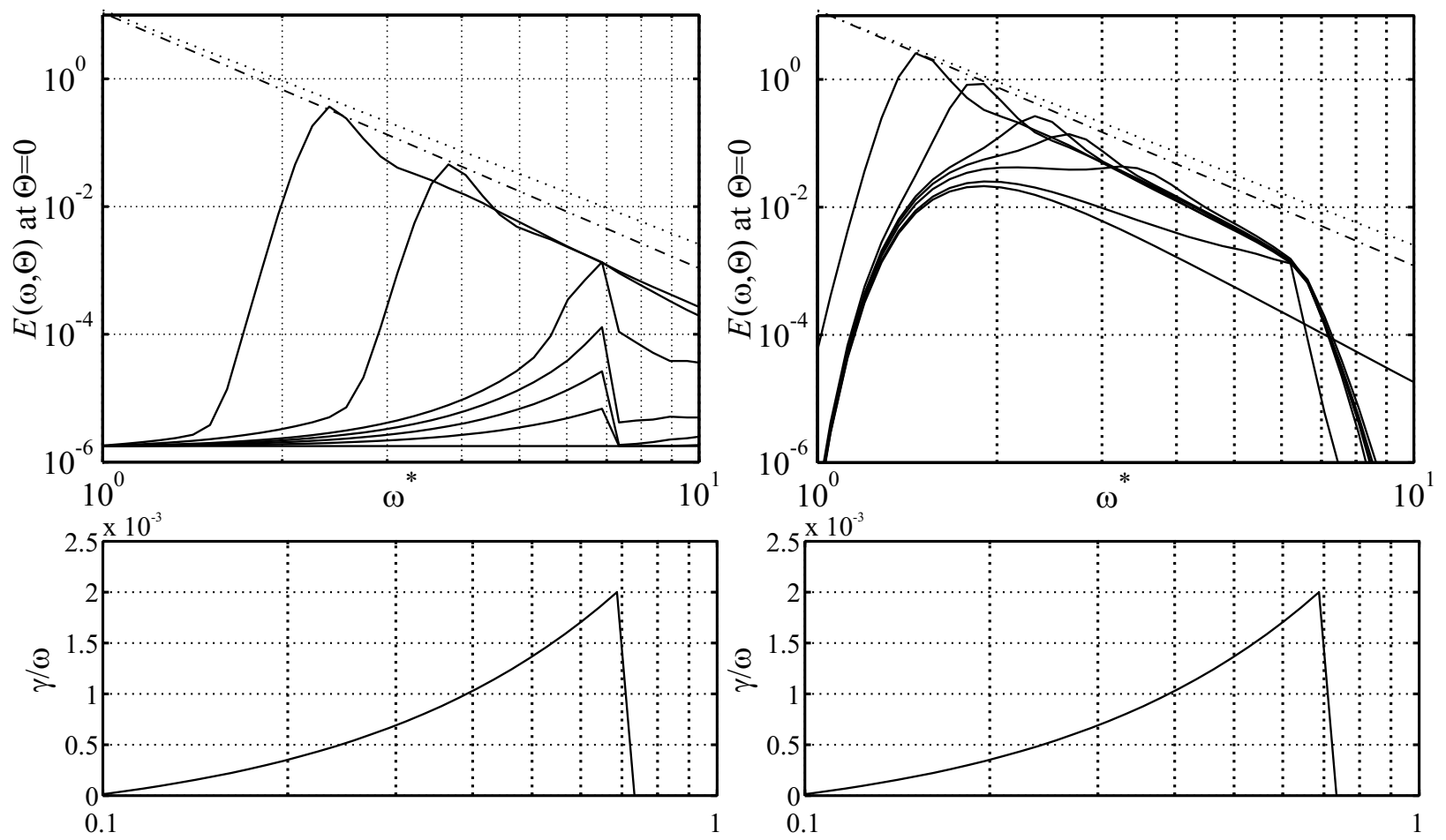

Fig. 17. Non-dimensional frequency spectra $E(\omega, 0)$ vs. non-dimensional frequency $\tilde{\omega}=\omega U_{10} / g$ for small times. Left column - initial condition is a "white noise" (equipartition) of energy $E(\omega)$ (significant wave height approximately $10 \mathrm{~cm}$ ). Input rate (bottom panel) is given by Snyder's et al. formula (Eq. 47) for wind speed $U_{10}=20 \mathrm{~m} \cdot \mathrm{s}^{-1}$, dissipation is absent. Right column - initial condition is the PiersonMoskowitz spectrum for wind speed $U=5 \mathrm{~m} \cdot \mathrm{s}^{-1}$ (significant wave height approximately $28 \mathrm{~cm}$ ). Input rate (bottom panel) is the same as in left column, strong dissipation is introduced for frequencies higher than $1 \mathrm{~Hz}$. Solutions for down-wind direction are given for a sequence of non-dimensional times $408(t=200 \mathrm{~s})$ ), 816, 1250, 1680, 3860, 7370 (physical time approximately $1 \mathrm{~h}$ ). Dotted and dashed lines correspond to inverse and direct cascade exponents $11 / 3$ and 4 (for frequency spectra of energy).
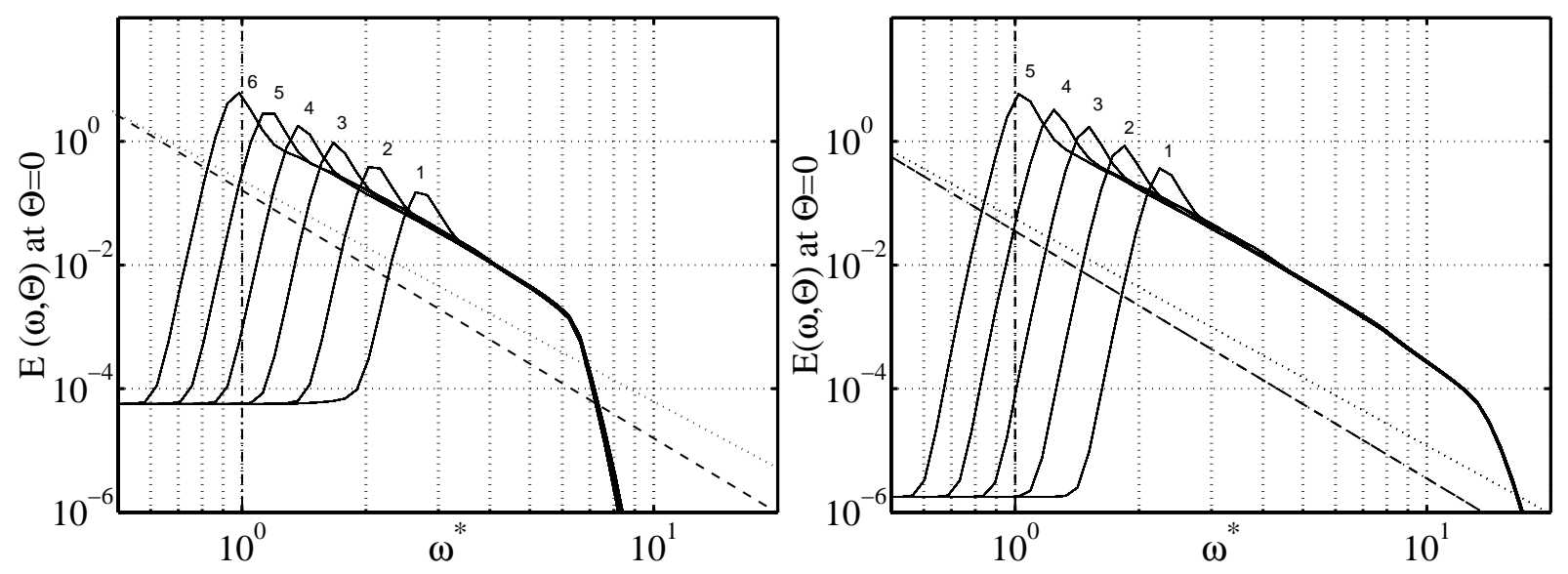

Fig. 18. Non-dimensional (Eq. 86) down-wind energy spectral density vs. non-dimensional frequency for two wind speeds $-10 \mathrm{~m} \cdot \mathrm{s}^{-1}$ (left) and $20 \mathrm{~m} \cdot \mathrm{s}^{-1}$ (right). Wave input is given by Hsiao and Shemdin (1983) (Eq. 51), strong dissipation is taken for $f>1 \mathrm{~Hz}$. Solutions are shown for the same set of log-spaced nondimensional times $\tilde{t}=g t / U_{10}: 1700$ (number 1); 3500 (2), 7000 (3), 14000 (4), 28000 (5), 59000 $\left(6\right.$, not shown for $\left.20 \mathrm{~m} \cdot \mathrm{s}^{-1}\right)$, i.e. for physical times approximately $0.5,1,2,4,8,16 \mathrm{~h}$ for the case $10 \mathrm{~m} \cdot \mathrm{s}^{-1}$. Asymptotes for direct (dash-dot, exponent (-4)) and inverse (dotted, $(-11 / 3)$ ) cascades are shown. Vertical dash-dotted lines correspond to wave age $g /\left(\omega U_{10}\right)=1$.

els are log-spaced and, thus, power-like dependence on wave frequency is clearly seen in these figures. Asymptotics of isotropic Kolmogorov's solutions are likely consistent with what we see in a wide range of angles $\pm 40^{\circ}$ near wind direction. Wave spectra for different wind speeds but for the same wave ages look very close to each other. Stress, that wave age is responsible for the scaling of these solutions but not physical time! Again we come back to the idea of self-similarity of these solutions. 
$\mathrm{T}=1.43 \mathrm{hrs} ; \mathrm{t}=5072 ; \omega=1.88$

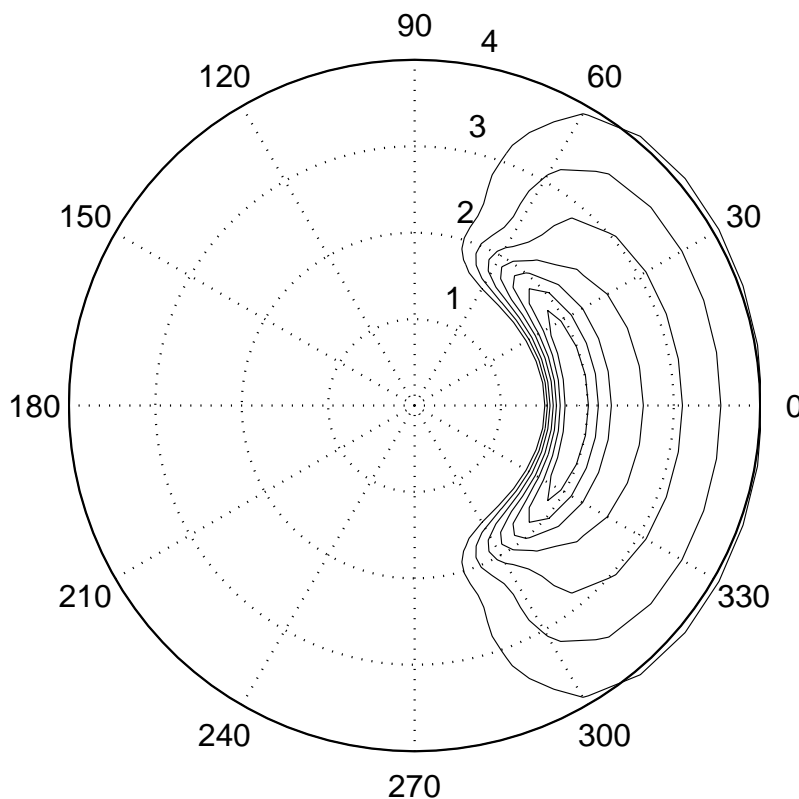

$\mathrm{T}=2.77 \mathrm{hrs} ; \mathrm{t}=9813 ; \omega=1.54$

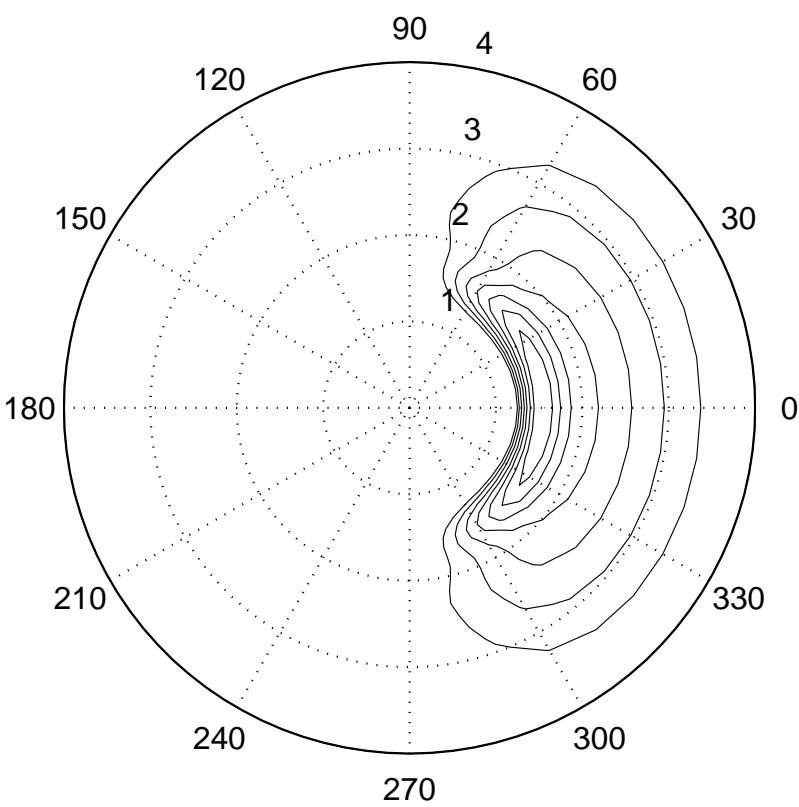

$\mathrm{T}=2.03 \mathrm{hrs} ; \mathrm{t}=3600 ; \omega=1.84$

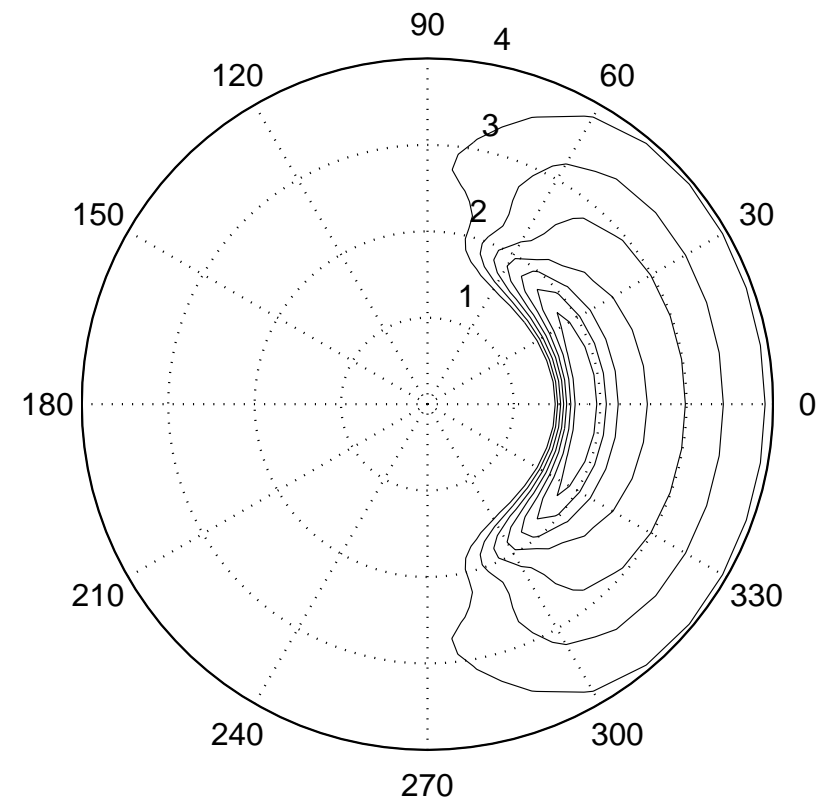

$\mathrm{T}=4.18 \mathrm{hrs} ; \mathrm{t}=7391 ; \omega=1.51$

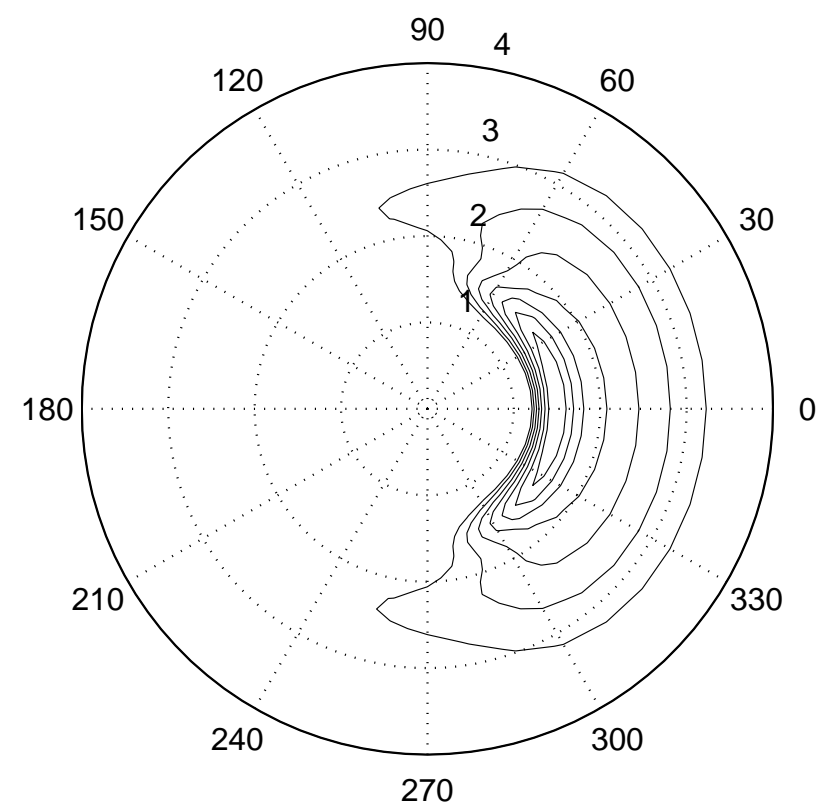

Fig. 19. Energy spectra for different inverse wave ages $\tilde{\omega}=\omega_{\text {peak }} U_{10} / g$ (shown in legends) as functions of nondimensional frequency $\tilde{\omega}=\omega U_{10} / g$, source function is given by Eq. 51 for wind speeds $10 \mathrm{~m} \cdot \mathrm{s}^{-1}$ (left column) and $20 \mathrm{~m} \cdot \mathrm{s}^{-1}$ (right column). Peak frequencies are calculated by interpolating between grid knots. Levels are normalized by peak values and log-spaced as $10^{-1 / 2}, \quad 10^{-3 / 4}, \quad 10^{-1}, \quad 10^{-5 / 4}, \quad 10^{-6 / 4}, \quad 10^{-7 / 4}$. Charts show impressive similarity of contours for spectral densities exceeding $10 \%$ of peak values.

Great difference of the solutions in left and right columns in Figs. 19 and 20 for large angles to the wind gives nothing but justification of our guideline on effect of dominating nonlinearity. The difference is great for the solutions peripheries where magnitudes are less than $3 \%$ of peak values. The small magnitudes mean dramatic (as magnitude in cube!) decrease of nonlinearity while linear terms of input and dissipation fall much slower. Existence of the non-self-similar background can contaminate links of our numerical results with theoretical models presented above. We faced this problem in the anisotropic academic runs but for "real" input it is complicated by a large domain of wave input.

Following the previous section approach one can extract shape function $U_{\beta}$. The question is: how to determine the corresponding index of self-similarity for a particular numerical solution? The parameter of wave action growth $r$ appears 
$\mathrm{T}=5.98 \mathrm{hrs} ; \mathrm{t}=21118 ; \omega=1.25$

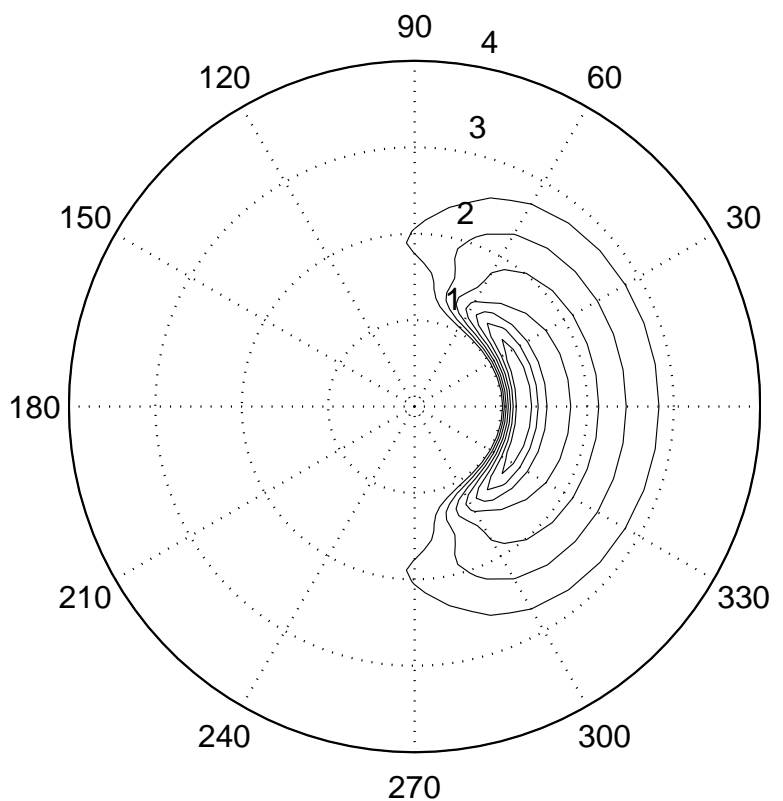

$\mathrm{T}=13.9 \mathrm{hrs} ; \mathrm{t}=49379 ; \omega=1.02$

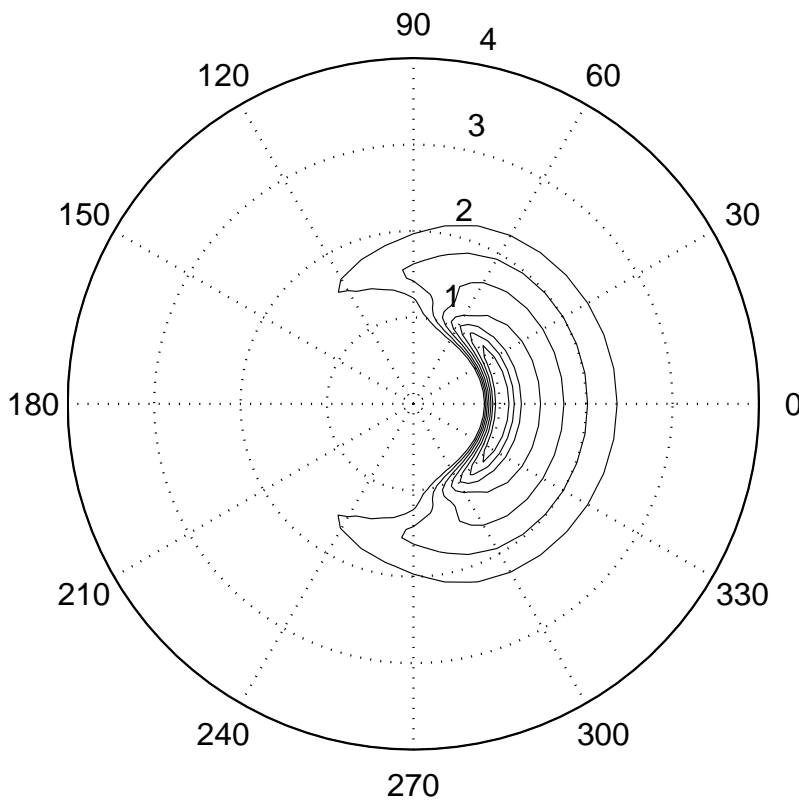

$\mathrm{T}=8.44 \mathrm{hrs} ; \mathrm{t}=14908 ; \omega=1.24$

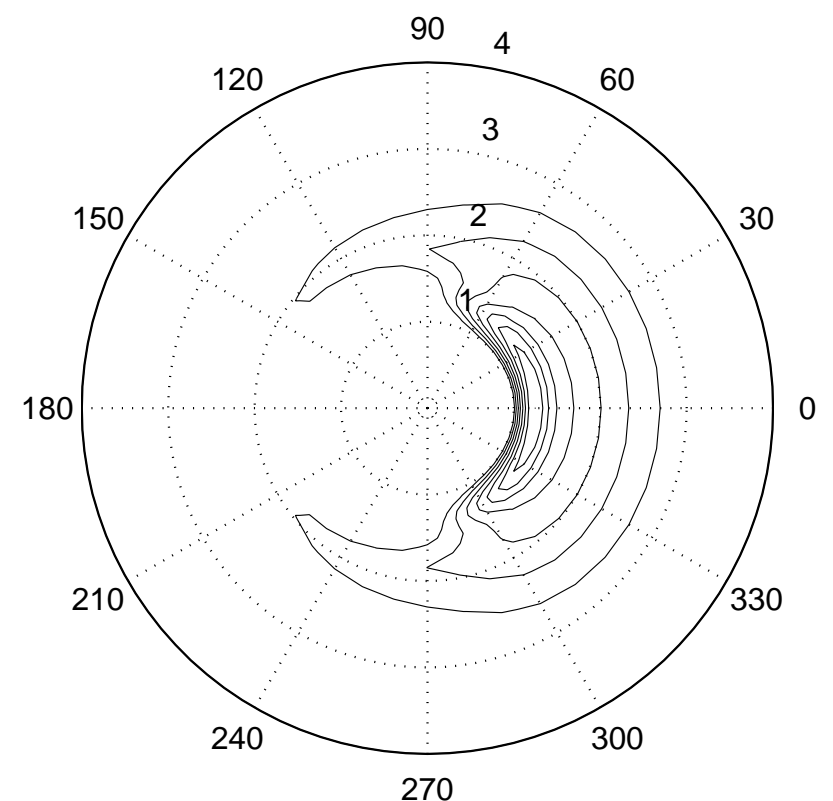

$\mathrm{T}=17.3 \mathrm{hrs} ; \mathrm{t}=30692 ; \omega=1.01$

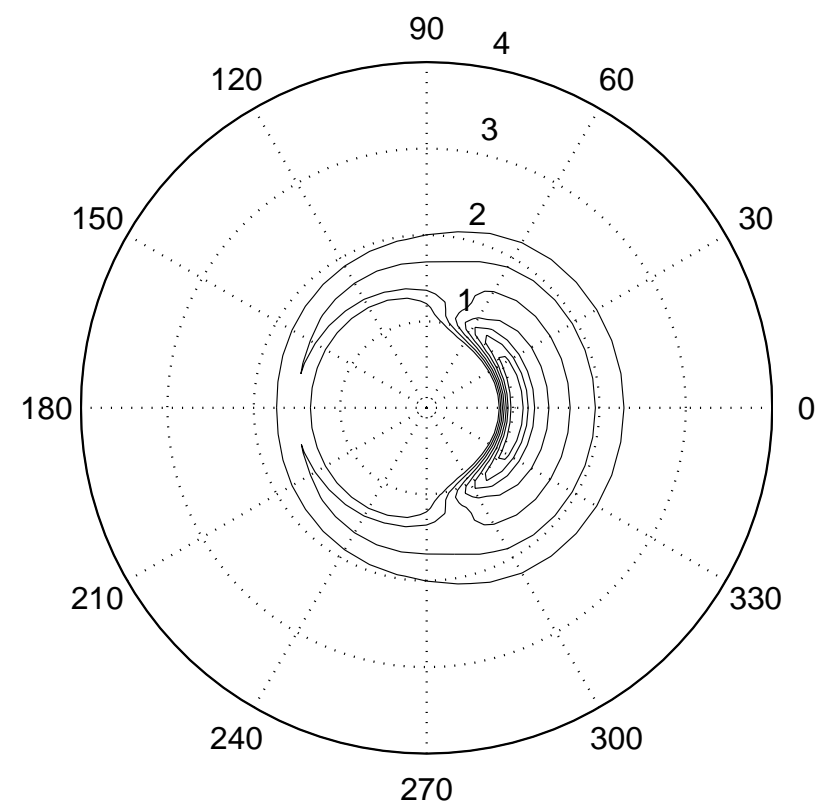

Fig. 20. Same as in previous figure for small inverse wave ages (shown in legend).

to be not adequate in this case because of the contaminating effect of essentially non-self-similar background. An alternative way to specify the self-similarity parameter is to extract it from local features of the solution (see notes of Sect. 5.5) as it was employed for academic runs using the exponents of the solution peak growth $\alpha$ and $\beta$.

Results are presented in Fig. 21 for different directions relatively to the wind. The directly calculated total wave growth rate $r=0.919$ in this case. The corrected value determined by exponent $\alpha$ gives $r_{\text {exp }}=0.878$. In Fig. 21 we see qualitatively the same behavior as in Fig. 14 - very rapid tendency of solution to keep a limiting form: it takes less than $2 \mathrm{~h}$ to fit this form within a few percents near the spectral peak. At the solution periphery (both in frequency and in direction) the tendency to the limiting form is essentially weaker.

The comparison of Fig. 21 with its "academic" counterpart Fig. 14 illustrates the effect of source function - the only visible effect of "real" input as compared with "academic" one is in different evolution of non-self-similar background while the self-similar cores evolve in a universal way in both cases. Figures 22 and 23 illustrate the universality features of solutions quite similarly to Figs. 15 and 16. The "real" input solutions show the same universal shapes and the same scaling properties (bottom figures). Stress the remarkable closeness 

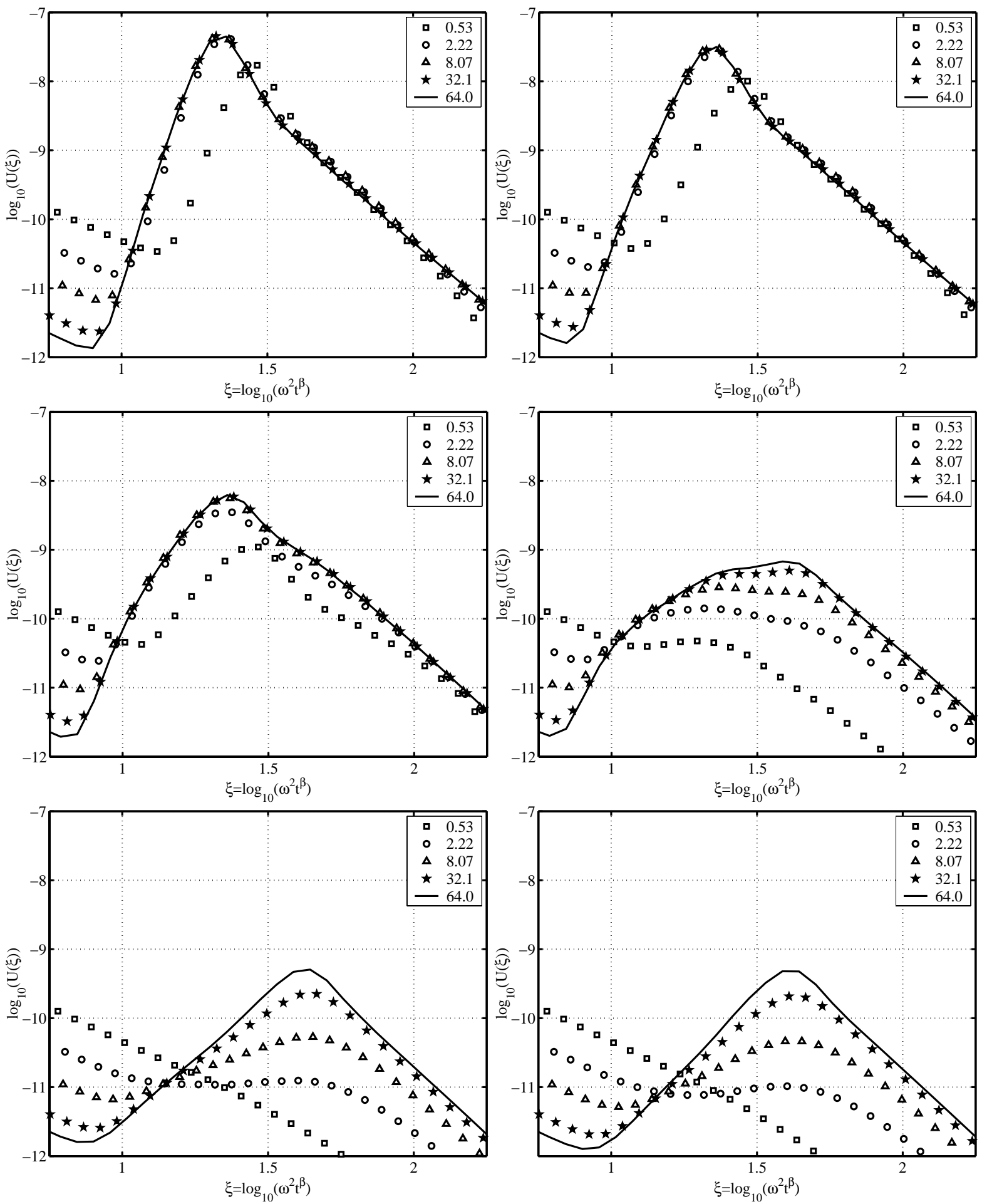

Fig. 21. Function $U(\xi)\left(\xi=|\boldsymbol{k}| t^{\beta}\right)$ for angles $0^{\circ}, 30^{\circ}, 60^{\circ}, 90^{\circ}, 150^{\circ}, 180^{\circ}$ for different times (see legend, in hours). Wave input is given by Eq. (51) for wind speed $10 \mathrm{~m} \cdot \mathrm{s}^{-1}$.

of fitting coefficients of the scaling in "academic" and "real" cases. There is nothing strange in this fact because the scaling parameters are determined by evident relation between parameters of self-similar solutions (Eqs. 94-96). They do not depend on details of source function but are determined by some integral characteristics of wind wave input only. The details of wind input, of course, are important at early stages of wave evolution and specific features of non-self-similar background are determined by these details but evolution of the self-similar core of solutions is identical in "academic" and "realistic" numerical experiments. 

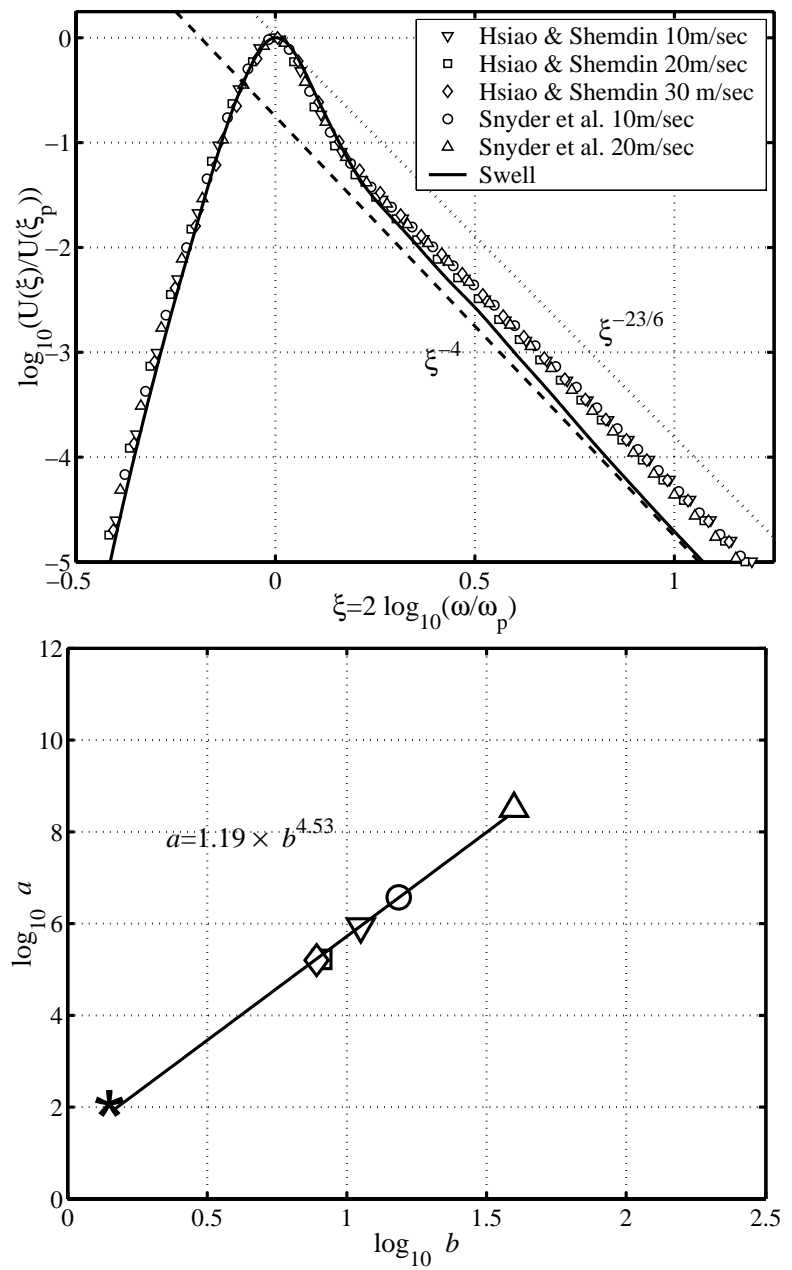

Fig. 22. Function $U(\xi) / U\left(\xi_{p}\right)$ as function of non-dimensional wave frequency for different wave inputs (in legend). The swell case is given by solid line. Bottom - the scaling of the solution peaks and the peak positions. Fitting formula and the corresponding fitting line are given.

A trivial but rather important result of all the above discussion is that wind speed $U_{h}$ in expressions for wave growth rates (Eqs. 47-52) is, evidently, not useful quantity for scaling wind-wave spectra. This characteristic can specify a freakish form of wave growth rates, while all our theoretical and numerical results show dependence on integral features of wave pumping, on fluxes.

\subsubsection{Solutions at large time - Evolution of mean values}

The evolution of mean wave field characteristics is a way to trace generic features of interplaying of nonlinearity and wave input. Figure 24 shows typical dependencies of basic wave parameters on time. In view of above remark on wind speed scaling we presented these dependencies in dimensional form for wave input by Hsiao and Shemdin (1983) and wind speed $10 \mathrm{~m} \cdot \mathrm{s}^{-1}$. The characteristics in Fig. 24 can be fitted by simple power-like dependencies for large times. The
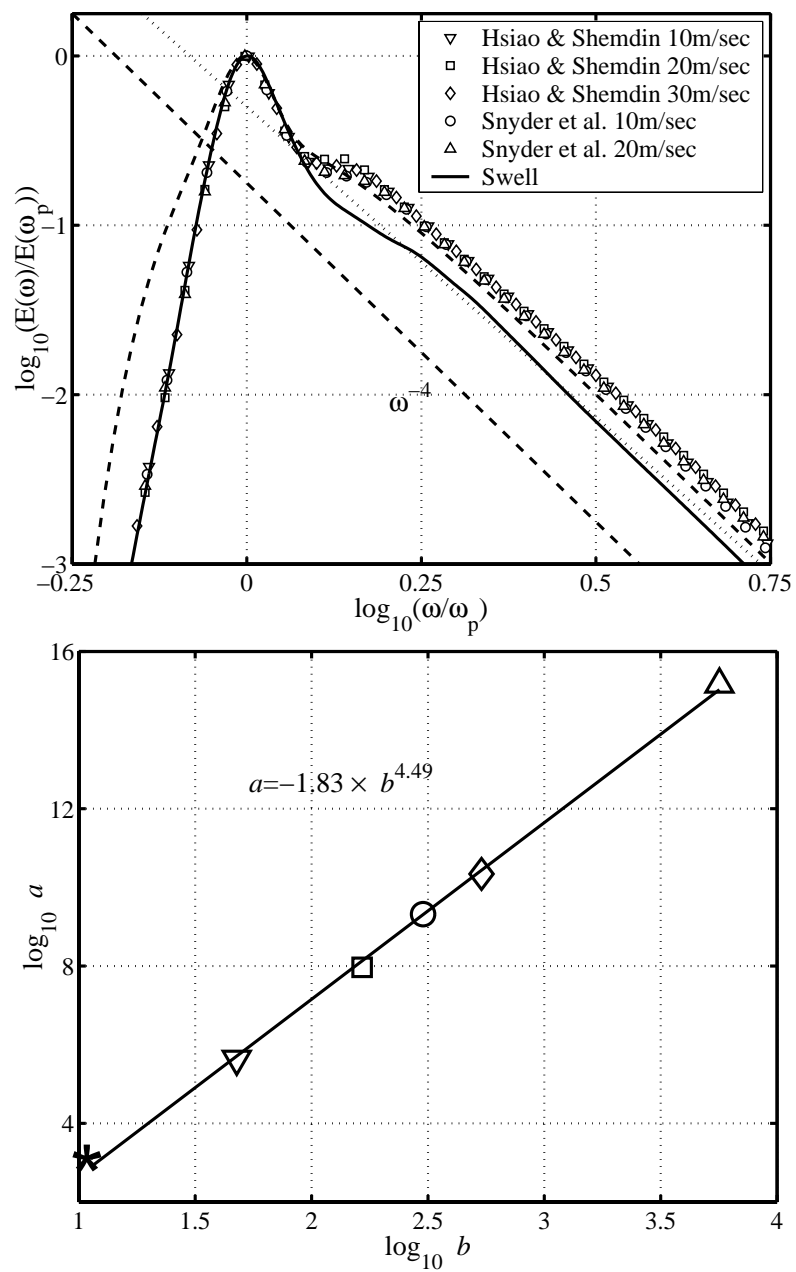

Fig. 23. Nondimensional frequency spectra $E(\omega) / E\left(\omega_{p}\right)$ as functions of non-dimensional wave frequency for different wave inputs (in legend). The JONSWAP spectrum for the standard peakedness $\gamma=3.3$ is shown by dashed curve. The dependence of the solutions parameters $a$ and $b$ is presented in bottom panel, swell scaling is given by $\star$.

corresponding exponents shown in legend have been calculated for times $t>4 \mathrm{~h}$ to remove effect of initial stage. Characteristic frequencies are traced as mean frequency $f_{m}$ and as a frequency of the solution peak $f_{p}$. One can see a small difference between two estimates of the corresponding exponents for these frequencies and for wave slopes.

This difference is illustrated by Table 6 for basic models of wave input and different wind speeds. The exponents $r, p, q$ were calculated for times more than $4 \mathrm{~h}$ in order to eliminate the contaminating effect of initial stage of evolution. Similar exponents (with indices "exp") were calculated from theoretical expression (Eq. 92) by power-like fit of peak values of numerical solutions (exponent $\alpha$ ). While exponents $p, r, q$ are responsible for global features of solutions, the exponents $p_{\text {exp }}, r_{\text {exp }}, q_{\text {exp }}$ can be associated with self-similar cores of numerical solutions. The corrections of exponents of energy $p$ and of characteristic frequency $q$ are of opposite signs, i.e. 

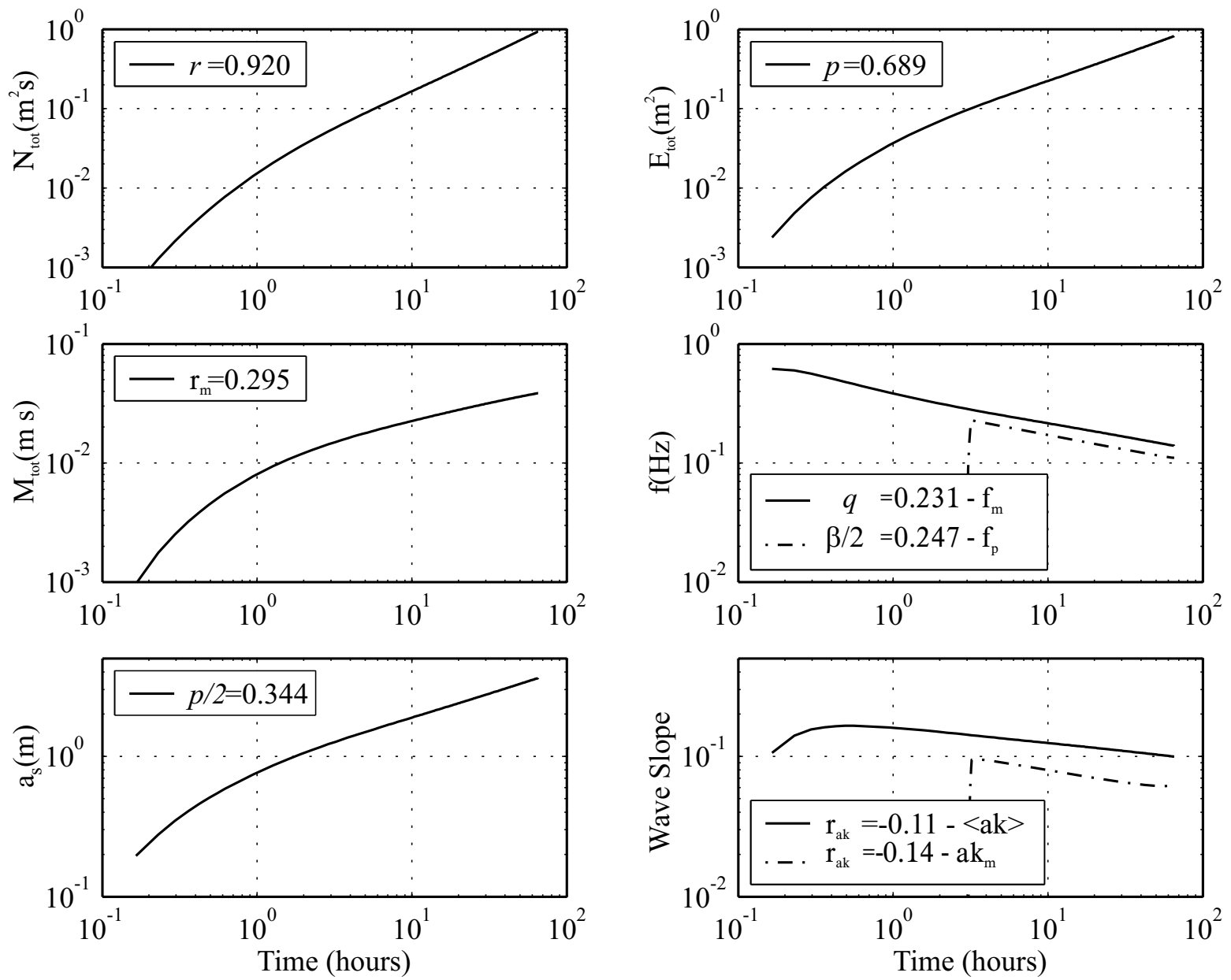

Fig. 24. Evolution of total wave action, energy, $x$-component of momentum, mean and peak frequency, significant wave height and wave slope (defined as $\langle a k\rangle$ and as $a k_{\text {peak }}$ ). Exponents of power-like approximations calculated for $t>4 \mathrm{~h}$ are shown in legends.

Table 6. Exponents of wind wave growth calculated for mean values $(r, p, q)$ and for peak characteristics $\left(r_{\exp }, p_{\text {exp }}, q_{\text {exp }}\right)$ of numerical solutions of the kinetic equation. Data for $t>4 \mathrm{~h}$ only are taken for the power-like approximations. Toba's exponents are given in the last column.

\begin{tabular}{|c|c|c|c|c|c|c|c|}
\hline Wave input & $\begin{array}{c}r \\
N(t)\end{array}$ & $\begin{array}{c}p \\
E(t)\end{array}$ & $\begin{array}{c}q \\
v(t)\end{array}$ & $\begin{array}{c}r_{\exp } \\
N\left(\omega_{p}\right)\end{array}$ & $\begin{array}{c}p_{\exp } \\
E\left(\omega_{p}\right)\end{array}$ & $\begin{array}{l}v_{\exp } \\
\omega_{p}\end{array}$ & $\begin{array}{c}T=\frac{p}{2 q} \\
\text { (Toba's exponent) }\end{array}$ \\
\hline \multicolumn{8}{|l|}{$10 \mathrm{~m} \cdot \mathrm{s}^{-1}$} \\
\hline Hsiao (1983) & 0.92 & 0.69 & 0.23 & 0.88 & 0.63 & 0.25 & 1.50 \\
\hline $\begin{array}{c}\text { Stewart (1974) } \\
\text { and Plant (1982) }\end{array}$ & 0.99 & 0.74 & 0.25 & 0.85 & 0.58 & 0.27 & 1.48 \\
\hline Donelan (1987) & 0.93 & 0.70 & 0.23 & 0.93 & 0.69 & 0.24 & 1.52 \\
\hline Snyder (1981) & 0.98 & 0.73 & 0.25 & 0.95 & 0.69 & 0.26 & 1.46 \\
\hline \multicolumn{8}{|l|}{$20 \mathrm{~m} \cdot \mathrm{s}^{-1}$} \\
\hline Hsiao (1983) & 0.94 & 0.70 & 0.24 & 0.91 & 0.66 & 0.25 & 1.46 \\
\hline Snyder (1981) & 1.05 & 0.79 & 0.26 & 0.98 & 0.70 & 0.28 & 1.52 \\
\hline \multicolumn{8}{|l|}{$30 \mathrm{~m} \cdot \mathrm{s}^{-1}$} \\
\hline Hsiao (1983) & 0.98 & 0.73 & 0.25 & 0.92 & 0.66 & 0.26 & 1.46 \\
\hline
\end{tabular}



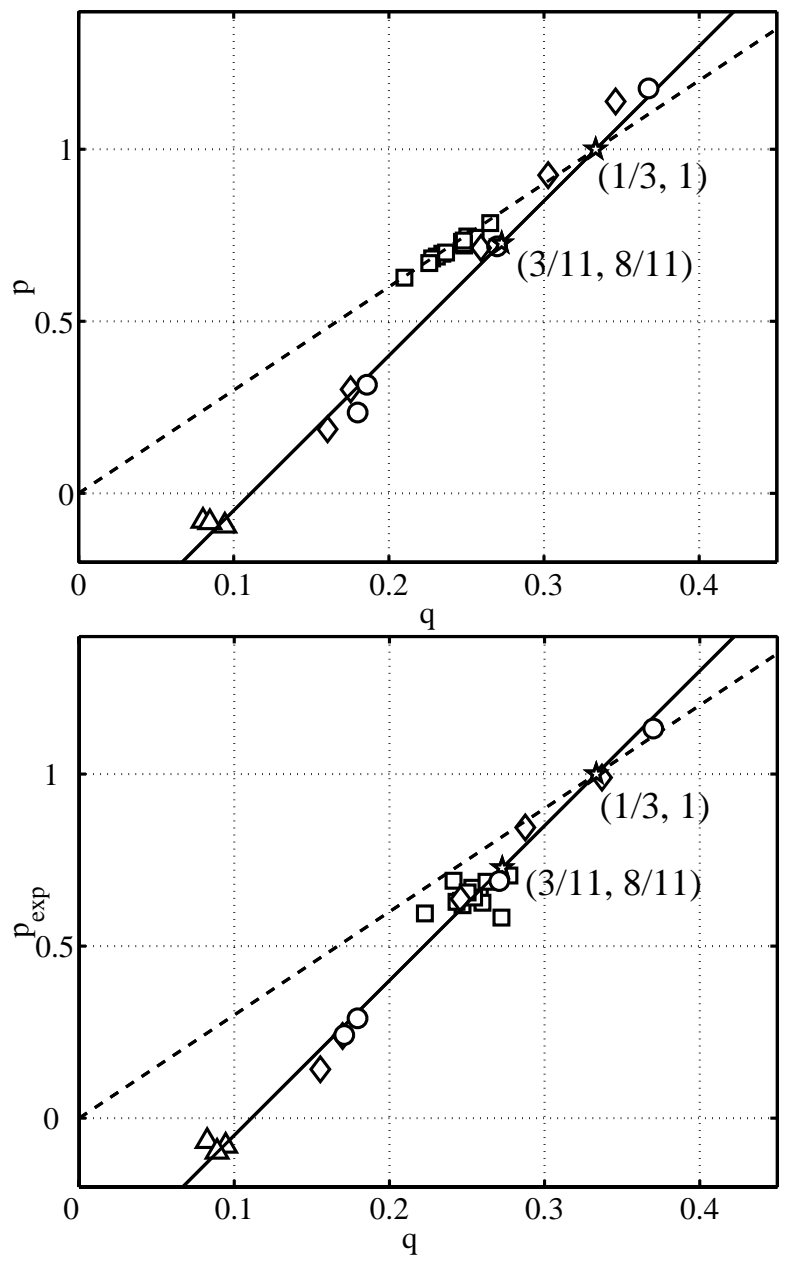

Fig. 25. Top - exponents $p$ and $q$ for power-like approximations of total energy and mean frequency of the kinetic equation solutions. Bottom - exponent $p_{\exp }$ and $q_{\exp }$ calculated for exponents of the solutions peaks $\alpha$ and $\beta$. $\bigcirc-$ Isotropic "academic" runs; $\diamond-$ Anisotropic "academic" runs; $\Delta-$ Swell; $\square-$ "Real" wave pumping. Exponents for constant wave action and wave energy inputs are given by stars. Hard line shows theoretical dependence of $p$ on $q$, dashed line corresponds to Toba's law.

wave energy and action of self-similar core grow slower than ones for the whole solution while characteristic frequency of the core falls faster. A possible explanation of the effect is that non-self-similar background is stronger for shorter waves where wave input is relatively strong and nonlinear transfer is weak due to low magnitudes of solutions.

Figures 25 gives a summary of directly calculated exponents $p$ and $q$ and their counterparts $p_{\text {exp }}$ and $q_{\text {exp }}$ derived from local features of the numerical solutions. Cases of swell, "academic" and "real" inputs are presented. One can see that all "academic" and swell cases follow perfectly the theoretical dependence $p(q)$ in a wide range of parameters of wave growth, i.e. the non-self-similar background affects the corresponding exponents rather slightly. In contrast, "realistic" runs have stronger dispersion. Corrected values (right panel) are fitted by theoretical dependence somewhat bet-
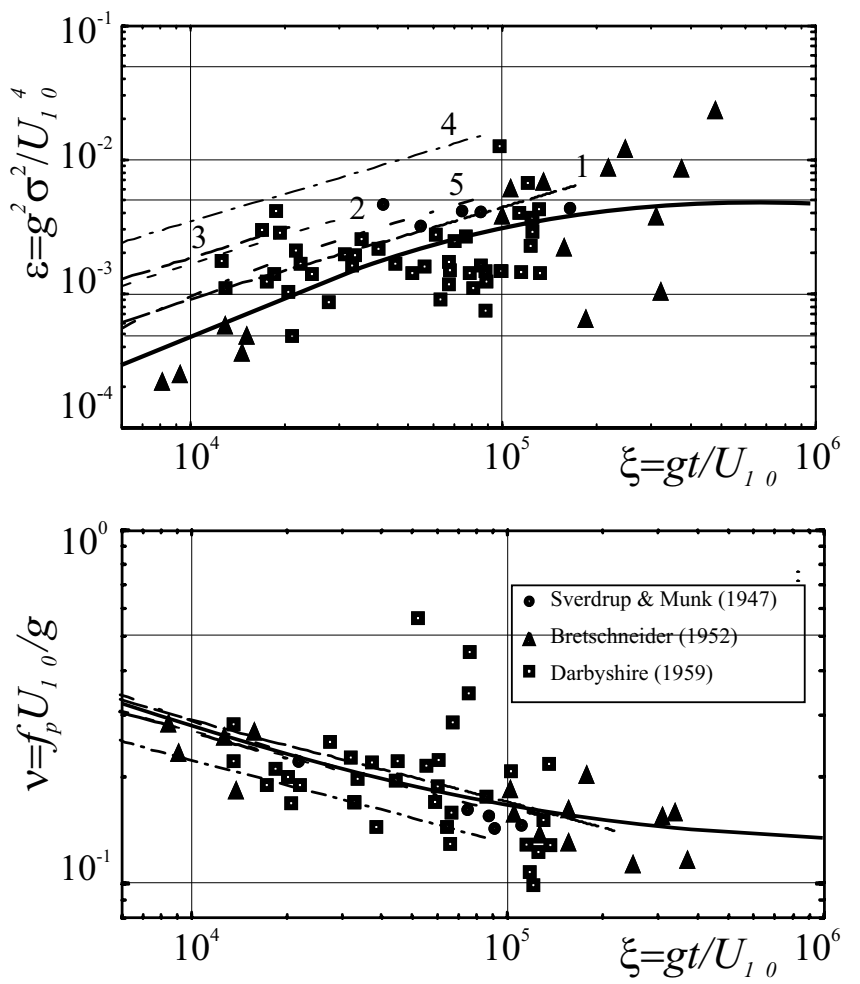

Fig. 26. Non-dimensional energy $\varepsilon=g^{2} \sigma^{2} / U_{10}^{4}$ (top) and frequency $v=f_{p} U_{10} / g$ (bottom) $v s$ non-dimensional duration $\xi=g t / U_{10}$. Numerical results for different wave input parameterizations are shown: wave input Hsiao and Shemdin (1983) curve $1-U_{10}=10 \mathrm{~m} \cdot \mathrm{s}^{-1}, 2-U_{10}=20 \mathrm{~m} \cdot \mathrm{s}^{-1}, 3-U_{10}=30 \mathrm{~m} \cdot \mathrm{s}^{-1}$; 4 - Donelan et al. (1987) with fixed $U_{\lambda / 2}=10 \mathrm{~m} \cdot \mathrm{s}^{-1} ; 5$ - Stewart (1974) and Plant (1982) wave input at $U_{10}=10 \mathrm{~m} \cdot \mathrm{s}^{-1}$. Recapitulative experimental data and their fit curve from Young's book (1999) are shown by symbols and hard line.

ter than by empirical Toba's law (Toba, 1973). The problem is that for "realistic" experiments these exponents are varying in very narrow range and difference between the experimental Toba law and the theoretical dependence cannot be demonstrated in full measure.

Figure 26 can be considered as an illustration of reasonable agreement of our numerical results with experimental data discussed in monograph by Young (1999). The dispersion of our numerical results is of the same order as dispersion of experimental points. A possible source of the dispersion is in normalization of results. Normalization of mean energy as $U^{-4}$ (wind speed in power minus four!) requires rather high accuracy of measurements of wind speed. Moreover, as we concluded above, not the wind speed itself but some integral properties of wind input are responsible for wave growth. An additional reason for mismatch is the use of explicit dependence on time $t$. Duration of initial (non-selfsimilar) stage depends essentially on initial state that can lead to incorrect comparison of results.

Figure 25 and Table 6 show clearly that local features of solutions (position of the solution peak and this peak magnitude) are likely more adequate for comparison of results. 

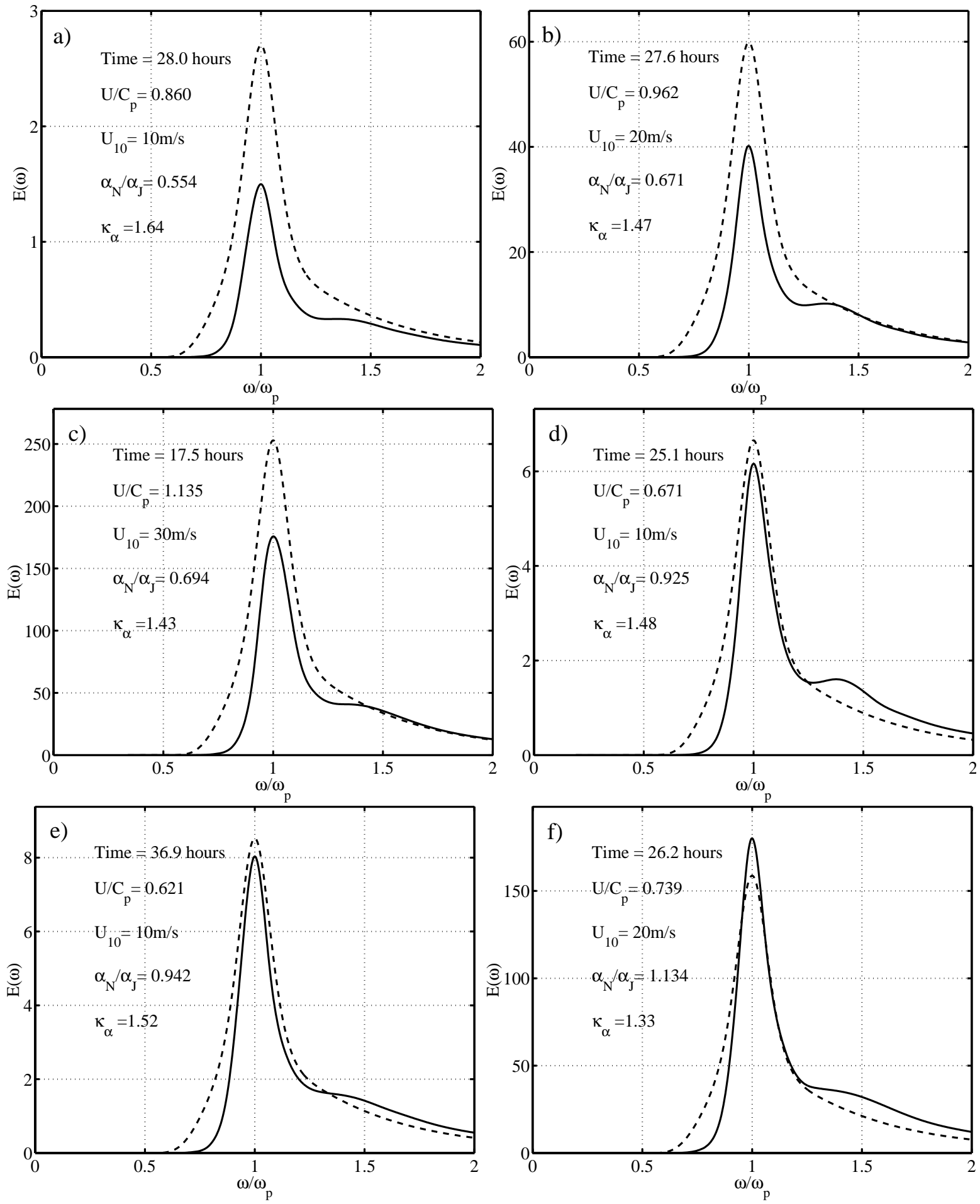

Fig. 27. Comparison of JONSWAP (dashed) and numerical (hard line) spectra for different wave input. (a), (b), (c) wave input by Hsiao and Shemdin (Eq. 51) for wind speeds $10,20,30 \mathrm{~m} \cdot \mathrm{s}^{-1}$ correspondingly; (d) wave input by Donelan and Pierson-jr. (Eq. 52) for $U=10 \mathrm{~m} \cdot \mathrm{s}^{-1}$; (e), (f) wave input by Snyder et al. (Eq. 47). Time in hours, inverse wave age $U / C_{p}$ and wind speed $U$ are shown in plots. Exponent $\kappa_{\alpha}$ (Eq. 39) was estimated by power-like dependence of the solution peak on time. Solutions for $t>4 \mathrm{~h}$ was taken to reduce the effect of initial stage of wave growth. Ratio of JONSWAP and numerical spectra peak magnitudes is given as $\alpha_{N} / \alpha_{J}$.

\subsection{Numerical solutions vs. JONSWAP spectrum}

Basing on theoretical results and on observations made in the above subsections one can perform a comparison of nu- merical and JONSWAP spectra for the same values of wave ages. Results of the comparison are shown in Fig. 27. Numerical spectra (frequency spectra integrated in angle) were calculated for different parameterizations of wind input and 

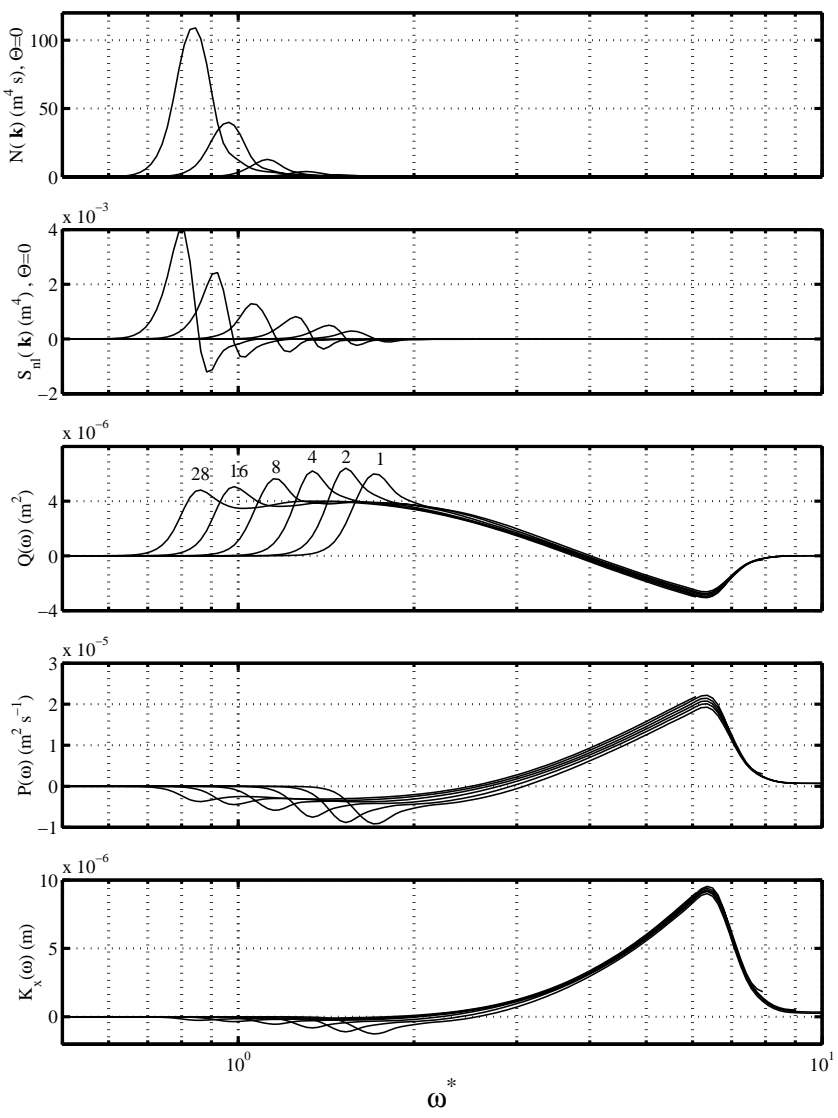

Fig. 28. Down-wind spectra of wave action, nonlinear transfer term $S_{n l}$ and fluxes of wave action $Q(\omega)$, wave energy $E(\omega)$ and wave momentum $K_{x}(\omega)$ as functions of nonlinear frequency $\tilde{\omega}=\omega U_{10} / g$ at different time (in hours in the center panel). Wave input as in Hsiao and Shemdin (1983), wind speed $10 \mathrm{~m} \cdot \mathrm{s}^{-1}$.

wind speeds presented in Table 6. For the JONSWAP spectra peakedness parameters were fixed $(\gamma, \sigma$, in Eq. 38) while the exponents of wave growth $\kappa_{\alpha}$ (Eq. 39) have been calculated in accordance with (Eq. 110), i.e. for exponents that characterize evolution of self-similar core of solutions. The parameter $\alpha_{0}=0.08 /(2 \pi)$ was fixed (see Eq.40 in Babanin and Soloviev, 1998)). Thus, we fix ratio of peak magnitudes of numerical and JONSWAP spectra for large times when numerical solutions keep self-similar forms. The ratio shown in plots as $\alpha_{N} / \alpha_{J}$ varies in a wide range. At the same time it is well within dispersion of parameter $\alpha_{J}$ found in sea measurements (Babanin and Soloviev, 1998, Table 2). The ratio is lower for wave input by Hsiao and Shemdin (1983) (Fig. 27a, b, c) and higher for generation rates by Donelan and Piersonjr. (1987) (Fig. 27d) and Snyder et al. (1981) (Fig. 27e, f). This is in perfect agreement with our comparison of different generation rates in Sect. 3.4.1. Besides that $\alpha_{N} / \alpha_{J}$ depends on wind speed for the same input parameterization (compare Figs. 27a, b, c for wave input by Hsiao and Shemdin, 1983, or Fig. 27e, f for input by Snyder et al., 1981). This is an additional illustration of trivial remark made above: not the wind speed $U_{10}$ itself but the integral value of input term $S_{i n}$ is responsible for scaling of wind-wave spectra.

Figure 27 demonstrates rather good conformance of numerical and JONSWAP spectral shapes (compare with "academic" Figs. 16 and 23). At the same time, shapes of frequency spectra $E(\omega)$ are not universal for numerical spectra - plateaus or, sometimes, peaks are seen in the spectra at frequencies approximately $40 \%$ higher than the peak ones (e.g. Fig. 27b, d). This deviation from universality looks quite natural for the frequency spectra that can cumulate both selfsimilar core and non-self-similar wave background in the same frequency range.

\section{Self-similarity of nonlinear transfer}

\subsection{Asymptotics of collision integral and of spectral fluxes}

The discussion of self-similarity features of nonlinear transfer is extremely important to finalize our study. The term $S_{n l}$ itself and the resulting fluxes of constants of motion determine predominantly the evolution of wind-wave spectra. The typical evolution of all the terms is presented in Fig. 28. In the whole frequency range $(0.02-2 \mathrm{~Hz})$ we see a combination of fluxes of different signs. Near the spectral peak the behaviour of fluxes agrees with the above theoretical scheme: all fluxes (action, energy and momentum) are directed to large scales. The domain of inverse cascades is expanding with time to low frequencies while the boundary of direct cascades is tending to some limit. These direct cascades of wave action, energy and momentum are maintained by strong dissipation that arrests all the fluxes in high frequencies (at $\tilde{\omega}>6.5$ ).

The time dependence of the terms for fixed nondimensional frequencies is shown in Fig. 29. Four values of $\tilde{\omega}$ are taken: $0.9,1.0,1.2,1.5$. The particular case of wave input corresponds approximately to the case of slowly decaying wave action input with $r=0.92, r_{\text {exp }}=0.88$ (see Table 6). Power-like theoretical dependencies are shown in bold for the constant wave action input $(r=1)$. One can see rather good agreement with theoretical predictions.

The self-similar form of the kinetic Eq. (98) allows to detail the relevance of our numerical experiments to theoretical results. After re-scaling the collision integral takes a form

$$
\lim _{t \rightarrow \infty} S_{n l}(\boldsymbol{k}, t)=\left[\alpha N(\boldsymbol{k})+\beta \boldsymbol{k} \nabla_{\boldsymbol{k}} N(\boldsymbol{k})\right] / t
$$

Thus, the asymptotics of nonlinear transfer quantities $S_{n l}, Q$, $P, K_{x}$ can be calculated from instantaneous solutions and than be compared with direct calculations of $S_{n l}$ and fluxes. Such comparison can be considered both as theory verification and as a test of numerical approach.

Results of the comparison are presented in Fig. 30 for swell solutions. As it was mentioned above the transition to "inherent" wave spectra occurs for rather short time. For the swell case considered above this time is about half an hour (see Sect. 5.2.1 and Fig. 9). Patterns for $0.5 \mathrm{~h}$ and $100 \mathrm{~h}$ of swell evolution are almost identical. Small differences 

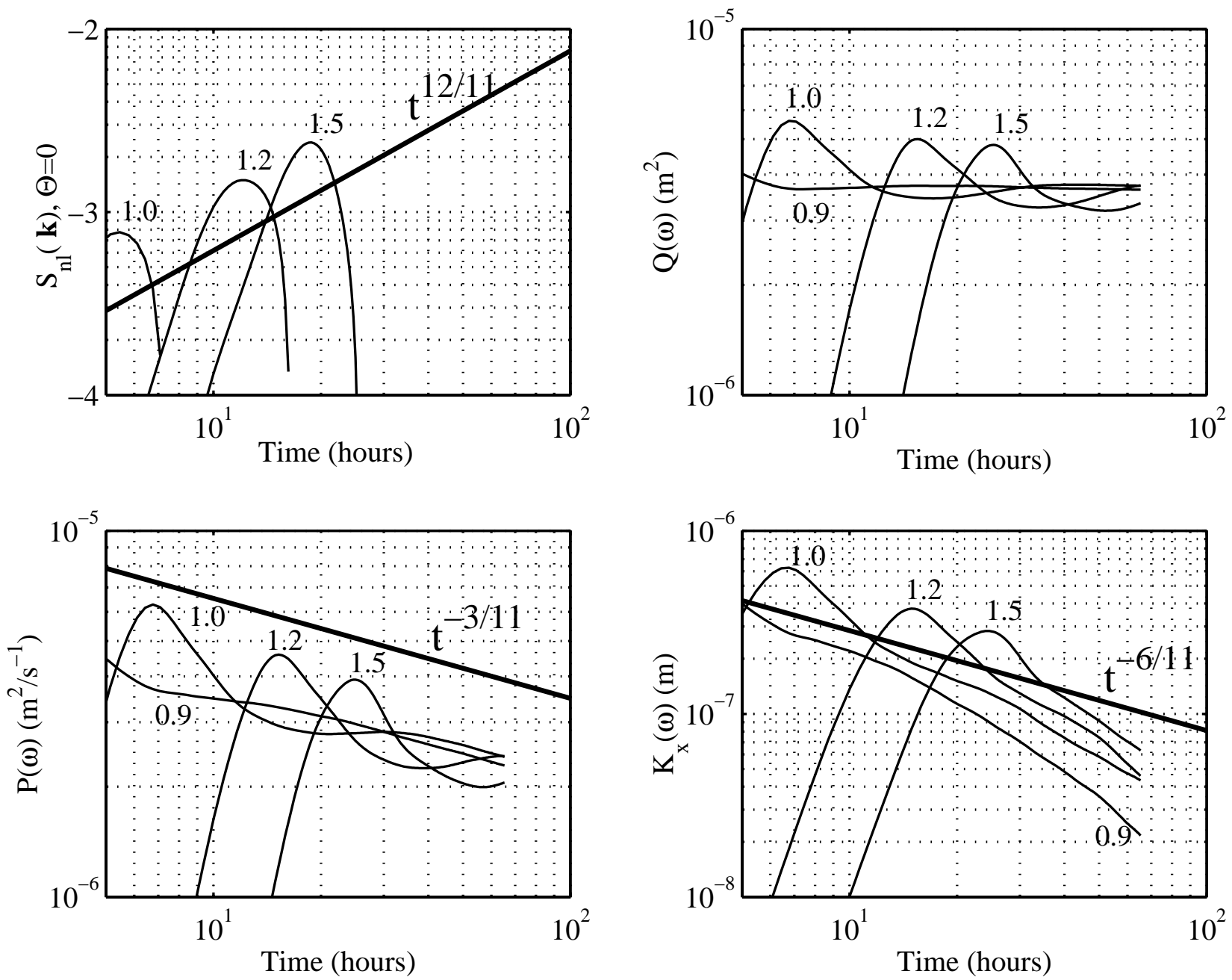

Fig. 29. Nonlinear transfer term $S_{n l}$ (a), wave action flux $Q$ (b), energy flux $P$ (c) and momentum flux $K_{x}^{*}$ (d) as functions of time for different non-dimensional frequencies $\tilde{\omega}=\omega U_{10} / g: \tilde{\omega}=0.9,1.0,1.2,1.5$ (shown as curve markers). Wind speed $U_{10}=10 \mathrm{~m} \cdot \mathrm{s}^{-1}$, wind input (Eq. 51). Power-like asymptotics for constant wave action input are shown (see Table 3).

appear near peaks of distributions. The hybrid nature of weakly nonlinear cascading in the swell case is clearly seen in these patterns - energy flux changes its sign at $\kappa / \kappa_{p} \approx 2$ $\left(\omega / \omega_{p} \approx 1.4\right)$. For momentum flux the point of the cascade inversion is closer to the spectral peak at $\kappa / \kappa_{p} \approx 1.5$.

For the case of wave input the tendency to an asymptotic behaviour is slower but it is still pronounced. Figure 31 illustrates it quite well. The nonlinear transfer term $S_{n l}$ for down-wind direction is shown to be very close to its asymptotic form while for fluxes, which are averaged in angle values, the tendency is essentially slower. The explanation is rather simple. We see again the effect of averaging in angle when non-self-similar background contaminates the strong features of self-similarity.
7.2 Self-similar solutions and the Kolmogorov-Zakharov cascades

In our analysis of self-similar solutions in Sect. 5.2 we noted the generic relationship of self-similar non-stationary (nonhomogeneous) solutions and the Kolmogorov-Zakharov stationary solutions of the kinetic equation: formally, the $\mathrm{KZ}$ solutions are exact solutions of the kinetic equation in selfsimilar variables: the term corresponding to non-stationarity (non-homogeneity) and nonlinear transfer term vanish independently for these special solutions. We concluded quite naturally, that the $K Z$ solutions can be treated as stationary limits of non-stationary (non-homogeneous) self-similar solutions for the two particular cases - constant wave action input and constant wave energy input. There is nothing behind the above statement but behaviour of slopes of spectral tails of these solutions. In fact, in addition to the fundamental exponents there are fundamental coefficients - the so-called Kolmogorov's constants that are responsible for ra- 

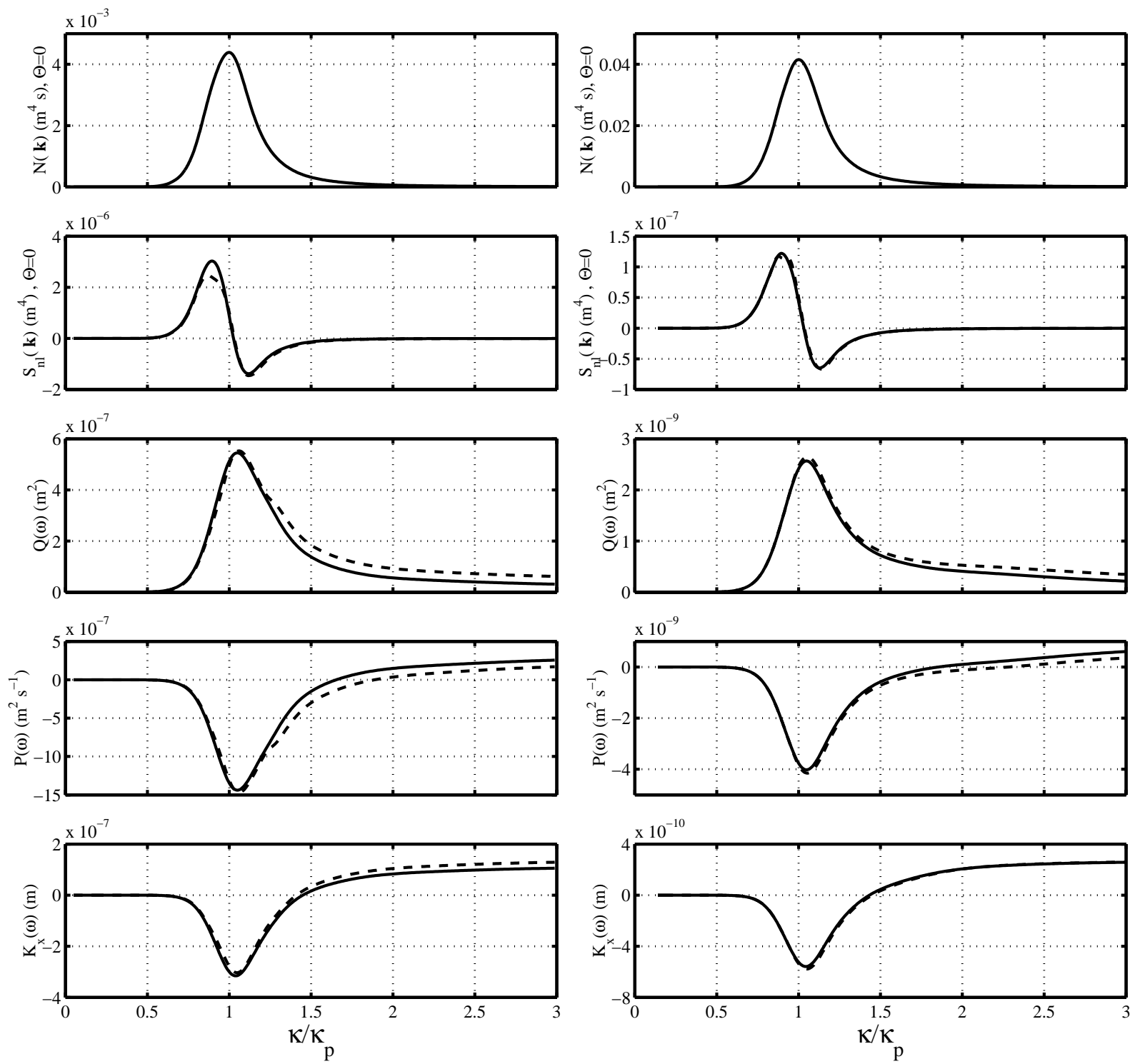

Fig. 30. Direct calculation of $S_{n l}$ and fluxes for swell (solid lines) vs. their asymptotical profiles predicted by Eq. (98) (dashed). Left panel - time $0.5 \mathrm{~h}$; right - time $100 \mathrm{~h}$.

tio of spectra magnitudes to spectral fluxes (in power 1/3). These constants can be estimated analytically (Geogjaev and Zakharov, 2005) ${ }^{1}$ for the KZ solutions. A number of questions arises when we try to estimate the Kolmogorov constants numerically.

The Kolmogorov constants $C_{p}, C_{q}$ are introduced by Eqs. (74 and 75) for isotropic stationary solutions. The numerical solutions are essentially non-stationary and, more, anisotropic. The effect of anisotropy is eliminated automatically in definitions themselves where frequency spectral distributions are used (Eqs. 73 and 77). More burning questions are: How to define the Kolmogorov constants for nonstationary (non-homogeneous) case? Does the definitions make a physical sense? How the corresponding values can be related to "true" Kolmogorov constants?.
Probably, these constants can be estimated for quasistationary parts of solutions - their high-frequency tails. It was made numerically for the direct cascade constant $C_{p}$ (Pushkarev et al., 2003; Lavrenov et al., 2002) within the specially designed "academic" runs. Isotropic (or anisotropic) wave generation was put at the low frequency end of the calculation domain while strong dissipation was set up at the high frequency end. The wide inertial frequency domain provided a quasi-constant spectral flux of energy from large to small scales. In this case the solution reaches a quasistationary state in an explosive way and leaves no questions on the first Kolmogorov constant $C_{p}$ : the numerical estimate tends quite rapidly to some limiting value.

The situation is quite different in case of our key interest - self-similar inverse cascade. The corresponding nonstationary solutions propagate in front-like manner with fi- 

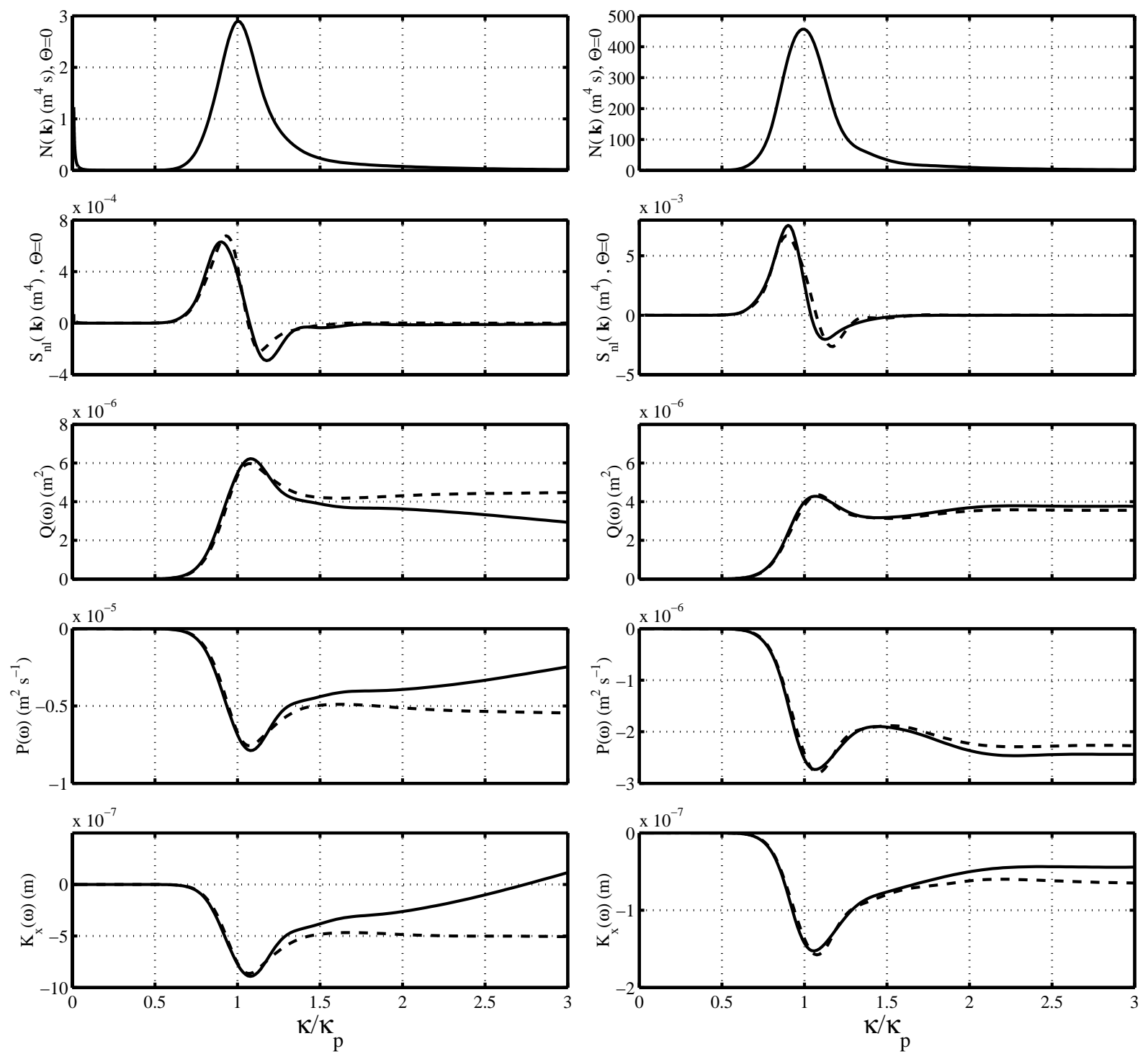

Fig. 31. Direct calculation of $S_{n l}$ and fluxes for swell (solid lines) vs. their asymptotical profiles predicted by Eq. (98) (dashed) for wave input (Eq. 51), wind speed $10 \mathrm{~m} \cdot \mathrm{s}^{-1}$. Left panel - time $4.15 \mathrm{~h}$; right - time $65 \mathrm{~h}$.

nite speeds (see Fig. 31). Moreover, for the family of self-similar solutions with different exponents of wind wave growth the wave action flux depend on time essentially. The prospects for building a bridge from the $\mathrm{KZ}$ solutions to the families of non-stationary and non-homogeneous self-similar solutions look illusory.

A straightforward trick to overcome the difficulty is to assume the value $C_{q}$ be slowly dependent on time (fetch). In fact, the correct solution of the problem does not require this trick: The ratios of the self-similar solutions to fluxes for the self-similar solutions defined as the KZ constants $C_{p}$ and $C_{q}$ do not depend on time!

Consider, first, numerical evidence of the result. In Fig. 32 the calculation of the Kolmogorov constant $C_{q}$ for two cases of wave input is presented. Wave inputs (Eqs. 51 and 47) for $U_{10}=10 \mathrm{~m} \cdot \mathrm{s}^{-1}$ are taken for comparison. Despite great quantitative difference of the examples (wave action by factor 3, flux $Q-5-7$ times) the resulting estimate for the Kol- mogorov constant $C_{q}$ shows evident tendency to an asymptotic value $C_{q} \approx 0.35$. The range where this value depends slightly on wave scales (constant flux domain) correspond to non-dimensional wavenumbers $\kappa / \kappa_{p} \approx 2-4$ (1.5-2 in nondimensional frequencies).

Even more cogent argument for the constant $C_{q}$ is given in Fig. 33. Two "academic" runs with essentially different wave growth rates $r=1 / 2$ (wave action input decays) and $r=4 / 3$ (growing wave action, constant wave energy input) are presented. Variations of wave action flux reach 4 times in these two examples but the values $C_{q}$ remain very close to the previous estimates.

The dependence of $C_{q}$ on time (fetch) can be found easily for self-similar solutions using scaling relations given in Tables 2-5.

$$
C_{q}(\omega, t)=\lim _{t \rightarrow 0} \frac{E(\omega, t) \omega^{11 / 3}}{g^{4 / 3} Q(\omega, t)^{1 / 3}}=C_{q}(|\xi|)
$$



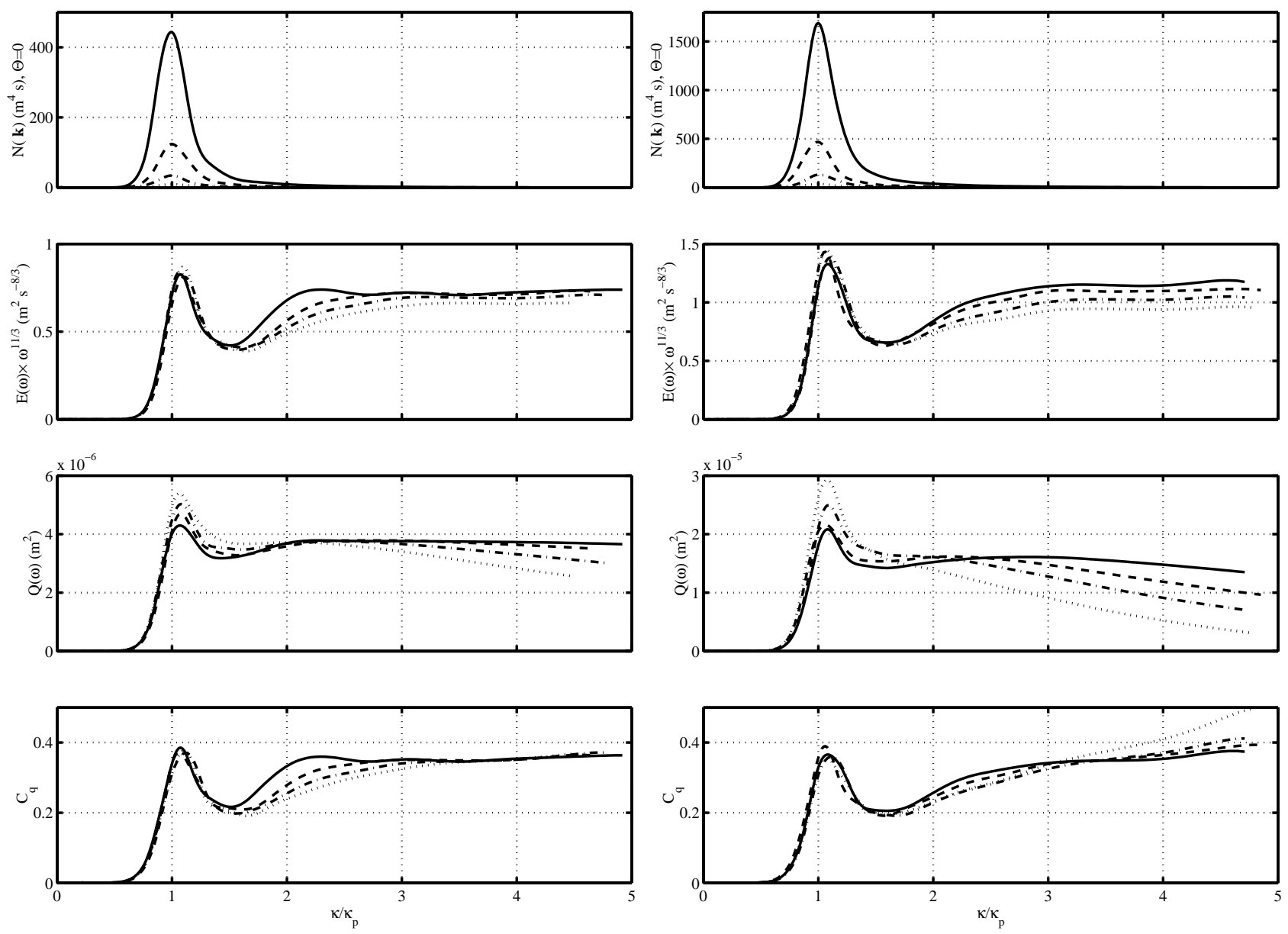

Fig. 32. Down-wind solution $N(\boldsymbol{k})$, compensated frequency spectra of energy $E(\omega) \omega^{11 / 3}$, wave action flux $Q$ and the resulting estimate of the Kolmogorov constant $C_{q}$. Left - wave input (Eq. 51), wind speed $10 \mathrm{~m} \cdot \mathrm{s}^{-1}$, time 8 (dotted), 16 (dash-dot), 32 (dashed), $64 \mathrm{~h}$ (hard line); right - wave input (Eq. 47), wind speed $10 \mathrm{~m} \cdot \mathrm{s}^{-1}$, time 4 (dotted), 8 (dash-dot), 16 (dashed), $32 \mathrm{~h}$ (hard line).

Thus, the analogue of the inverse cascade Kolmogorov constant $C_{q}$ does not depend on time, it depends on self-similar argument only. Quite similarly, for the direct cascade constant $C_{p}$ one has

$$
C_{p}=\lim _{t \rightarrow 0} \frac{E(\omega, t) \omega^{4}}{g^{4 / 3} P(\omega, t)^{1 / 3}}=C_{p}(|\xi|)
$$

Thus, the basic property of the KZ solutions - constant ratio of the solution to the fluxes (in power $1 / 3$ ) is dealing with self-similarity of solutions of the Hasselmann equation. It remains valid in a general case irrespectively to the case of wave pumping. This extends essentially the applicability of the concept of Kolmogorov's cascading for non-stationary (non-homogeneous) evolution of wind-wave spectra.

Figure 34 (left) illustrates this conclusion for an artificial "hybrid" solution of coexisting direct and inverse cascades presented above (Fig. 7). In ranges of weak variability of fluxes the corresponding functions of self-similar argument $C_{q}(\xi), C_{p}(\xi)$ tend to some limiting values. Inverse cascade of wave action in a range $\kappa / \kappa_{p} \approx 2-5\left(\omega / \omega_{p} \approx 1.5-2.2\right)$ is governed by slowly varying parameter $C_{q} \approx 0.35$. In a large high-frequency domain the direct cascade is described perfectly well by the $\mathrm{KZ}$ solution with $C_{p} \approx 0.38-0.4$. The domains of inverse and direct cascades in this case are determined by wave input features. These domains coexist in the swell case as it was pointed out above but the inverse cascade domain for energy is rather narrow $\left(\kappa / \kappa_{p}<2\right)$. Figure 34 (right) illustrates this special "hybrid" cascading. The ratios (Eqs. 112 and 113) give values which are very close to numerical and theoretical estimates of the "true" Kolmogorov constants found previously (Lavrenov et al., 2002; Geogjaev and Zakharov, 2005 ${ }^{1}$; Pushkarev et al., 2003).

\section{Discussion}

In this paper we have attempted to address important questions of wind-driven eave dynamics based on "first principles" of the statistical description of waves. We recognize that to some researchers these principles may appear too restrictive for current needs. On one hand, hypotheses, approximations, and simplifications are unavoidable. On the other 

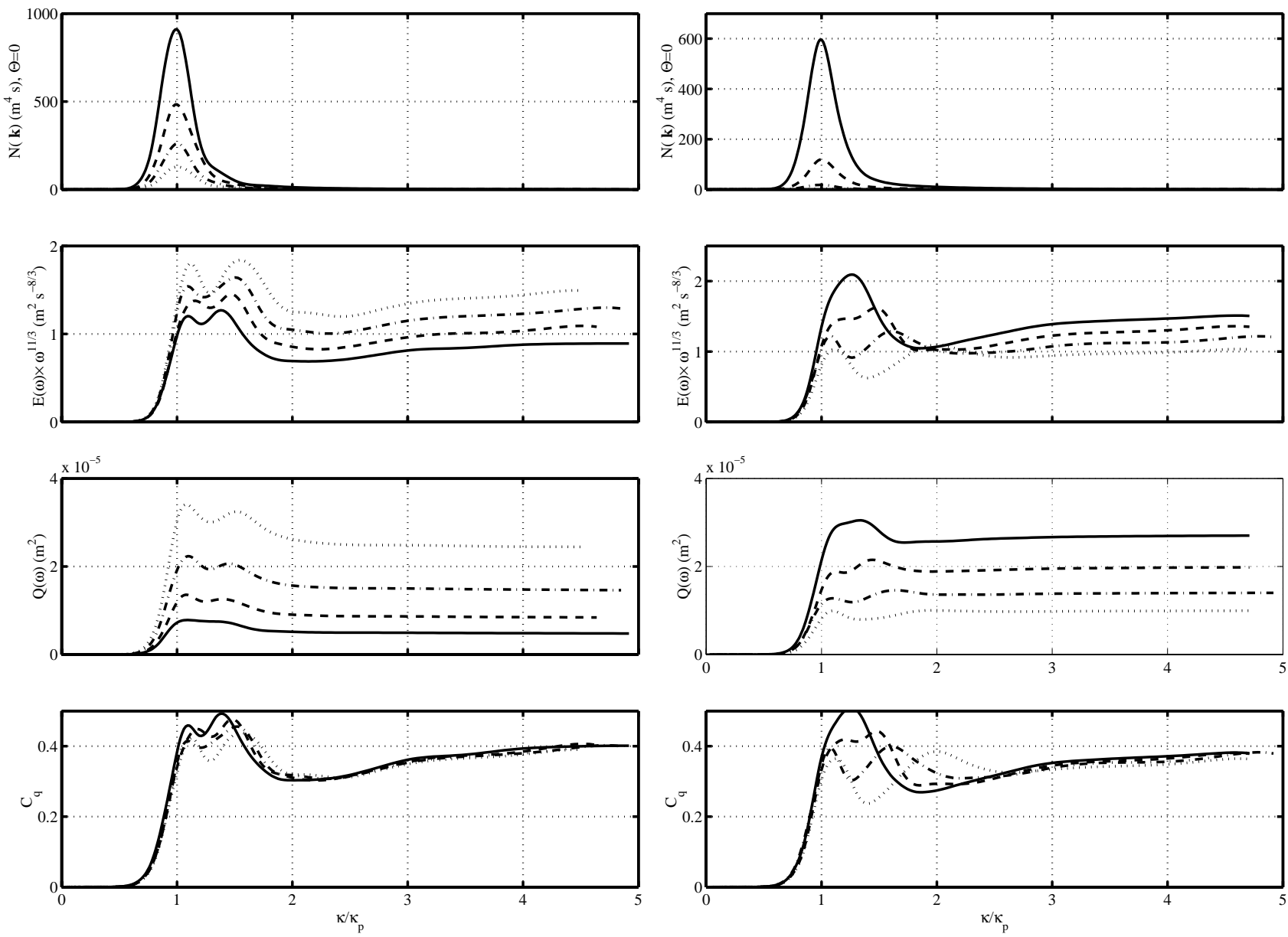

Fig. 33. Down-wind solution $N(\boldsymbol{k})$, compensated frequency spectra of energy $E(\omega) \omega^{11 / 3}$, wave action flux $Q$ and the resulting estimate of the Kolmogorov constant $C_{q}$. Left - "academic" wave input $N_{t o t} \sim t^{1 / 2}$ time 4 (dotted), 8 (dash-dot), 16 (dashed), $32 \mathrm{~h}$ (hard line); right "academic" wave input $N_{t o t} \sim t^{4 / 3}$, time 4 (dotted), 8 (dash-dot), 16 (dashed), $32 \mathrm{~h}$ (hard line).

hand, we argue here that first principles can provide an excellent framework for properly considering these hypotheses, approximations and simplifications.

We started with formulation of the basic principles in Sect. 2 of the paper. In Sect. 3 we fixed common points and points of disagreement in experimental and theoretical approaches. The experimental parameterizations of wind-wave spectra are based on the concept of self-similar evolution of wave field, they are implying independence of the resulting spectra on details of wind-wave generation and dissipation. At the same time, a great portion of efforts in parameterizations of wind-wave growth and dissipation is aimed at detailing frequency dependences of the rates. It should be realized quite clearly, that any "new" form of these dependencies implies an extra scaling. Physical reasons for the extra scaling are usually absent in the models. Great dispersion of magnitudes of the rates is just one side of the problem. Other side of the problem, mentioned in Sect. 3: the forms of input functions (e.g. their low-frequency cutoff) are not consistent with self-similar scaling of wind-wave spectra.
Often in numerical models of wind waves known physical principles are sacrificed for speed of computations, algorithm stability, and "plausibility" of the results. Evidently, it is easier to achieve the plausibility by tuning generation/dissipation terms in the kinetic equation. The resources of such "diagnostic" patching (Tolman and Chalikov, 1996) of the physical problem are rather short and, additionally, the patching being applied for particular conditions does not guarantee adequate results in general case. We propose our "first principles" - the theory of weak turbulence - as a physical basis, as a system of criteria for verification of wind-wave models but not as a barrier for experiments with different models.

The theory of weak turbulence predicts correctly many features of wind-wave evolution, first of all, the well-known power-like asymptotics of wind wave spectra. This fact is usually ignored because the corresponding $\mathrm{KZ}$ solutions are considered as physically irrelevant - these solutions are isotropic and are not localized in wave scales. But the KZ solutions are not "the first and the last" result of the theory 

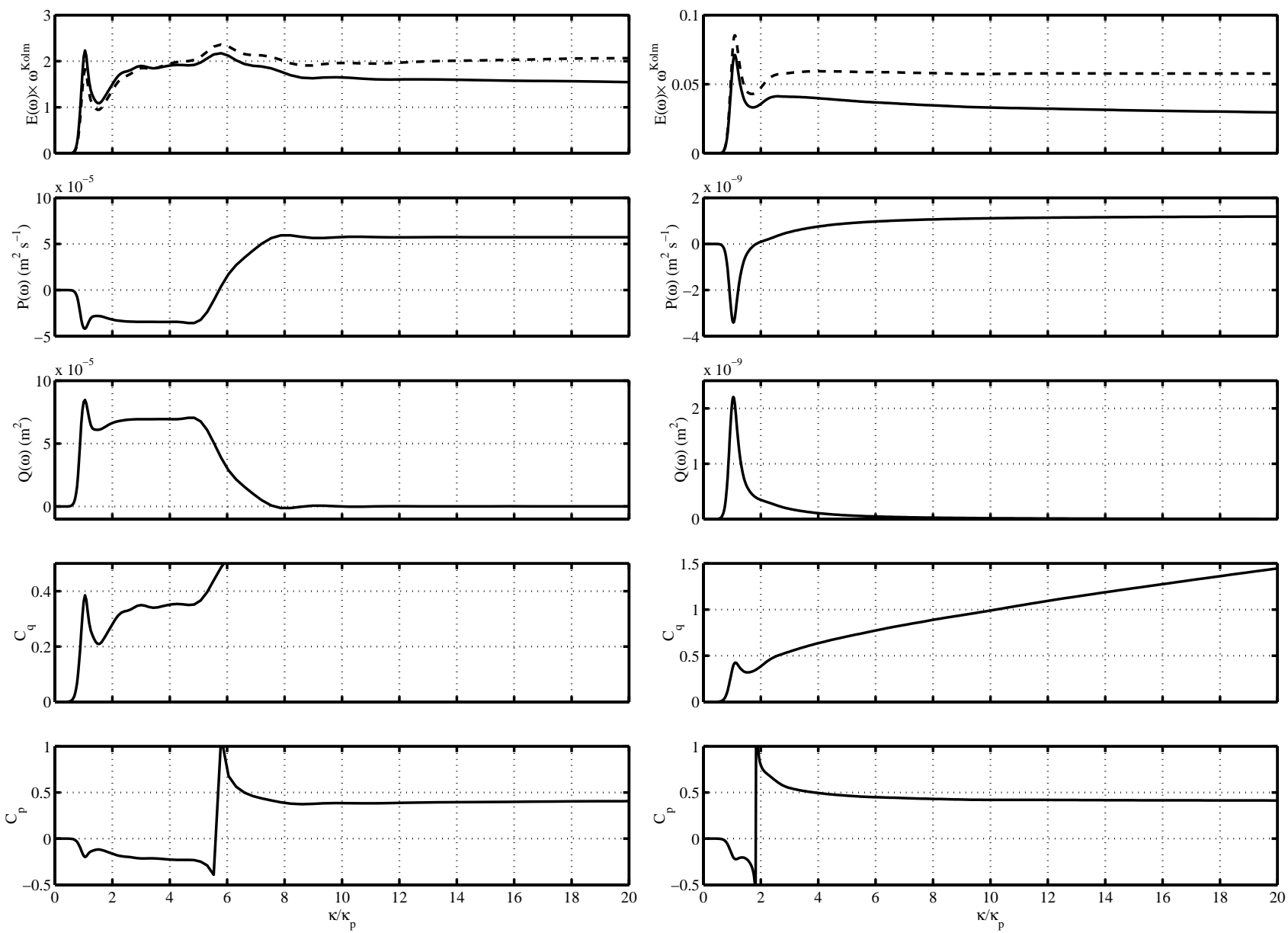

Fig. 34. Correlation of wave spectra and spectral fluxes. Compensated spectra for direct (dashed) and inverse (hard line) cascades, wave energy flux, wave action flux and estimates for the Kolmogorov constants $C_{q}$ and $C_{p}$ (Eqs. 113, 112). Left column - "artificial" wave input (see Fig. 7), both direct and inverse cascade regimes are presented by wide domains of quasi-constant fluxes. Right column - swell case, direct cascade with quasi-constant (in frequency) flux of wave energy allows for reliable estimate of the Kolmogorov constant $C_{p}$. The domain of inverse cascade is relatively narrow.

of weak turbulence. In fact, they represent basic physical mechanism of wind-wave evolution - cascading of wave action, energy and momentum in wave scales. Evidently, this mechanism works in general case and, more, it dominates in many cases of interest.

The theoretical analysis in Sect. 5 assumes that wave nonlinearity is a dominant process in wind-wave generation. As the first step of the approximation procedure we consider solutions of "conservative" Hasselmann Eq. (4) for deep water waves to describe shapes of self-similar solutions. The next step - the balance Eq. (5) just selects a self-similarity index that is consistent with the growth of total wave action (energy). We show that the families of self-similar solutions for duration-limited and fetch-limited wave growth are nothing but generalization of the KZ solutions where spectral fluxes are not yet constant but power-like functions of time (fetch). These solutions describe inverse cascades of wave action, energy and momentum for "acceptable" rates of wave growth. Particular case of swell corresponds to "hybrid" regime when wave action propagates to low frequencies while inverse cascade of wave energy and momentum fluxes occur in a narrow band near spectral peak only. The direct cascades of wave energy and momentum play a key role in wave evolution: they provide leakage of wave energy and momentum in high frequencies without any dissipation in the system.

The self-similarity of experimental parameterizations of wind-wave spectra (e.g. JONSWAP) makes possible to relate the indexes of self-similar solutions with experimental exponents of wind-wave growth. The experimental dependencies are shown to be consistent with the theoretical results. This is an important point for further numerical analysis.

Numerical approach of Sect. 6 was described in previous papers (see Tracy and Resio, 1982; Resio and Perrie, 1991; Pushkarev et al., 2003) and is presented in the paper briefly. The point of the present numerical study is in formulation of physical models basing on the concept of self-similarity. The "academic" series allows one to obtain the self-similar behaviour in "naked form" in a whole range of indexes of 
self-similarity. The main result of the section and of the paper as a whole: Shapes of spectra depend very slightly on the indexes. This result can be considered as a theoretical argument for universal parameterization of shapes of wind-wave spectra. In fact, the universality of the spectral forms is a starting point of all experimental parameterizations of windwave spectra (e.g. Hasselmann et al., 1973).

Proceeding with "realistic" wave inputs we, first, justify the effect of strong tendency to self-similar behaviour, and, then, the universality of the spectral shape. Again, we find rather small differences of spectral shapes for different models of wave generation. Quite evidently, this results from dominating nonlinear transfer as compared to wave input and dissipation but the possibility itself of this domination is not evident a priori. The situation looks like a magic circle high non-linearity requires high magnitudes of wave field and these magnitudes, in their turn, require high wave input. Fortunately, nonlinearity is likely a permanent winner in this conflict in a wide range of conditions of wave input and dissipation.

For "realistic" wave inputs the nonlinearity cannot dominate globally, in the whole domain of wave scales and directions. There are frequencies and directions where nonlinearity is relatively small and, hence, does not provide the strong tendency to self-similarity. In this case it is useful to model the wind-wave field as a composite of self-similar "core" and non-self-similar "background". This composite model allows to explain differences of exponents of wave growth observed in experiments and in numerical runs. Spectral peaks are described better by behaviour of self-similar core of solutions while evolution of total energy and mean frequency can be affected essentially by non-self-similar wave background. Taking into account this simple observation we obtain very good coincidence of our numerical results with JONSWAP parameterization of wave spectra. We extract parameter $\kappa_{\alpha}$ of wind-wave growth from numerical solutions. Generally it appears significantly higher than in experiments. The explanation is trivial: we used spectral peak features as a reference for the comparison while experimentalists operate usually by total energy and mean frequency that are affected heavily by non-self-similar wave background.

The final part of the paper brings us back to the fundamental role of the Kolmogorov-Zakharov solutions. We find relationship of the non-stationary (nonhomogeneous) anisotropic self-similar solutions and stationary and isotropic KZ solutions in terms of spectral fluxes. The result looks paradoxical: spectral cascading in both cases is governed by the same set of fundamental physical values $C_{p}$ and $C_{q}$. Former Kolmogorov's constants $C_{p}$ and $C_{q}$ become functions of selfsimilar argument $\xi$ which is nothing but non-dimensional wave scale $\left(\xi=|\boldsymbol{k}| /\left|\boldsymbol{k}_{p}\right|=\omega^{2} / \omega_{p}^{2}\right)$ for the self-similar solutions. These functions characterize ratios of wave spectra magnitudes to spectral fluxes. Our result show that these ratios are independent, or, more carefully, depend weakly on wave input, i.e. the problems studied in this paper show adiabatic development of spectral fluxes.

We presented numerical results for duration-limited growth only. At the moment numerical solution of the fetchlimited problem for the Hasselmann kinetic equation cannot be obtained with accuracy which is adequate to the questions considered above. At the same time, the present study gives a hope that essential features of self-similar evolution of wind wave spectra remain valid in this particular case and, very likely, can facilitate essentially description of spatiotemporal wind-wave growth in general case.

\section{Appendix A Kernels for the kinetic equation}

The four-wave interaction coefficient for gravity waves, satisfying all the symmetry conditions at the resonance curve as well as elsewhere, has the form

$\tilde{V}_{1234}^{(2)}=T_{1234}+\left(\omega_{1}+\omega_{2}-\omega_{3}-\omega_{4}\right) N_{1234}$,

where $T_{1234}$ can be presented in a relatively compact way as

$$
\begin{aligned}
& T_{1234}= \frac{1}{32 \pi^{2}} \frac{1}{\left(q_{1} q_{2} q_{3} q_{4}\right)^{1 / 4}} \\
& \times\left\{\left(-\boldsymbol{k}_{2} \boldsymbol{k}_{3}+q_{2} q_{3}\right)\left(-\boldsymbol{k}_{1} \boldsymbol{k}_{4}+q_{1} q_{4}\right)\right. \\
&+\left(-\boldsymbol{k}_{1} \boldsymbol{k}_{3}+q_{1} q_{3}\right)\left(-\boldsymbol{k}_{2} \boldsymbol{k}_{4}+q_{2} q_{4}\right) \\
&+\left(\boldsymbol{k}_{1} \boldsymbol{k}_{2}+q_{1} q_{2}\right)\left(\boldsymbol{k}_{3} \boldsymbol{k}_{4}+q_{3} q_{4}\right) \\
&+ \omega_{1} \omega_{2} \omega_{3} \omega_{4} \\
& \times\left[q_{1}^{2}+q_{2}^{2}+q_{3}^{2}+q_{4}^{2}-q_{1-3}\left(\omega_{2}-\omega_{4}\right)^{2}\right. \\
& \\
&+ \frac{\left.-q_{2-3}\left(\omega_{2}-\omega_{3}\right)^{2}-q_{1+2}\left(\omega_{3}+\omega_{4}\right)^{2}\right]}{q_{1-3}-\left(\omega_{2}-\omega_{4}\right)^{2}} \\
& \times\left[2 \boldsymbol{k}_{1} \boldsymbol{k}_{3}+\omega_{1} \omega_{3}\left(q_{1}+q_{3}-q_{1-3}\right)\right] \\
& \times\left[2 \boldsymbol{k}_{2} \boldsymbol{k}_{4}+\omega_{2} \omega_{4}\left(q_{2}+q_{4}-q_{1-3}\right)\right] \\
&+ \frac{\left(\omega_{2}-\omega_{3}\right)^{2}}{q_{2-3}-\left(\omega_{2}-\omega_{3}\right)^{2}} \\
& \times\left[2 \boldsymbol{k}_{1} \boldsymbol{k}_{4}+\omega_{1} \omega_{4}\left(q_{1}+q_{4}-q_{2-3}\right)\right] \\
& \times\left[2 \boldsymbol{k}_{2} \boldsymbol{k}_{3}+\omega_{2} \omega_{3}\left(q_{2}+q_{3}-q_{2-3}\right)\right] \\
&+ \frac{\left(\omega_{3}+\omega_{4}\right)^{2}}{q_{1+2}-\left(\omega_{3}+\omega_{4}\right)^{2}} \\
& \times\left[2 \boldsymbol{k}_{1} \boldsymbol{k}_{2}+\omega_{1} \omega_{2}\left(q_{1}+q_{2}-q_{1+2}\right)\right] \\
& \times\left[2 \boldsymbol{k}_{3} \boldsymbol{k}_{4}+\omega_{3} \omega_{4}\left(q_{3}+q_{4}-q_{1+2}\right)\right]
\end{aligned}
$$

Note that:

1. Perhaps it is not the shortest possible form (see below), but it is indeed remarkably symmetric;

2. $g=1$. Otherwize, $g$ would appear in places.

3. Krasitskii's notation is used, in particular $|\boldsymbol{k}|=q$. 
There are two other versions of $T_{1234}$. One, somewhat longer, is suggested by Zakharov (1999). Correcting a number of misprints and omitting $g$, it can be written as

$$
\begin{aligned}
Z_{1234}= & -\frac{1}{32 \pi^{2}} \frac{1}{\left(q_{1} q_{2} q_{3} q_{4}\right)^{1 / 4}}\left\{-12 q_{1} q_{2} q_{3} q_{4}\right. \\
- & 2\left(\omega_{1}+\omega_{2}\right)^{2} \\
& \times\left[\omega_{3} \omega_{4}\left(\boldsymbol{k}_{1} \boldsymbol{k}_{2}-q_{1} q_{2}\right)+\omega_{1} \omega_{2}\left(\boldsymbol{k}_{3} \boldsymbol{k}_{4}-q_{3} q_{4}\right)\right] \\
- & 2\left(\omega_{1}-\omega_{3}\right)^{2} \\
& \times\left[\omega_{2} \omega_{4}\left(\boldsymbol{k}_{1} \boldsymbol{k}_{3}+q_{1} q_{3}\right)+\omega_{1} \omega_{3}\left(\boldsymbol{k}_{2} \boldsymbol{k}_{4}+q_{2} q_{4}\right)\right] \\
- & 2\left(\omega_{1}-\omega_{4}\right)^{2} \\
& \times\left[\omega_{2} \omega_{3}\left(\boldsymbol{k}_{1} \boldsymbol{k}_{4}+q_{1} q_{4}\right)+\omega_{1} \omega_{4}\left(\boldsymbol{k}_{2} \boldsymbol{k}_{3}+q_{2} q_{3}\right)\right] \\
+ & \left(\boldsymbol{k}_{1} \boldsymbol{k}_{2}+q_{1} q_{2}\right)\left(\boldsymbol{k}_{3} \boldsymbol{k}_{4}+q_{3} q_{4}\right) \\
+ & \left(-\boldsymbol{k}_{1} \boldsymbol{k}_{3}+q_{1} q_{3}\right)\left(-\boldsymbol{k}_{2} \boldsymbol{k}_{4}+q_{2} q_{4}\right) \\
+ & \left(-\boldsymbol{k}_{1} \boldsymbol{k}_{4}+q_{1} q_{4}\right)\left(-\boldsymbol{k}_{2} \boldsymbol{k}_{3}+q_{2} q_{3}\right) \\
+ & 4\left(\omega_{1}+\omega_{2}\right)^{2} \frac{\left(\boldsymbol{k}_{1} \boldsymbol{k}_{2}-q_{1} q_{2}\right)\left(\boldsymbol{k}_{3} \boldsymbol{k}_{4}-q_{3} q_{4}\right)}{q_{1+2}-\left(\omega_{1}+\omega_{2}\right)^{2}} \\
+ & 4\left(\omega_{1}-\omega_{3}\right)^{2} \frac{\left(\boldsymbol{k}_{1} \boldsymbol{k}_{3}+q_{1} q_{3}\right)\left(\boldsymbol{k}_{2} \boldsymbol{k}_{4}+q_{2} q_{4}\right)}{q_{1-3}-\left(\omega_{1}-\omega_{3}\right)^{2}} \\
+ & \left.4\left(\omega_{1}-\omega_{4}\right)^{2} \frac{\left(\boldsymbol{k}_{1} \boldsymbol{k}_{4}+q_{1} q_{4}\right)\left(\boldsymbol{k}_{2} \boldsymbol{k}_{3}+q_{2} q_{3}\right)}{q_{2-3}-\left(\omega_{1}-\omega_{4}\right)^{2}}\right\}
\end{aligned}
$$

There exists yet another version of $T_{1234}$ that can be found in the monograph by Lavrenov (2003) and used in his derivation of the specific algorithm for the solution of the Hasselmann equation. Apparently, it is eventually inherited from paper by Webb (1978). Following the above normalization and again omitting $g$, the following formula is obtained:

$$
\begin{aligned}
L_{1234}= & -\frac{1}{32 \pi^{2}} \frac{1}{\left(q_{1} q_{2} q_{3} q_{4}\right)^{1 / 4}}\left\{5 q_{1} q_{2} q_{3} q_{4}\right. \\
+ & 2\left(\omega_{1}+\omega_{2}\right)^{2}\left(\omega_{1}-\omega_{3}\right)^{2}\left(\omega_{1}-\omega_{4}\right)^{2} \\
& \times\left(q_{1}+q_{2}+q_{3}+q_{4}\right) \\
+ & \left(\boldsymbol{k}_{1} \boldsymbol{k}_{2}\right)\left(\boldsymbol{k}_{3} \boldsymbol{k}_{4}\right)+\left(\boldsymbol{k}_{1} \boldsymbol{k}_{3}\right)\left(\boldsymbol{k}_{2} \boldsymbol{k}_{4}\right)+\left(\boldsymbol{k}_{1} \boldsymbol{k}_{4}\right)\left(\boldsymbol{k}_{2} \boldsymbol{k}_{3}\right) \\
- & \frac{1}{2}\left[\left(\omega_{1}+\omega_{2}\right)^{4}\left(\boldsymbol{k}_{1} \boldsymbol{k}_{2}+\boldsymbol{k}_{3} \boldsymbol{k}_{4}\right)\right. \\
& -\left(\omega_{1}-\omega_{3}\right)^{4}\left(\boldsymbol{k}_{1} \boldsymbol{k}_{3}+\boldsymbol{k}_{2} \boldsymbol{k}_{4}\right) \\
& \left.-\left(\omega_{1}-\omega_{4}\right)^{4}\left(\boldsymbol{k}_{1} \boldsymbol{k}_{4}+\boldsymbol{k}_{2} \boldsymbol{k}_{3}\right)\right] \\
+ & \frac{4\left(\omega_{1}-\omega_{3}\right)^{2}\left(\boldsymbol{k}_{1} \boldsymbol{k}_{3}+q_{1} q_{3}\right)\left(\boldsymbol{k}_{2} \boldsymbol{k}_{4}+q_{2} q_{4}\right)}{q_{1-3}-\left(\omega_{1}-\omega_{3}\right)^{2}} \\
+ & \frac{4\left(\omega_{1}-\omega_{4}\right)^{2}\left(\boldsymbol{k}_{1} \boldsymbol{k}_{4}+q_{1} q_{4}\right)\left(\boldsymbol{k}_{2} \boldsymbol{k}_{3}+q_{2} q_{3}\right)}{q_{2-3}-\left(\omega_{1}-\omega_{4}\right)^{2}} \\
+ & \frac{4\left(\omega_{1}+\omega_{2}\right)^{2}\left(q_{1} q_{2}-\boldsymbol{k}_{1} \boldsymbol{k}_{2}\right)\left(q_{3} q_{4}-\boldsymbol{k}_{3} \boldsymbol{k}_{4}\right)}{q_{1+2}-\left(\omega_{1}+\omega_{2}\right)^{2}}
\end{aligned}
$$

Though $T, Z$ and $L$ look quite differently, they are all numerically equivalent at the resonance curve. However, it is important to note that they are not equivalent out of it, i.e. they differ on certain functions proportional to $\left(\omega_{1}+\omega_{2}-\omega_{3}-\omega_{4}\right)$.

The expression for $N_{1234}$ depends on the specific choice of the canonical transformation. Using the transformation of Krasitskii, the following expression (unfortunately, rather long) can be obtained (certainly, it is valid for $T_{1234}$ only, and not valid for $Z$ and $L$ ):

$$
\begin{aligned}
& N_{1234}=\frac{1}{32 \pi^{2}} \frac{1}{\left(q_{1} q_{2} q_{3} q_{4}\right)^{1 / 4}} \\
& \times\left\{\omega _ { 1 } \omega _ { 2 } \omega _ { 3 } \omega _ { 4 } \left[q_{1} \omega_{1}+q_{2} \omega_{2}-q_{3} \omega_{3}-q_{4} \omega_{4}\right.\right. \\
& -q_{1-3}\left(\omega_{2}-\omega_{4}\right)-q_{2-3}\left(\omega_{2}-\omega_{3}\right) \\
& \left.+q_{1+2}\left(\omega_{3}+\omega_{4}\right)\right] \\
& +\frac{\left(\omega_{2}-\omega_{3}\right)}{q_{2-3}-\left(\omega_{2}-\omega_{3}\right)^{2}} \\
& \times\left[\boldsymbol{k}_{1} \boldsymbol{k}_{4}+q_{1} q_{4}+\omega_{1} \omega_{4}\left(q_{1}+q_{4}-q_{2-3}\right)\right] \\
& \times\left[2 \boldsymbol{k}_{2} \boldsymbol{k}_{3}+\omega_{2} \omega_{3}\left(q_{2}+q_{3}-q_{2-3}\right)\right] \\
& +\frac{\left(\omega_{2}-\omega_{4}\right)}{q_{1-3}-\left(\omega_{2}-\omega_{4}\right)^{2}} \\
& \times\left[\boldsymbol{k}_{1} \boldsymbol{k}_{3}+q_{1} q_{3}+\omega_{1} \omega_{3}\left(q_{1}+q_{3}-q_{1-3}\right)\right] \\
& \times\left[2 \boldsymbol{k}_{2} \boldsymbol{k}_{4}+\omega_{2} \omega_{4}\left(q_{2}+q_{4}-q_{1-3}\right)\right] \\
& -\frac{\left(\omega_{3}+\omega_{4}\right)}{q_{1+2}-\left(\omega_{3}+\omega_{4}\right)^{2}} \\
& \times\left[-\boldsymbol{k}_{1} \boldsymbol{k}_{2}+q_{1} q_{2}-\omega_{1} \omega_{2}\left(q_{1}+q_{2}-q_{1+2}\right)\right] \\
& \times\left[-2 \boldsymbol{k}_{3} \boldsymbol{k}_{4}-\omega_{3} \omega_{4}\left(q_{3}+q_{4}-q_{1+2}\right)\right] \\
& +\left[\omega_{2-3}\left(q_{2} q_{3}-\boldsymbol{k}_{2} \boldsymbol{k}_{3}\right)\right. \\
& -\omega_{2}\left(\boldsymbol{k}_{3} \boldsymbol{k}_{2-3}+q_{3} q_{2-3}\right) \\
& \left.+\omega_{3}\left(q_{2} q_{2-3}-\boldsymbol{k}_{2} \boldsymbol{k}_{2-3}\right)\right] \\
& \times\left[\omega_{2-3}\left(q_{1} q_{4}-\boldsymbol{k}_{1} \boldsymbol{k}_{4}\right)\right. \\
& +\omega_{1}\left(q_{4} q_{2-3}-\boldsymbol{k}_{4} \boldsymbol{k}_{2-3}\right) \\
& \left.-\omega_{4}\left(\boldsymbol{k}_{1} \boldsymbol{k}_{2-3}+q_{1} q_{2-3}\right)\right] \\
& \times\left[4 \omega_{2-3}\left(\omega_{2-3}+\omega_{1}-\omega_{4}\right)\left(\omega_{2-3}-\omega_{2}+\omega_{3}\right)\right]^{-1} \\
& -\left[\omega_{2-3}\left(-\boldsymbol{k}_{2} \boldsymbol{k}_{3}+q_{2} q_{3}\right)\right. \\
& +\omega_{2}\left(\boldsymbol{k}_{3} \boldsymbol{k}_{2-3}+q_{3} q_{2-3}\right) \\
& \left.-\omega_{3}\left(-\boldsymbol{k}_{2} \boldsymbol{k}_{2-3}+q_{2} q_{2-3}\right)\right] \\
& \times\left[\omega_{2-3}\left(-\boldsymbol{k}_{1} \boldsymbol{k}_{4}+q_{1} q_{4}\right)\right. \\
& -\omega_{1}\left(-\boldsymbol{k}_{4} \boldsymbol{k}_{2-3}+q_{4} q_{2-3}\right) \\
& \left.+\omega_{4}\left(\boldsymbol{k}_{1} \boldsymbol{k}_{2-3}+q_{1} q_{2-3}\right)\right] \\
& \times\left[4 \omega_{2-3}\left(\omega_{2-3}-\omega_{1}+\omega_{4}\right)\left(\omega_{2-3}+\omega_{2}-\omega_{3}\right)\right]^{-1} \\
& +\left[\omega_{1-3}\left(-\boldsymbol{k}_{2} \boldsymbol{k}_{4}+q_{2} q_{4}\right)\right. \\
& -\omega_{2}\left(\boldsymbol{k}_{4} \boldsymbol{k}_{1-3}+q_{4} q_{1-3}\right) \\
& \left.+\omega_{4}\left(-\boldsymbol{k}_{2} \boldsymbol{k}_{1-3}+q_{2} q_{1-3}\right)\right] \\
& \times\left[\omega_{1-3}\left(-\boldsymbol{k}_{1} \boldsymbol{k}_{3}+q_{1} q_{3}\right)\right. \\
& +\omega_{1}\left(-\boldsymbol{k}_{3} \boldsymbol{k}_{1-3}+q_{3} q_{1-3}\right) \\
& \left.-\omega_{3}\left(\boldsymbol{k}_{1} \boldsymbol{k}_{1-3}+q_{1} q_{1-3}\right)\right] \\
& \times\left[4 \omega_{1-3}\left(\omega_{1-3}+\omega_{1}-\omega_{3}\right)\left(\omega_{1-3}-\omega_{2}+\omega_{4}\right)\right]^{-1} \\
& -\left[\omega_{1-3}\left(-\boldsymbol{k}_{2} \boldsymbol{k}_{4}+q_{2} q_{4}\right)\right. \\
& +\omega_{2}\left(\boldsymbol{k}_{4} \boldsymbol{k}_{1-3}+q_{4} q_{1-3}\right) \\
& \left.-\omega_{4}\left(-\boldsymbol{k}_{2} \boldsymbol{k}_{1-3}+q_{2} q_{1-3}\right)\right] \\
& \times\left[\omega_{1-3}\left(-\boldsymbol{k}_{1} \boldsymbol{k}_{3}+q_{1} q_{3}\right)\right.
\end{aligned}
$$




$$
\begin{aligned}
& -\omega_{1}\left(-\boldsymbol{k}_{3} \boldsymbol{k}_{1-3}+q_{3} q_{1-3}\right) \\
& \left.+\omega_{3}\left(\boldsymbol{k}_{1} \boldsymbol{k}_{1-3}+q_{1} q_{1-3}\right)\right] \\
& {\left[4 \omega_{1-3}\left(\omega_{1-3}-\omega_{1}+\omega_{3}\right)\left(\omega_{1-3}+\omega_{2}-\omega_{4}\right)\right]^{-1} } \\
+ & {\left[\omega_{1+2}\left(\boldsymbol{k}_{1} \boldsymbol{k}_{2}+q_{1} q_{2}\right)\right.} \\
& +\omega_{2}\left(-\boldsymbol{k}_{1} \boldsymbol{k}_{1+2}+q_{1} q_{1+2}\right) \\
& \left.+\omega_{1}\left(-\boldsymbol{k}_{2} \boldsymbol{k}_{1+2}+q_{2} q_{1+2}\right)\right] \\
& \times\left[\omega_{1+2}\left(\boldsymbol{k}_{3} \boldsymbol{k}_{4}+q_{3} q_{4}\right)\right. \\
& +\omega_{4}\left(-\boldsymbol{k}_{3} \boldsymbol{k}_{1+2}+q_{3} q_{1+2}\right) \\
& \left.+\omega_{3}\left(-\boldsymbol{k}_{4} \boldsymbol{k}_{1+2}+q_{4} q_{1+2}\right)\right] \\
& \times\left[4 \omega_{1+2}\left(\omega_{1+2}+\omega_{1}+\omega_{2}\right)\left(\omega_{1+2}+\omega_{3}+\omega_{4}\right)\right]^{-1} \\
- & {\left[\omega_{1+2}\left(\boldsymbol{k}_{1} \boldsymbol{k}_{2}+q_{1} q_{2}\right)\right.} \\
& -\omega_{2}\left(-\boldsymbol{k}_{1} \boldsymbol{k}_{1+2}+q_{1} q_{1+2}\right) \\
& \left.-\omega_{1}\left(-\boldsymbol{k}_{2} \boldsymbol{k}_{1+2}+q_{2} q_{1+2}\right)\right] \\
& \times\left[\omega_{1+2}\left(\boldsymbol{k}_{3} \boldsymbol{k}_{4}+q_{3} q_{4}\right)\right. \\
& -\omega_{4}\left(-\boldsymbol{k}_{3} \boldsymbol{k}_{1+2}+q_{3} q_{1+2}\right) \\
& \left.-\omega_{3}\left(-\boldsymbol{k}_{4} \boldsymbol{k}_{1+2}+q_{4} q_{1+2}\right)\right] \\
& \times\left[4 \omega_{1+2}\left(\omega_{1+2}-\omega_{1}-\omega_{2}\right)\left(\omega_{1+2}-\omega_{3}-\omega_{4}\right)\right]^{-1}
\end{aligned}
$$

The expression $T_{1234}+\left(\omega_{1}+\omega_{2}-\omega_{3}-\omega_{4}\right) N_{1234}$ coincides with Krasitskii's $\tilde{V}_{1234}^{(2)}$ globally.

\section{Appendix B Green function for function $A(\omega, \theta)$}

Solution for the equation

$$
L A=\left(\frac{1}{2} \frac{\partial^{2}}{\partial \omega^{2}}+\frac{1}{\omega^{2}} \frac{\partial^{2}}{\partial \theta^{2}}\right) A=f(\omega, \theta)
$$

is given by Green function

$$
A(\omega, \theta)=\int_{0}^{\infty} \int_{0}^{2 \pi} G\left(\omega, \omega^{\prime}, \theta-\theta^{\prime}\right) f\left(\omega, \omega^{\prime}\right) d \omega^{\prime} d \theta^{\prime}
$$

where

$$
\begin{array}{r}
G\left(\omega, \omega^{\prime}, \theta-\theta^{\prime}\right)=-\frac{1}{2 \pi} \sum_{n=-\infty}^{\infty} \frac{\sqrt{\omega \omega^{\prime}}}{\Delta_{n}} \exp \left[\operatorname{in}\left(\theta-\theta^{\prime}\right)\right] \\
\times\left[\left(\frac{\omega^{\prime}}{\omega}\right)^{\Delta_{n}} \theta\left(1-\frac{\omega^{\prime}}{\omega}\right)+\left(\frac{\omega}{\omega^{\prime}}\right)^{\Delta_{n}} \theta\left(\frac{\omega^{\prime}}{\omega}-1\right)\right]
\end{array}
$$

Here

$$
\Delta_{n}=\sqrt{\frac{1}{4}+2 n^{2}}
$$

and

$\theta(\xi)=\left\{\begin{array}{lll}1 & \xi>0 \\ 0 & \xi<0\end{array}\right\}$

Equation (66) appears after substituting of $S_{n l}\left(\omega^{\prime}, \theta^{\prime}\right)$ as $f\left(\omega^{\prime}, \theta^{\prime}\right)$ in Eqs. (63) and (62).

\section{Appendix C Formal solution for function $A(\omega, \theta)$}

To determine the equation, describing the general Kolmogorov solution one defines the following function:

$$
\begin{aligned}
& F(\omega, \theta)=4 \pi \int_{0}^{\infty} d \omega_{1} \int_{0}^{\omega_{3}} d \omega_{2} \int_{\omega}^{\infty} d \omega_{3} \int_{0}^{2 \pi} d \theta_{1} \int_{0}^{2 \pi} d \theta_{2} \int_{0}^{2 \pi} d \theta_{3} \\
& \times \delta\left(\omega+\omega_{1}-\omega_{2}-\omega_{3}\right) \\
& \times \delta\left(\omega \cos \theta+\omega_{1} \cos \theta_{1}-\omega_{2} \cos \theta_{2}-\omega_{3} \cos \theta_{3}\right) \\
& \times\left[\omega^{3} N_{\omega_{1}} N_{\omega_{2}} N_{\omega_{3}}+\omega_{1}^{3} N_{\omega} N_{\omega_{2}} N_{\omega_{3}}\right. \\
& \left.-\omega_{2}^{3} N_{\omega} N_{\omega_{1}} N_{\omega_{3}}-\omega_{3}^{3} N_{\omega} N_{\omega_{1}} N_{\omega_{2}}\right] \cdot\left|T_{\omega \omega_{1} \omega_{2} \omega_{3}, \theta \theta_{1} \theta_{2} \theta_{3}}\right|^{2}
\end{aligned}
$$

and find its Fourier coefficients

$$
F_{n}(\omega)=\int_{0}^{2 \pi} F_{n}(\omega, \theta) \cos n \theta d \theta
$$

A general Kolmogorov spectrum is defined by the following system of equations:

$$
\begin{aligned}
P+\omega Q & =\int_{0}^{\omega}\left(\omega-\omega_{1}\right) F_{0}\left(\omega_{1}\right) d \omega_{1} \\
M & =\frac{1}{g} \int_{0}^{\omega} \omega_{1}^{2} F_{1}\left(\omega_{1}\right) d \omega_{1} \\
F_{n}(\omega) & =0 \quad \text { if } \quad n \geq 2
\end{aligned}
$$

Now $\varepsilon_{\omega}(\theta)=\omega N_{\omega}(\theta)$. One can present $N$ in a form of the Fourier series

$N(\omega, \theta)=\frac{1}{2 \pi} \sum N_{n}(\omega) \cos n \theta$

and turn Eqs. (C3)-(C5) into an infinite system of nonlinear integral equations imposed on $N_{n}(\omega)$.

Acknowledgements. Authors are grateful to S. Annenkov for granting permission to publish his results on different forms of four-wave interaction coefficients for deep water waves (Appendix A). The kernels of the kinetic equation used in our numerical codes satisfy all the formulas presented in Appendix A. The research presented in this paper was conducted under the US Army Corps of Engineers, RDT\&E program, grant DACA 42-00-C0044, ONR grant N00014-98-1-0070 and NSF grant NDMS0072803, INTAS grant 01-234, Russian Foundation for Basic Research N02-05-65140, N04-05-64784, ofi-a-05-05-08027, grant "Russian top-level research schools" NS-1716.2003.1 and Russian Academy Program "Mathematical methods of nonlinear dynamics". This support is gratefully acknowledged.

Edited by: V. I. Shrira

Reviewed by: M. Onorato and another referee 


\section{References}

Annenkov, S. Y. and Shrira, V. I.: Direct numerical simulation of evolution of random water wave fields: direct and inverse cascades, Proceedings of International Symposium Topical Problems of Nonlinear Wave Physics, St. Peterburg-Nizhny Novgorod, 2-9 August 2005, 8-9, 2005.

Annenkov, S. Y. and Shrira, V. I.: Direct numerical simulation of the statistical characteristics of wave ensembles, Doklady Russ. Acad. Sciences, 396, 1-4, 2004a.

Babanin, A. N. and Soloviev, Yu. P.: Field investigation of transformation of the wind wave frequency spectrum with fetch and the stage of development, J. Phys. Oceanogr., 28, 563-576, 1998.

Badulin, S., Pushkarev, A. N., Resio, D., and Zakharov, V.: Direct and inverse cascade of energy, momentum and wave action in wind-driven sea, 7th International workshop on wave hindcasting and forecasting, Banff, October 2002, 92-103, 2002.

Badulin, S. I., Shrira, V. I., Kharif, C., and Ioualalen, M.: On two approaches to the problem of instability of short-crested water waves, J. Fluid Mech. 303, 297-325, 1995.

Balk ,A. M. and Zakharov, V. E.: Stability of weak-turbulent Kolmogorov spectra, Amer. Math. Soc. Transl. Ser. 2, 182, 31-81, 1998.

Balk, A. M. and Zakharov, V. E.: Stability of weakly turbulent Kolmogorov spectra, Sov. Phys. Dokl., 33, 270-272, 1988.

Barrenblatt, G. I.: Scaling, self-similarity, and intermediate asymptotics: Dimensional analysis and intermediate asymptotics, Plenum Press, New York/London, 218 p., 1979.

Battjes, J. A., Zitman, T. J., and Holthuijsen, L. H.: A reanalysis of the spectra observed in JONSWAP, J. Phys. Oceanogr., 17, 1288-1295, 1987.

Benoit, M.: Evaluation of methods to compute the non-linear quadruplet interactions for deep-water wave spectra, Proc. 5th Int. Symp. on Ocean Wave Measurement and Analysis (WAVES'2005), 3-7 July 2005, Madrid (Spain), Paper 52, 10 p., 2005.

Booij, N., Ris, R. C., and Holthuijsen, L. H.: A third-generation wave model for coastal regions, Part I, Model description and validation, J. Geoph. Res., C4, 104, 7649-7666, 1999.

Donelan, M. A., Hamilton, J., and Hui, W. H.: Directional spectra of wind-generated waves, Phil. Trans. R. Soc. Lond., A315, 509562,1985 .

Donelan, M. A. and Pierson-jr., W. J.: Radar scattering and equilibrium ranges in wind-generated waves with application to scatterometry, J. Geoph. Res., 92, 4971-5029, 1987.

Dyachenko, A. I., Korotkevich, A. O., and Zakharov, V. E.: Weak turbulent Kolmogorov spectrum for surface gravity waves, Phys. Rev. Lett., 92(13), 134501, 1-4, 2004.

Glazman, R.: Surface gravity waves at equilibrium with a steady wind, J. Geoph.Res., 99(C3),5249-5262, 1994.

Hara, T. and Belcher, S. E.: Wind forcing in the equilibrium range of wind-wave spectra, J. Fluid Mech., 470, 223-245, 2002.

Hashimoto, N., Haagsma, I. J. G., and Holthuijsen, L. H.: Fourwave interactions in SWAN, Proc. 28th Int. Conf. Coastal Engng, 2003.

Hasselmann, K.: On the nonlinear energy transfer in a gravity wave spectrum, Part 1, J. Fluid Mech., 12, 481-500, 1962.

Hasselmann, K.: On the nonlinear energy transfer in a gravity wave spectrum, Parts 2 and 3, J. Fluid Mech., 15, 273-281 and 385398, 1963.

Hasselmann, K.: On the spectral dissipation of ocean waves due to white capping, Boundary Layer Met., 6, 107-127, 1974.
Hasselmann, K., Barnett, T.P., Bouws, E., Carlson, H., Cartwright, D. E., Enke, K., Ewing, J. A., Gienapp, H., Hasselmann, D. E., Kruseman, P., Meerburg, A., Muller, P., Olbers, D. J., Richter, K., Sell W., and Walden, H.: Measurements of wind-wave growth and swell decay during the Joint North Sea Wave Project (JONSWAP), Dtsch. Hydrogh. Z. Suppl., 12, A8, 1973.

Hasselmann, S., Hasselmann, K. Allender, J. H., and Barnett, T. P.: Computations and parametrizations of the nonlinear energy transfer in a gravity-wave spectrum, Part II, Parameterizations of the nonlinear energy transfer for application in wave models, J. Phys. Oceanogr., 15, 1378-1391, 1985.

Hasselmann, S. and Hasselmann, K.: A symmetrical method of computing the nonlinear transfer in a gravity wave spectrum Hamburger Geophysikalische Einzelschriften, Reihe A, Heft 52, Max-Planck-Institut für Meteorologie, Hamburg, 1981.

Hasselmann, S. and Hasselmann, K.: Computations and parametrizations of the nonlinear energy transfer in a gravitywave spectrum, Part I, A new method for efficient computations of the exact nonlinear transfer integral, J. Phys. Oceanogr., 15, 1369-1377, 1985.

Hsiao, S. V. and Shemdin, O. H.: Measurements of wind velocity and pressure with a wave follower during MARSEN, J. Geoph. Res., 88, C14, 9841-9849, 1983.

Iroshnikov, R.: Possibility of formation of a nonisotropic spectrum of wind waves by their weak nonlinear interaction, Soviet Physics Doklady, 280, 1321-1325, 1986.

Janssen P. A. E. M.: Nonlinear four-wave interactions and freak waves, J. Phys. Oceanogr., 33, 863-884, 2003.

Kahma, K. K.: A study of the growth of the wave spectrum with fetch, J. Phys. Oceanogr., 11, 1505-1515, 1981.

Katz, A. V. and Kontorovich, V. M.: Drift stationary solutions in the weak turbulence theory, JETP Lett., 14, 265-267, 1971.

Katz, A. V. and Kontorovich, V. M.: Anisotropic turbulent distributions for waves with a non-decay dispersion law, Soviet Physics JETP, 38, 102-107, 1974.

Katz, A. V. and Kontorovich, V. M., Moiseev S. S., and Novikov V. E.: Power-like solutions of the kinetic Boltzmann equation for distributions of particles with spectral fluxes, JETP Lett., 21, 5-6, 1975.

Kats, A. V., Kantorovich, V. M., Moiseev, S. S., and Novikov, V. E: Exact power law solutions of the particle kinetic equations, Soviet Physics JETP, 44, 93-103, 1976.

Kitaigorodskii, S. A.: Applications of the theory of similarity to the analysis of wind-generated wave motion as a stochastic process, Bull. Acad. Sci. USSR, Geophys. Ser., Engl. Transl., N1, 105117, 1962.

Krasitskii, V. P.: On reduced Hamiltonian equations in the nonlinear theory of water surface waves, J. Fluid Mech., 272, 1-20, 1994.

Kraichnan, R. H.: Inertial ranges in two-dimensional turbulence, Phys. Fluids, 10, 1417-1423, 1967.

Komatsu, K. and Masuda, A.: A new scheme of nonlinear energy transfer among wind waves: RIAM method, Algorithm and perfomance, J. Oceanogr., 52, 509-537, 1996.

Komen, G. J., Hasselmann, S., and Hasselmann, K.: On the existence of a fully developed wind-sea spectrum, J. Phys. Oceanogr., 14, 1271-1285, 1984.

Komen G. J., Cavaleri, L., Donelan, M., Hasselmann, K., Hasselmann, S., and Janssenn, P. A. E. M.: Dynamics and modelling of ocean waves, Cambridge University Press, 532 pp., 1995.

Lavrenov, I. V.: Wind waves in ocean, Physics and numerical simulation, Springer-Verlag, 386 p., 2003.

Lavrenov, I., Resio, D., and Zakharov, V.: Numerical simulation 
of weak turbulent Kolmogorov spectrum in water surface waves, 7th International workshop on wave hindcasting and forecasting, Banff, October 2002, 104-116, 2002.

Lavrova, O. Yu.: On transversal instability of finite depth water waves, Izv. Atm. Ocean. Phys., 19, 1068-1074, 1983.

Longuet-Higgins, M. S.: Parasitic capillary waves: a direct calculation, J. Fluid Mech., 301, 79-107, 1995.

Longuet-Higgins, M. S.: Progress towards understanding how waves break. Invited lecture, The 21st Symposium on Naval Hydrodynamics, 7-28, 1996.

Masuda, A.: Nonlinear energy transfer between wind waves, J. Phys. Oceanogr., 10, 2082-2092, 1980.

Masuda, A.: Nonlinear energy transfer between random gravity waves, in: Waves Dynamics and Radio Probing of the Ocean Surface, edited by: Phillips, O. M. and Hasselmann, K., Plenum Press, New York, 136-149, 1986.

Melville, W. K.: The role of surface-wave breaking in air-sea interaction, Annual Review of Fluid Mechanics, 28, 279-321, 1996.

Nordheim, L. W.: On the kinetic method in the new statistics and its applications in the electron theory of conductivity, Proc. Roy. Soc. London, A, 119, 689-698, 1928.

Onorato, M., Osborne, A., Serio, M., Resio, D., Pushkarev, A., Brandini, C., and Zakharov, V. E.: Freely Decaying Weak Turbulence for Sea Surface Gravity Waves, Phys. Rev. Lett. 89, 144501, 1-3, 2002.

Onorato, M., Osborne, A., Serio, M., and Cavaleri L.: QuasiResonant Interactions and Non-gaussian statistics in long crested waves, Proceedings of Rogue waves 2004, Brest, 20-22 October, 2004.

Onorato, M., Osborne, A., and Serio, M.: On deviations from Guassian statistics for surface gravity waves, 14th 'Aha Huliko'a Winter Workshop ROGUE WAVES, 24-28 January, 79-83, 2005.

Phillips, O. M.: The equilibrium range in the spectrum of windgenerated waves, J. Fluid Mech., 4, 426-434, 1958.

Phillips, O. M.: On the dynamics of unsteady gravity waves of finite amplitude J. Fluid Mech. 9, 193-217, 1960.

Phillips, O. M.: Spectral and statistical properties of the equilibrium range in wind-generated gravity waves, J.Fluid Mech. 156, 505531, 1985.

Pierson, W. J. and Moskowitz, L. A.: A proposed spectral form for fully developed wind seas based on the similarity theory of S. A. Kitaigorodsii, J. Geophys. Res. 69, 5181-5190, 1964.

Plant W. J.: A relationship between wind stress and wave slope, J. Geoph. Res., 87, C3, 1961-1967, 1982.

Polnikov, V. G.: Numerical Solution of the kinetic equation for surface gravity waves, Izv. Akad. Nauk SSSR, Fiz. Atmos. Okeana, 26, 2, 168-176, 1990.

Polnikov, V. G.: Numerical Modeling of the formation of flux spectra for surface gravity waves, Izv. Akad. Nauk, Fiz. Atmos. Okeana, 29, 6, 837-841, 1993.

Pushkarev, A. N., Resio, D., and Zakharov, V. E.: Second generation diffusion model of interacting gravity waves on the surface of deep fluid, Nonlin. Processes Geophys., 11, 329-342, 2004,

\section{SRef-ID: 1607-7946/npg/2004-11-329.}

Pushkarev, A. N., Resio, D., and Zakharov, V. E.: Weak turbulent theory of the wind-generated gravity sea waves, Physica D: Nonlinear Phenomena, 184, 29-63, 2003.

Resio, D. T. and Perrie, W. A.: Implications of an $f^{-4}$ equilibrium range for wind-generated waves, J. Phys. Oceanogr., 19, 193204, 1989.

Resio, D. and Perrie, W.: A numerical study of nonlinear energy fluxes due to wave-wind interactions, J. Fluid. Mech., 223, 603-
629, 1991.

Snyder, R. L., Dobson, F. W., Elliot, J. A., and Long, R. B.: Array measurements of atmospheric pressure fluctuations above surface gravity waves, J. Fluid. Mech., 102, 1-59, 1981.

Stewart, R. W.: The air-sea momentum exchange, Boundary-Layer Meteorology, 6, 151-167, 1974.

Rasmussen, J. H. and Stiassnie, M.: Discretization of Zakharov's equation, J. Fluid. Mech., 6, 151-167, 1999.

Tanaka, M.: A method of studying nonlinear random field of surface gravity waves by direct numerical simulation, Fluid Dyn. Res. 28, 41-60, 2000.

Toba, Y.: Local balance in the air-sea boundary processes, III. On the spectrum of wind waves, J. Oceanogr. Soc. Japan, 29, 209220, 1973.

Toba, Y.: Wind-wave strong wave interactions and quasi-local equilibrium between wind and wind sea with the friction velocity proportionality, in: Nonlinear ocean waves, edited by: Perrie, W., Advances in Fluid Mechanics, 17, 1-59, 1997.

Tolman, H. J.: Effects of numerics on the physics in a thirdgeneration wind-wave model, J. Phys. Oceanogr., 22, 10951111, 1992.

Tolman, H. L. and Chalikov, D.: Source terms in a third-generation wind wave model, J. Phys. Ocean., 26, 2497-2518, 1996.

Tracy, B. and Resio, D. T.: Theory and calculation of the nonlinear energy transfer between sea waves in deep water, WES Rep. 11, US Army, Engineer Waterways Experiment Station, Vicksburg, MS, 1982.

Webb, D. J.: Non-linear transfers between sea waves, Deep-Sea Res., 25, 279-298, 1978.

Young, I. R.: Wind Generated Ocean Waves, Elsevier, 306 pp., 1999.

Zakharov, V. E.: Problems of the theory of nonlinear surface waves, $\mathrm{PhD}$ thesis, Budker Institute for Nuclear Physics, Novosibirsk, USSR, 1966.

Zakharov, V. E.: Stability of periodic waves of finite amplitude on the surface of a deep fluid, J. Appl. Mech. Tech. Phys. (USSR), 9, 86-94, 1968.

Zakharov, V. E.: Statistical theory of gravity and capillary waves on the surface of a finite-depth fluid, Eur. J. Mech. B/Fluids, 18, 327-344, 1999.

Zakharov, V. E.: Theoretical interpretation of fetch limited winddriven sea observations, 7th International workshop on wave hindcasting and forecasting, Banff, October 2002, 86-92, 2002.

Zakharov, V. E.: Direct and inverse cascades in the wind-driven sea, AGU Geophysical Monograph, Miami, 1-9, 2005.

Zakharov, V. E. and Filonenko, N. N.: Energy spectrum for stochastic oscillations of the surface of a fluid, Dokl. Acad. Nauk USSR, 160, 1292-1295, 1966.

Zakharov, V. E. and Zaslavsky, M. M.: The kinetic equation and Kolmogorov spectra in the weak-turbulence theory of wind waves, Izv. Atm. Ocean. Phys., 18, 747-753, 1982.

Zakharov, V. E. and Zaslavsky, M. M.: Dependence of wave parameters on the wind velocity, duration of its action and fetch in the weak-turbulence theory of water waves, Izv. Atm. Ocean. Physics, 19, 300-306, 1983.

Zakharov, V. E. and Pushkarev, A. N.: Diffusion model of interacting gravity waves on the surface of deep fluid, Nonlin. Processes Geophys., 6, 1-10, 1999,

SRef-ID: 1607-7946/npg/1999-6-1.

Zakharov, V. E., Korotkevich, A. O., Pushkarev, A. N., and Dyachenko, A. I.: Mesoscopic wave turbulence, JETP Lett., 82, 8, 544-548, 2005. 